\title{
Determination of vitality from a non-invasive biomedical measurement for use in integrated biometric devices
}

\author{
Reza Derakhshani \\ West Virginia University
}

Follow this and additional works at: https://researchrepository.wvu.edu/etd

\section{Recommended Citation}

Derakhshani, Reza, "Determination of vitality from a non-invasive biomedical measurement for use in integrated biometric devices" (1999). Graduate Theses, Dissertations, and Problem Reports. 1522. https://researchrepository.wvu.edu/etd/1522

This Thesis is protected by copyright and/or related rights. It has been brought to you by the The Research Repository @ WVU with permission from the rights-holder(s). You are free to use this Thesis in any way that is permitted by the copyright and related rights legislation that applies to your use. For other uses you must obtain permission from the rights-holder(s) directly, unless additional rights are indicated by a Creative Commons license in the record and/ or on the work itself. This Thesis has been accepted for inclusion in WVU Graduate Theses, Dissertations, and Problem Reports collection by an authorized administrator of The Research Repository @ WVU. For more information, please contact researchrepository@mail.wvu.edu. 


\title{
D etermination of Vitality From A N on-Invasive Biomedical M easurement for U se in Integrated Biometric D evices
}

\author{
Reza D erakhshani, B.S.E.E.
}

\section{THESIS}

Submitted to

The Department of Computer Science and Electrical Engineering of the College of Engineering and Mineral Resources at WEST VIRGINIA UNIVERSITY in partial fulfillment of the requirements for the degree of Master of Science in Electrical Engineering

Committee:

Stephanie Schuckers, Ph.D., Chair Lawrence Hornak, Ph.D. Powsiri Klinkhachorn, Ph.D.

Morgantown, West Virginia, 1999

Keywords: Fingerprints, Vitality, Biometrics, Non-invasive methods, Neural networks, Capacitive scanners, Image processing Copyright 1999 Reza Derakhshani 


\section{ABSTRACT}

\section{Determination of Vitality from A N on-Invasive Biomedical M easurement for U se in Integrated Biometric D evices}

\section{Reza D erakhshani}

Personal identification is a very important issue in today's complex, mobile and electronically networked societies. Among the available measures, fingerprints are the oldest and most widely used. Unfortunately, depending on the capturing technique, it is usually possible to deceive automatic fingerprint identification systems by presenting a well-duplicated synthetic or dismembered finger. This project is one method to provide fingerprint vitality authentication in order to solve the spoof-attack problem. Using a sensor that is composed of an array of capacitors, this method identifies the vitality of a fingerprint by looking at a series of fingerprints captured during a 5-second time frame and detects a perspiration pattern over the human skin. Mapping the two-dimensional images into one-dimensional signals, two ensembles of measures, namely static and dynamic measures, are used for classification. Static patterns as well as temporal changes in dielectric mosaic structure of the skin -caused by perspiration - demonstrate themselves in these signals. Using these measures, this algorithm quantifies the sweating pattern and makes a final decision about vitality of the fingerprint by a neural network trained by examples.

\section{Sponsoring Agency: Veridicom Inc., Santa Clara, CA}




\section{Dedication}

I dedicate this thesis to my family, especially my mother, Shahzad, for her infinite love, help and support through my life and education. 


\section{Acknowledgement}

I wish to thank my advisor, Dr. Stephanie Schuckers, for her endless efforts and invaluable advice. I would like to thank my committee members, Dr. Lawrence Hornak

and Dr. Powsiri Klinkhachorn, for their precious time and help. I would also like to thank all the people in WVU's Health Science Center, especially Nina Clovis and Dr. Mohssen Ghalichebaf, without whose help this research would not be possible. 


\section{Table of Contents}

CHAPTER 1. INTRODUCTION AND BACKGROUND..................................... 1

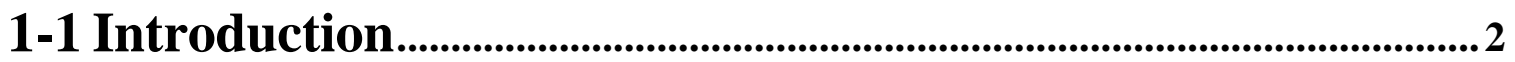

1-1-1 Project Statement ............................................................................. 2

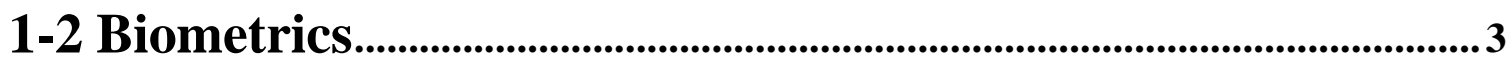

1-3 Applications..........................................................................................

1-4 Fingerprints and Related Fraud ..................................................4

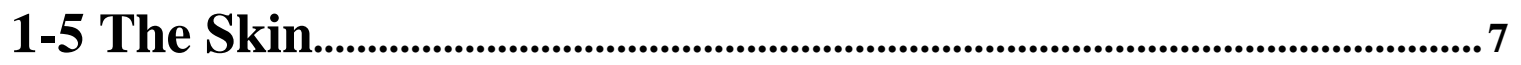

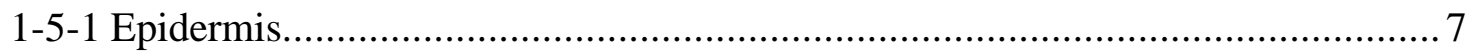

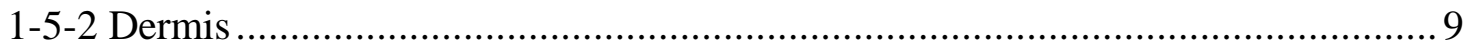

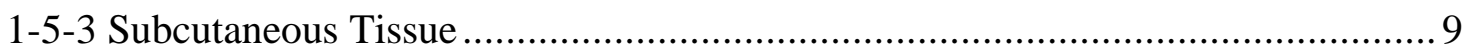

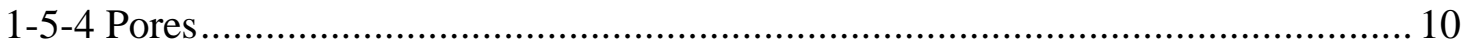

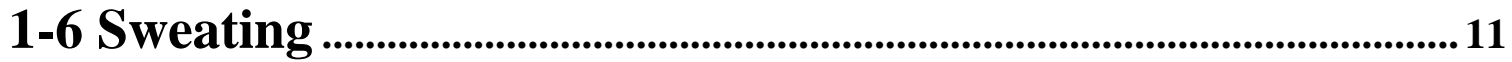

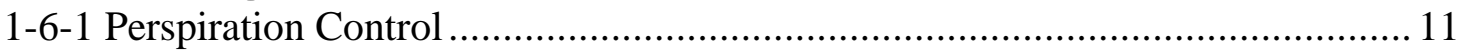

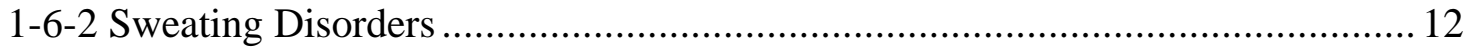

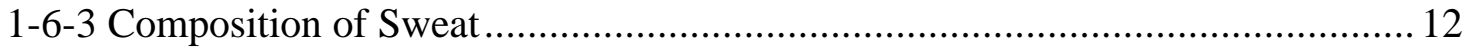

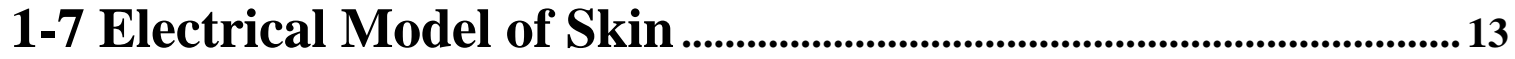

1-8 Fingerprint Scanner ........................................................................ 16

1-8-1 Modeling of the Veridicom FPS 100 Sensor Cell ......................................... 18

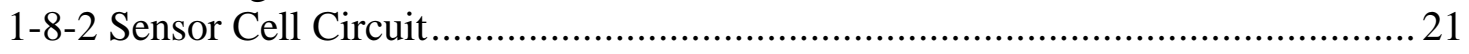

1-9 Artificial Neural Networks................................................................. 25

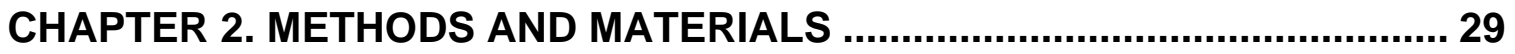

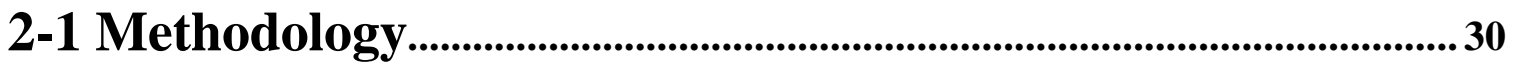

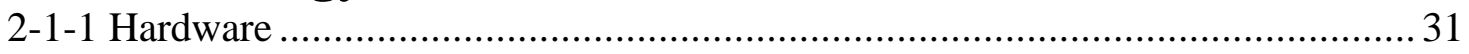


2-1-2 Software

2-1-3 Training and Test Set.

2-1-4 Spoof Development

2-2 Description of Physiologic Phenomenon: 34

2-3 The Algorithm 37

2-3-1 Overview

2-3-2 Detailed Description

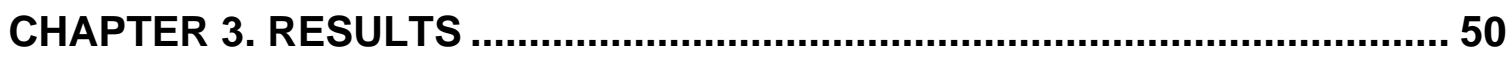

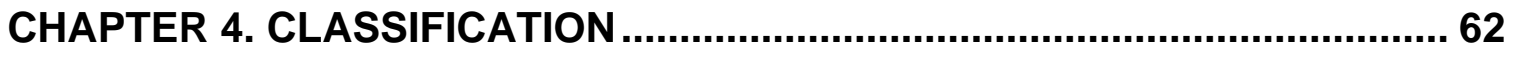

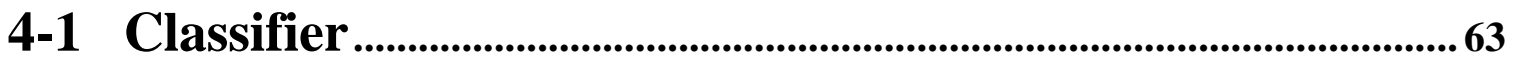

4-2 Neural Networks .................................................................................... 65

CHAPTER 5. CONCLUSION AND FUTURE WORK ........................................ 69

5-1 Conclusion ............................................................................................ 70

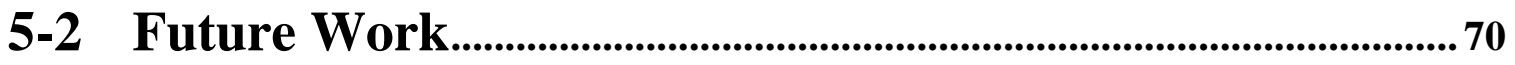

5-3 Contributions and Publications ...................................................... 71

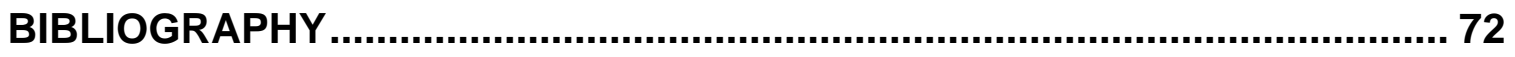

APPENDIX A: SPECIFICATIONS OF FPS100, SOLID STATE FINGERPRINT



APPENDIX B: FINGER FREQUENCY RESPONSE TESTS.......................... 79

TEST SET-UP FOR FINGER FREQUENCY RESPONSE MEASUREMENT.... 80

APPENDIX C: A COMPARISON BETWEEN FRESH AND FROZEN SKIN ..... 85

APPENDIX D: SAMPLE FINGERPRINTS AND RELATED OUTPUTS ............ 87

APPENDIX E: FLOWCHART OF THE ALGORITHM..................................... 124 


\section{Table of Figures}

Figure 1-1 Fingertip skin histology using 80x magnification. ……......................... 8

Figure 1-2 Schematic of general skin structure including various layers and estimated

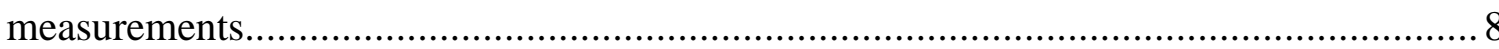

Figure 1-3 Cross section of (the outer layer of) skin as a parallel combination of resistors and capacitors

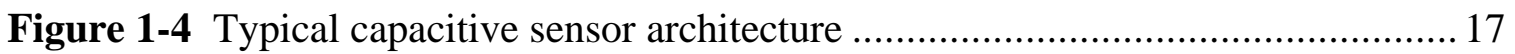

Figure 1-4 Schematic used in calculating capacitance detected by the sensor................ 18

Figure 1-5 Veridicom FPS 100 sensor cell with sample and hold circuit [14]...............21

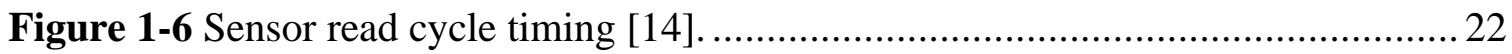

Figure 1-7 A simple neuron, consisting of weighted inputs $w_{i} x_{i}$, a bias $b$, and nonlinear function $\mathbf{f}$.

Figure 2-1 Soaked rubber based spoof (left and center), and play dough spoof (right)... 32

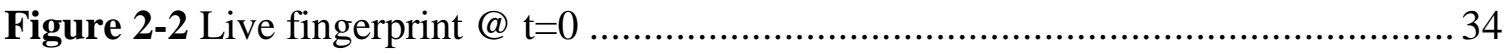

Figure 2-3 Live fingerprint @ t=5 ........................................................................... 34

Figure 2-4 Nine scans (from left to right and top to bottom) collected over 5 seconds... 35

Figure 2-5 The first (left) and last (right) scan of a cadaver fingerprint. ........................ 36

Figure 2-6 The first (left) and last (right) scan of a spoof fingerprint.............................. 36

Figure 2-7 Original scan (left), blank scan (center) and scan after noise and defects removed (right).

Figure 2-8 Image after step 2 (left), step 3 (right)

Figure 2-9 Fingerprint ridges as found by steps 4,5 , and 6 overlaid on the fingerprint image

Figure 2-10 The average of the FFTs calculated from signal segments from the first (top) and last (bottom) capture from the live fingerprint shown in Figure 2-19.

Figure 2-11 Same as above for a fingerprint from a cadaver.

Figure 2-12 Same as above for a spoof fingerprint. 43 


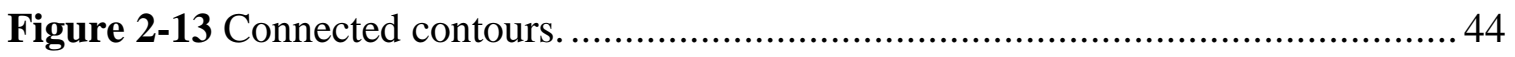

Figure 2-14 Portion of a live fingerprint signal. * denotes minimums and maximums... 45 Figure 2-15 Portion of a cadaver fingerprint signal. * denotes minimums and maximums.

Figure 2-16 Portion of a spoof fingerprint signal, * denotes maximums and minimums.46 Figure 3-1 Static measure (SM). The energy (y-axis) for live (top) and spoof (bottom) corresponding to pore separation of 8-24 pixels $(0.4-1.2 \mathrm{~mm})$, dashed for last capture and solid for first. Note that the vertical scales are different. The X-axis is "sorted" subjects.52 Figure 3-2 ROC associated with Figure 3-1. The line is the FAR and FRR due to threshold change for the static measure. FAR is false acceptance rate of spoof detected as live and FRR is the false rejection rate of live detected as spoof. 52 Figure 3-3 Static measure (SM). The energy (y-axis) for live (top) and cadaver (bottom) corresponding to pore separation of 8-24 pixels $(0.4-1.2 \mathrm{~mm})$, dashed for last capture and solid for first. Note that vertical scales are different.

Figure 3-4 The ROC associated with Figure 3-3. The line is the FAR and FRR due to threshold change for the static measure. FAR is false acceptance rate of cadaver detected as live and FRR is the false rejection rate of live detected as cadaver..........................53

Figure 3-5 First/last total swing ratio (DM1) for live (solid) and spoof (dashed)..........54

Figure 3-6 The ROC associated with Figure 3-5................................................ 54

Figure 3-7 First/last total swing ratio (DM1) for live (solid) and cadaver (dashed)....... 55

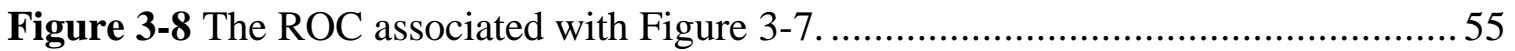

Figure 3-9 Min/max growth ratio (DM2) for live (solid) and spoof (dashed)................56

Figure 3-10 The ROC associated with Figure 3-9.................................................. 56

Figure 3-11 Min/max growth ratio (DM2) for live (solid) and cadaver (dashed)..........57

Figure 3-13 Mean of last minus first signals (DM3) for live (top) and spoof (bottom)... 58

Figure 3-14 The ROC associated with Figure 3-13..............................................58

Figure 3-15 Mean of last minus first signals (DM3) for live (top) and cadaver (bottom).

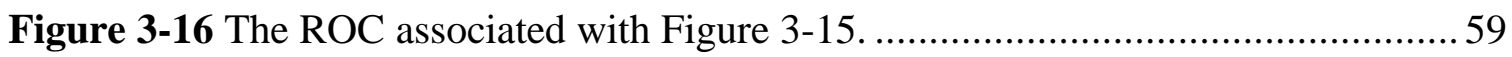


Figure 3-17 Percent change between last-first signal standard deviation (DM4) for live

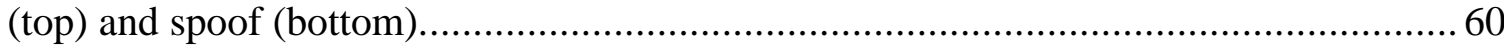

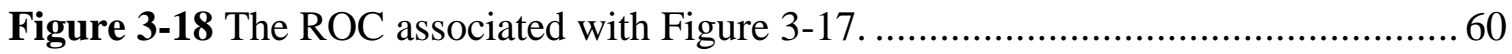

Figure 3-19 Percent change between last-first signal standard deviations (DM4) for live

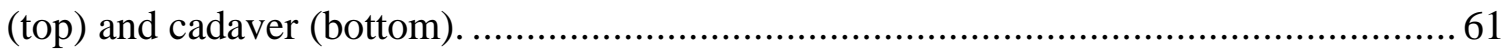

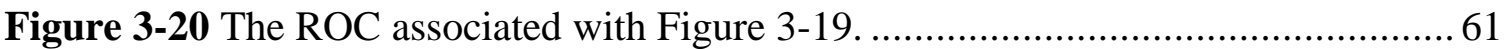

Figure 4-1 Layout of the back-propagation neural network. The inputs are the four dynamic and one static feature. Outputs (close to) +1 or -1 denote live or cadaver/spoof,

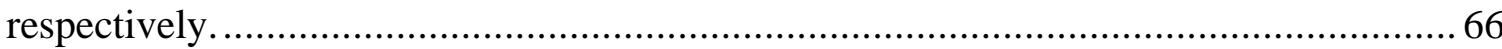

Figure 4-2 Plot of the sum-squared error during training. Error limit is set to 0.02. The number of epochs required for training was 9666 


\section{List of Tables}

Table 4-1: Classification of six live fingerprints of the test set, where a "1" indicates live and "0" cadaver/spoof. Last row shows polled results from all measures.

Table 4-2: Classification of six cadaver fingerprints of the test set, where a "1" indicates live and "0" cadaver/spoof. Last row shows polled results from all measures.

Table 4-3: Classification of six spoof fingerprints of the test set, where a "1" indicates

live and "0" cadaver/spoof. Last row shows polled results from all measures. 64

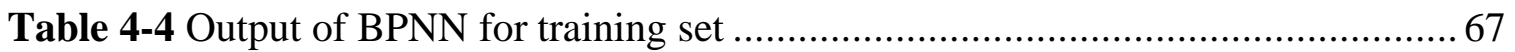

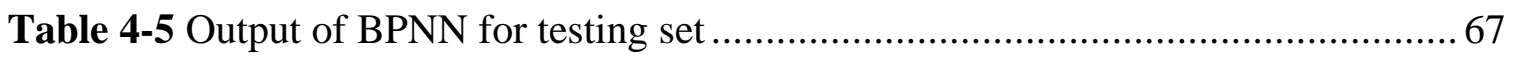



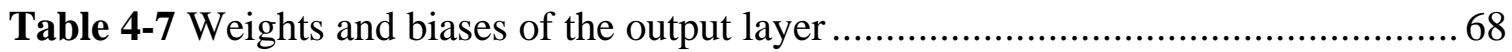


Chapter 1. Introduction and Background 


\section{1-1 Introduction}

Personal identification is a very important issue in today's complex, mobile and electronically networked societies. Identification can be in the form of either verification (checking a person against one enrollee) or recognition (finding out who a person is, by matching the acquired characteristics against a large database of enrollees). For proving one's identity, a unique characteristic should be offered. Typical automated methods require yielding an entity related to the person, like a key, card, a password (PIN), or a combination of those. The problem with these methods is that the authenticating entities can be stolen, forgotten or lost and cannot distinguish the authorized person from a felon possessing the authenticating entities. A solution to these problems is using physical characteristics of the person. Each person has set of unique physiological characteristics. An identifying biological measure is called a biometric. Among all biometrics, fingerprints are the oldest and most widely used [1].

\section{1-1-1 Project Statement}

Unfortunately, depending on the capturing technique, it is usually possible to fool automatic fingerprint identification systems by presenting a well-duplicated synthetic or dismembered finger. This project introduces a new method to determine the "liveness" or vitality of the finger presented in order to solve the spoof-attack problem. Perspiration of live fingers is detected as a sign of life, something that is absent in cadaver and spoof fingers. Using a sensor that is composed of an array of capacitors, this method identifies the vitality of a fingerprint by looking at a series of fingerprints captured during a 5second time frame and detecting a perspiration pattern over the human skin. Mapping two-dimensional images into one-dimensional signals, two ensembles of measures, namely static and dynamic measures, are extracted and used for classification. Static feature as well as temporal changes in dielectric mosaic structure of the skin is caused by 
perspiration in the fingerprint signals. Using these features, this algorithm quantifies the sweating pattern and makes a final decision about vitality of the fingerprint using a neural network trained by examples [2].

\section{1-2 Biometrics}

The method of identifying a person from his/her unique physiological/behavioral signature is called biometrics. Any biometric measure should have specific properties, including universality, uniqueness, and permanence. It also should be accepted by people and difficult to fool. Below is a list of the most commonly used biometrics [1]:

- Fingerprints

- Hand and finger geometry

- Hand vein patterns

- Ear geometry

- Face recognition

- Voice recognition

- Retinal scans

- Iris patterns

- Writing, typing and walking patterns

- DNA, odor

\section{1-3 Applications}

With increasingly cheap, fast, and widely available computing hardware and small, inexpensive sensors combined with new demanding applications like online 
transactions, attention is being focused on biometrics-based identification. Some examples of this fast growing field are:

- Controlling access to networks, including the Internet

- Computer logins

- Accessing confidential databases, such as medical records

- Tracking time and attendance

- Authorizing transactions, especially financial

- E-commerce and web banking

- Document encryption (using person's biometrics as key)

- Verifying identities at point of sale

- Using ATM and credit cards

- Controlling access to office buildings or homes

- Protecting personal property, like cars

- Preventing welfare and healthcare fraud

\section{1-4 Fingerprints and Related Fraud}

Among the introduced biometric measures, fingerprints are among the oldest and most widely used. Since 1960s, automated fingerprint identification systems (AFIS) have been widely deployed in law enforcement agencies [3]. Fingerprints are unique for each individual and each fingerprint is formed through embryonic development stages. Fingerprint captures are usually represented by the entire image, ridges, or features derived from the ridges called minutiae (consisting of ridge endings and bifurcations) [1].

During automatic verification, the claimant's fingerprint is compared against an enrollee fingerprint. Unfortunately, depending on capturing technique, it is usually possible to fool the device by presenting a well-duplicated synthetic finger or a cadaver finger in process of verification (see chapter 2). Some have suggested anti-spoofing 
measures based on physiologic features which may include measuring skin resistance, temperature, pulse-oximetry (blood oxygen measured by absorption of near infrared light and red light) electrocardiogram (electrical potential changes of cardiac activity versus time) and/or other physiological vitality indicators.

These measurements have the disadvantage that they are bulky and expensive. Furthermore, some of the features are easy to spoof. For example, the spoof finger can be coated with a material with similar electrical resistance as skin or it can easily be warmed to $37^{\circ} \mathrm{C}$ to fool the temperature sensor.

One expanded method of using physiologic features is described in US Patent 5,719,950: Biometric, Personal Authentication System. This design, patented on 2/17/1998, is a multi-modal biometric identification system with vitality tester [5]. The input to system comes from CCD camera (fingerprint scan), ECG electrodes (electrocardiogram of the claimant), LED and photo detector for pulse oximetry, and a temperature sensor. The system reads fingerprint for identification/verification (comparing to an enrollee) and uses skin temperature, pulse (both from ECG and optical readings, which should correlate), and oxygenation of blood for vitality measurement. If the fingerprint scan verifies the identity the claimant and if the second ensemble (vitality) readings fall into an acceptable range, then the claimant will be authenticated. In essence, the system is able to determine whether the live person is present while the fingerprint is being scanned. It also uses a PIN to narrow the search of the claimant characteristics against the enrollees' database. Automatic fingerprint identification system resistance to spoof fingers is performed by adding extra hardware which reads the vital signs from the claimant's hands.

A closely related project successfully carried out here at Biomedical Signal Analysis Lab (BIOSAL), WVU, uses pulse-oximetry in anti-spoofing hardware/software [4]. 
In this dissertation, we propose a new approach for spoof identification which is based on time-domain changes in a capacitively-captured image. The major advantage of our approach is that systems can become "spoof-proofed" by a simple software upgrade. 


\section{1-5 The Skin}

Skin is composed of three main layers: epidermis, dermis, and subcutaneous tissue. Epidermis houses sweat glands, hairs, sebaceous glands, and nails [6]. Each square inch of skin contains approximately 30 million cells, 100 fat glands, 600 sweat glands, and thousands of nerve endings, among other things [6].

Skin has many functions, including excretion of substances through sweat glands and absorption of lipid-soluble substances [7]. In addition, skin on the finger has a unique fingerprint pattern, which has been used as a method for identification. Skin's other functions include protection against toxins, sun, injuries as well as thermal regulation, to name a few [6].

\section{1-5-1 Epidermis}

The epidermis is usually thinner than other layers except in the palms and soles [7]. It is composed of the horny layer (dead epidermis) and the basal layer (living epidermis). The live cells produced in basal layer are constantly pushing the old cells up and away from sources of nourishment. Gradually, they loose their nuclei and turn into lifeless protein called keratin. The total cell life cycle takes 4 weeks [6]. 


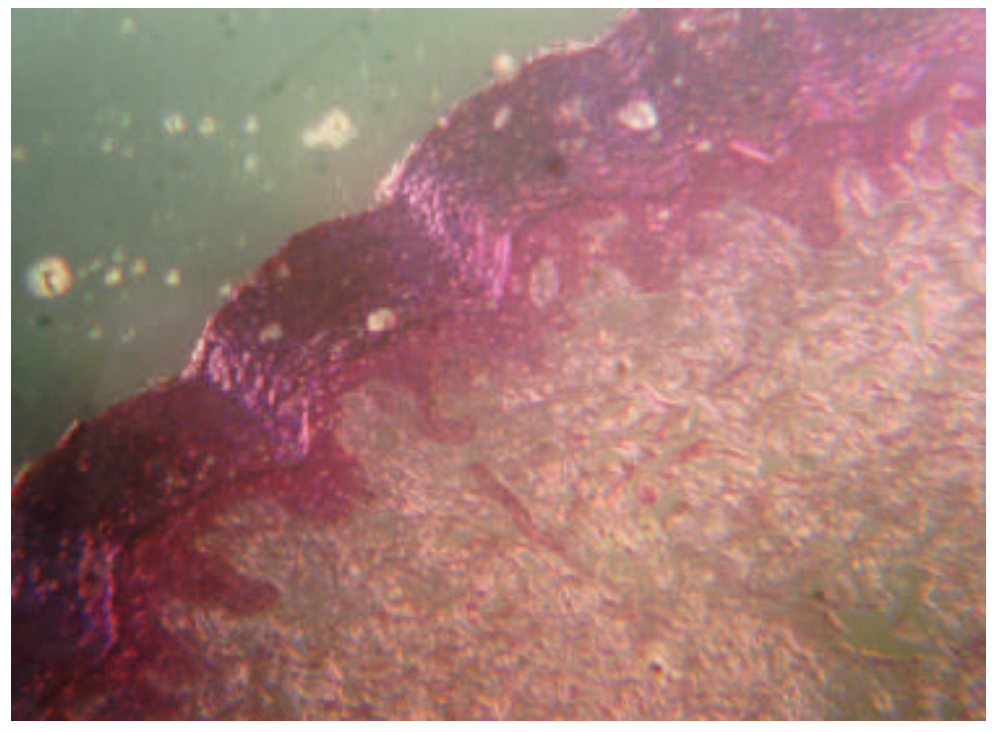

Figure 1-1 Fingertip skin histology using 80x magnification.



Figure 1-2 Schematic of general skin structure including various layers and estimated measurements. 
A histology from a cadaver finger at WVU and a schematic further illustrates the structure of the skin (Figures 1-1, 1-2). Important features include the ridge-to-ridge (which is similar to the pore-to-pore distance) and valley depth measures. For fingertips, the valleys are deeper and the ridge-to-ridge (and pore-to-pore) distance decreases towards the center.

\section{1-5-2 Dermis}

Dermis is composed of gel-like, elastic materials, water, and collagen [6]. In a cross-section, dermis includes finger-like connective tissue protrusions called "dermal papillae". In thick skin, like fingerprints, dermal ridges present the same unique pattern as epidermal ridges [7]. The dermis also contains sebaceous and sweat glands, tiny blood vessels and hair follicles. Sebaceous glands produce an oily substance called sebum. There are two types of sweat glands: apocrine and eccrine. The former produces body odor and the latter mainly functions as the body's temperature control system. Their highest concentration is in the palms, soles, fore head and underarms. Eccrine sweat glands especially respond to hot weather, physiological activity, emotional stress and taking spicy foods [6]. Perspiration is described in more detail in 1-6.

\section{1-5-3 Subcutaneous Tissue}

Subcutaneous tissue is another layer of conductive tissue below dermis that forms and retains fat as reserve fuel. It also produces vitamin D [6]. 


\section{1-5-4 Pores}

Pores are small openings of the sweat ducts in the skin surface. The sweat ducts originate from the subcutaneous layer and pass through dermis to the epidermis. Furthermore, extensive research shows that pore patterns are unique. Pores do not disappear, move, or spontaneously change over time [8].

Pore-to-pore distance is different from person to person, and varies depending on the location in the finger. However, on the average, our observations show that the poreto-pore distance is approximately $0.5 \mathrm{~mm}$. This agrees with Ashbaugh's model that considers frequency of 20.8 pores/cm on the ridge, or $0.48 \mathrm{~mm}$ distance between each pair of pores [8]. 


\section{1-6 Sweating}

Sweating is a main function of human skin that regulates human body temperature. One can define sweating as "active secretion of a watery fluid onto the body surface from either eccrine or apocrine sweat glands" [9]. Apocrine glands are of minor importance. This gland is responsible for body odor and is present at underarms, around nipples and navels and in genital area.

The main source of sweat is millions of eccrine glands that form a unique and advanced thermo-regulating system. The highest concentrations are found on palms, soles, forehead, and underarms [6]. The concentration ranges from $60 / \mathrm{cm}^{2}$ on thigh to $350 / \mathrm{cm}^{2}$ on forehead [9]. Sweating usually begins when the environment temperature increases approximately $1{ }^{\circ} \mathrm{C}$. Eccrine sweat gland is composed of a tubule coiled into a bolus 2-5 mm below skin surface in the sub dermal tissue.

\section{1-6-1 Perspiration Control}

Integrated control of perspiration is done by the hypothalamus. Using the sympathetic system, spinal chord segments T2-T4 stimulate sweat glands on head and neck, T2-T8 upper limbs, T6-T10 trunk, and T11-L2 lower extremities. Central control is affected by inputs from skin and core temperature. Sweating is also controlled by local skin temperature. Maximal rate of sweating is also a function of sex, age, conditioning, season, and diet. It is also observed that isolated sweat glands continue to secrete when the temperature goes above $43{ }^{\circ} \mathrm{C}[9]$. 


\section{1-6-2 Sweating Disorders}

There are several disorders associated with sweating, namely:

1- Hyperhidrosis: This term is applied to excess sweating conditions. It can be local or systemic. The localized hyperhidrosis can be caused by emotions (especially in axillary, palmar, and plantar regions). The hyperhidrosis of the palm maybe very severe. Another cause for local hyperhidrosis can be "gustatory", like when a person consumes hot and spicy foods. There is also a systemic hyperhidrosis which happens all over the body. It is caused by disturbance in autonomic nervous system, disorders in thermo-regulation, or hypersensitivity to stimulation.

2- The opposite situation is "Anhydrosis" which is lack of sweating, even when exposed to stimulants like heat or chemical agonists. It is rare and can be caused by various diseases or damages. It can be segmental, localized, or systemic [9].

\section{1-6-3 Composition of Sweat}

Eccrine glands produce majority of the sweat. Sweat has mainly non-organic and small organic components, both of which vary greatly with sweating rate. Most of sweat electrolyte concentrations are less than that of extra cellular fluids. The major solute, sodium chloride, is usually in range of 15-50 mM. Other existing solutes in sweat include: $\mathrm{HCO}_{3}$, urea, potassium and hydrogen (normally higher in sweat compared to extra-cellular fluid), very little Magnesium and $\mathrm{PO}_{4}$, glucose, lactate, amino acids, and proteins and enzymes. The normal $\mathrm{pH}$ of sweat is less than 5. In some sweating disorders, concentration of solutes, especially sodium chloride, may differ (usually becomes higher) [9]. 


\section{1-7 Electrical Model of Skin}

The most important characteristics of skin are its impedance and capacitance. Skin has a complicated structure and has a complex response to electrical signals. This response is highly dependent on the original condition of skin, external stimulants, and the intensity of the applied electrical signal. Under small electric fields, the skin's voltage-current characteristic is (quasi) linear and symmetric $[10,11,12]$. However, for increases in the amplitude of the electrical stimulus (either voltage or current), the characteristics become nonlinear and asymmetric, pain starts and the skin breaks down with a sudden decrease in impedance [11]. Studies also show the frequency dependence of skin, in which it acts like a low pass filter for frequencies up to $1 \mathrm{kHz}$ [11].

Our experiments on live and dead fingers show a difference in the frequency response, especially in the dynamism of the response (Appendix B). It is also important to note that skin electrical characteristics are time dependent (i.e. during an experiment) [12].

Considering the skin as a porous membrane, the lipid non-conductive parts can be modeled as a capacitor and the conductive sweat ducts as parallel resistor-capacitors (Figure 1-3) [13, 10].
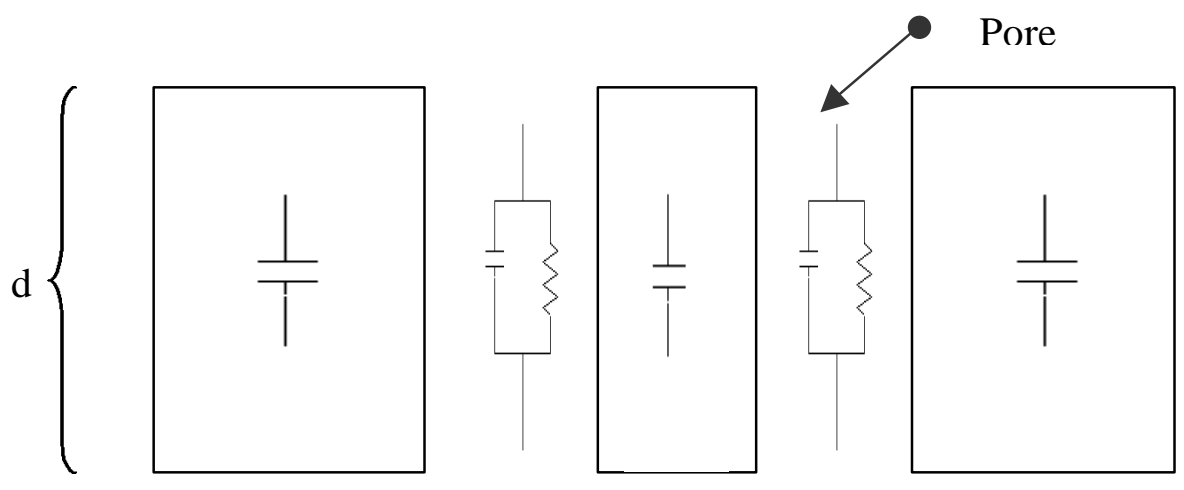

Figure 1-3 Cross section of (the outer layer of) skin as a parallel combination of resistors and capacitors 
The following paragraphs derive capacitance of a specific area of skin, say A. This is a simplified model and only takes into account the outer layer of skin. Let $A_{p}$ be the total area of pores filled with sweat, $A_{s}$ the total area of lipid (solid skin), $d$ the thickness of the layer, and $\varepsilon_{0}, \varepsilon_{\mathrm{w}}$, and $\varepsilon_{\mathrm{s}}$ the permittivity of free space, dielectric constant for sweat, and dielectric constant for lipid, respectively. Then, the capacitance of the pores is given by:

$$
C_{p}=\frac{A_{p} \varepsilon_{w} \varepsilon_{0}}{d}
$$

The capacitance of the skin itself, without the contribution of the pores, is:

$$
C_{s}=\frac{A_{s} \varepsilon_{s} \varepsilon_{0}}{d}
$$

Since $\mathrm{A}=\mathrm{A}_{\mathrm{p}}+\mathrm{A}_{\mathrm{s}}$, a porosity factor $\mathrm{p}$ and can be written as:

$$
A_{p}=p A
$$

$$
A_{p}=(1-p) A
$$

Therefore, the total capacitance is calculated as:

$$
\begin{gathered}
C=C_{p}+C_{s}=\frac{p A \varepsilon_{w} \varepsilon_{0}+(1-p) A \varepsilon_{s} \varepsilon_{0}}{d} \\
C=\frac{\varepsilon_{0} A}{d\left[\varepsilon_{w} p+\varepsilon_{s}(1-p)\right]}
\end{gathered}
$$

It is interesting to note that one can calculate the porosity of the membrane by arranging the above formula [13]: 


$$
p=\frac{\frac{d C}{\varepsilon_{0} A}-\varepsilon_{s}}{\varepsilon_{w}-\varepsilon_{s}}
$$

The (relative) dielectric constant of water (main ingredient of sweat) is $\sim 80$, and for lipids $\sim 2-3$. For the lipid-corneocyte matrix of stratum corneum (outer-most layer of skin), an intermediate value of 15-30 is reasonable for hydrated lipid bilayers [10]. However, since there are large differences between the dielectric constants of sweat, moist skin, and dry skin, one should expect large differences in the measured capacitance across skin when comparing perspiring pores and drier regions.

Literature on skin conductance shows a direct link between sweat ducts, sweating, and electrical conductance. The sweat ducts are the main current paths for current and ionic flow, giving the skin (epidermis) a mosaic electrical structure [11, 12]. Experiments with microelectrodes confirm that skin conductance peaks at the pores. In addition, the ratio of $\mathrm{Z}_{\max } / \mathrm{Z}_{\min }$ (non-pore/pore area) decreases by slight changes in temperature while the average impedance drops. This change is considered a direct consequence of an increase in sweat glands activity [11].

These results correspond with our findings in the capacitance domain. The hypothesis of this document is that the fingertip skin, in conjunction to the fine capacitive grid of a fingerprint scanner, has a larger capacitance for those cells above the moister sweat ducts given the much higher dielectric constant of sweat relative to drier skin. As the skin continues to perspire and the sweat diffuses towards the drier regions between the pores, the ratio of $\mathrm{C}_{\max } / \mathrm{C}_{\min }$ will decrease. This hypothesis is further described in Chapter 2. 


\section{1-8 Fingerprint Scanner}

Fingerprint scanners use different mechanisms for capturing the fingerprint, each having its own advantages and disadvantages. Most of the existing scanners use either ultrasonic imaging (high quality images but expensive and bulky), pressure sensor array (small integrated sensor but low resolution and expensive), optical imaging (most widely used, but requires light source, prism, lens, imager, and is expensive and generally bulky), or capacitive proximity sensor arrays [14]. The last category, though relatively new and still facing problems, seems to be a promising technology.

Capacitance sensors are composed of a 2-D array of capacitors, using standard CMOS processing (Figure 1-4) [15]. These sensors are exposed to direct fingertip contact. A thin but very tough and resistant dielectric (passivation) layer separates the touching surface from the integrated circuit. This layer plays a very important role since it must be resistant to skin oils, moisture and chemicals that can migrate to the silicon (including sodium ion and chlorine/chloride ions that can corrode metals) as well as surface scratches and physical wear and tear, and electrostatic discharge. It also should be thin enough and have high dielectric constant so the capacitance between sensor and finger, $\mathrm{C}_{\mathrm{f}}$, will dominate the stray capacitance between sensor and substrate (and/or adjacent cells), $\mathrm{C}_{\mathrm{s}}$. The sensitivity can also be increased with enlarging the sensor plates and decreasing the parasitic capacitance by altering the thickness and dielectric constant of lower dielectrics as well as other established capacitive shielding techniques (Figure 14) $[14,15]$. 


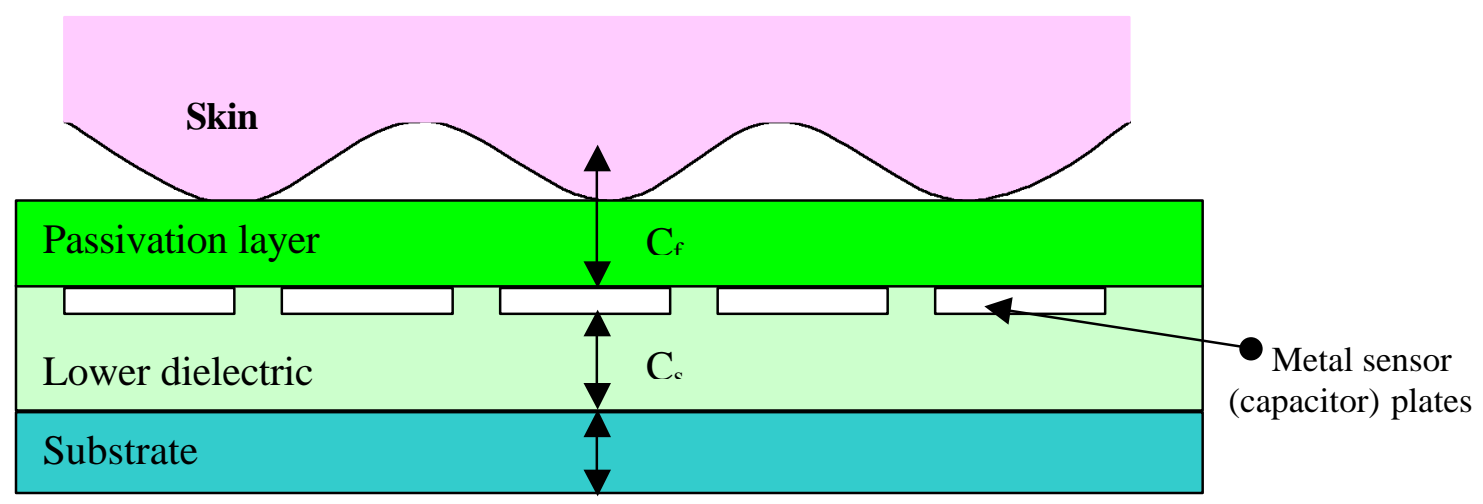

Figure 1-4 Typical capacitive sensor architecture

Therefore, device sensitivity is proportional to $\mathrm{C}_{\mathrm{f}} / \mathrm{C}_{\mathrm{s}}$. The finger acts as the upper grounded capacitor plate [14, 15], where it is considered as an equipotential surface [15]. Another mode of operation can be summarized as follows. The electric flux that originates from a sensor's metal plate loops back onto the device surface and terminates into an adjacent ground grid surrounding each cell. If a ridge from the skin interrupts the flux, it affects the deposited charge and hence the fringe capacitance. In each case, the sensing range is short and capacitance decreases sharply as the distance from the surface of the device to the finger increases [15]. In essence, these devices are most sensitive to the parts of the fingertip which touch the sensor, i.e. the ridges. Each sensor's measured capacitance is translated into a grayscale level in the corresponding bitmap image of the captured fingerprint through a special circuitry The role of dielectric constants is an important aspect of capacitive sensing and is the underlying principle for our vitality detection algorithm. If the skin in contact with the sensor is moist, then, because of very high dielectric constant of sweat, the underlying sensor will yield a much higher capacitance, resulting in a darker (saturated) spot on the captured image. The basic calculations of capacitance for a typical sensor cell for Veridicom FPS 100 are presented below. 


\section{1-8-1 Modeling of the Veridicom FPS 100 Sensor Cell}

This research uses the Veridicom (Santa Clara, CA) FPS 100 capacitive fingerprint. One can model each sensor plate as being coated with two layers of dielectric, the passivation layer and the layer adjacent to it, whether it be air, skin (moist or dry) or a thin film of sweat. The capacitance of each cell has a $1 / d$ behavior. The characteristic length of sensitivity is of the order of the 'ground grid to cell' gap and 'sensor layer to substrate' spacing. Therefore, only the effect of a very thin layer adjacent to the passivation layer is taken into account

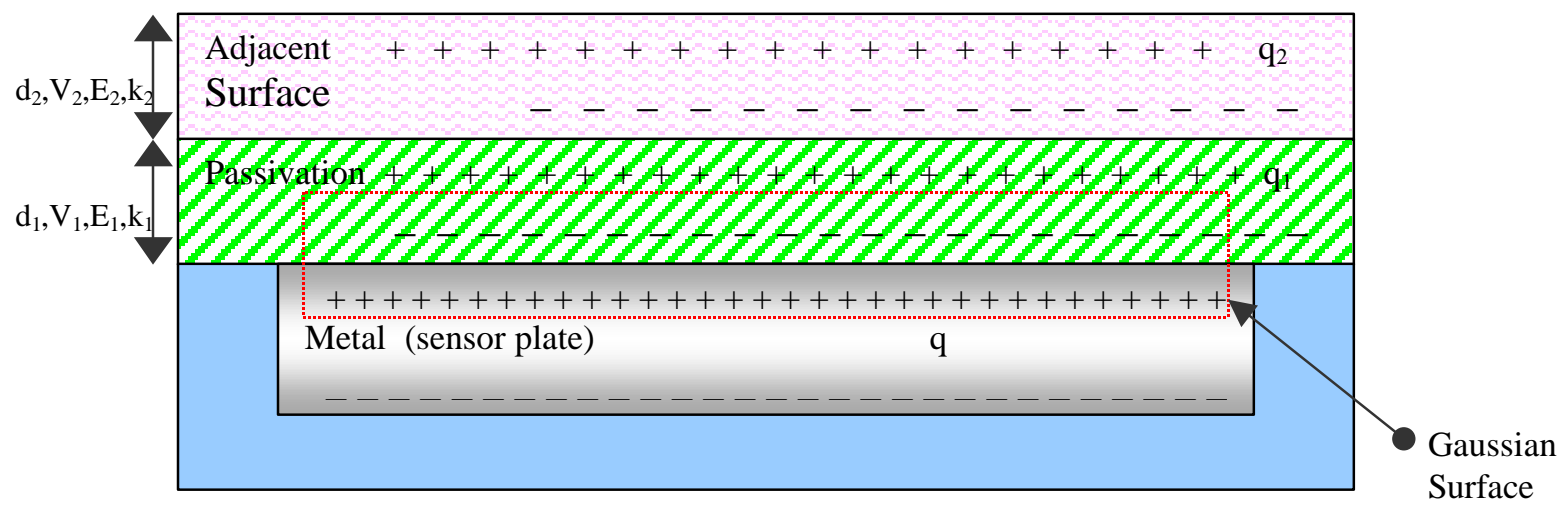

Figure 1-4 Schematic used in calculating capacitance detected by the sensor.

Considering Gauss' law:

$$
\oint_{A} \varepsilon_{0} \vec{E} \bullet d \vec{A}=q
$$

Where $\varepsilon_{0}$ is the permeability of free space, $\mathbf{E}$ the electric field vector, $\mathrm{d} \mathbf{A}$ the Gaussian surface normal differential vector, and $\mathrm{q}$ is the net charge inside the surface. Using the depicted Gaussian surface for the first layer of dielectric (passivation), $\mathrm{E}_{1}$, the electric field inside the passivation layer, can be written as: 


$$
\begin{gathered}
\varepsilon_{0} E_{l} A=q-q_{l} \\
E_{l}=\frac{q-q_{l}}{\varepsilon_{0} A}
\end{gathered}
$$

where $\mathrm{q}$ and $\mathrm{q}_{1}$ are the charges included in the Gaussian surface with the base area of $\mathrm{A}$. Introducing the dielectric constant concept the above can be written as:

$$
E=\frac{q}{\varepsilon_{0} k_{l} A}
$$

since

$$
V_{l}=E_{1} d=\frac{q d_{1}}{\varepsilon_{0} k_{l} A}
$$

where $d_{1}$ is passivation layer thickness. The Gaussian surface can be further expanded into the second dielectric layer (adjacent skin). With similar calculations:

$$
V_{1}+V_{2}=\frac{q d_{1}}{\varepsilon_{0} k_{1} A}+\frac{q d_{2}}{\varepsilon_{0} k_{2} A}
$$

where $\mathrm{d}_{2}$ is adjacent surface (outer layer of skin) thickness. The resulting capacitance is defined as:

$$
q=C\left(V_{1}+V_{2}\right)
$$

and results in: 


$$
C=\left(\frac{d_{1}}{\varepsilon_{0} k_{1} A}+\frac{d_{2}}{\varepsilon_{0} k_{2} A}\right)^{-1}
$$

or

$$
C=\frac{\varepsilon_{0} A k_{1} k_{2}}{k_{1} d_{2}+k_{2} d_{1}}
$$

For the Veridicom FPS 100 fingerprint scanner, $\mathrm{d}_{1}$ equals to 5000A (0.5 micron) and $\mathrm{k}_{1}$ is greater than 7 [14]. Considering a sensed depth of $\mathrm{d}_{2}$ equal to 2 microns for the adjacent skin [15], the multiplying factor $k_{1} k_{2} /\left(k_{1} d_{2}+k_{2} d_{1}\right)$ for water (sweat), normal skin, dry skin, and air, using their nominal dielectric constants (see 1-7) is $10.4,6.2,1.2$, and 0.5 , respectively. One can see that the lower $\mathrm{d} / \mathrm{k}$, the higher the capacitance. Therefore, the capacitance for saturated skin (sweating pore) is nearly 10 times larger than that of dry skin. For further details about basic capacitance calculations, please see [16], [17]. 


\section{1-8-2 Sensor Cell Circuit}

For each individual sensor plate, the fingerprint scanner, Veridicom FPS 100, has the circuit shown in Figure 1-5. It consists of a circuit to charge/discharge each sensor (capacitor) plate and a subsequent sample and hold circuit. The measured capacitances are then read back into the host computer through a series of row-column sweeps.

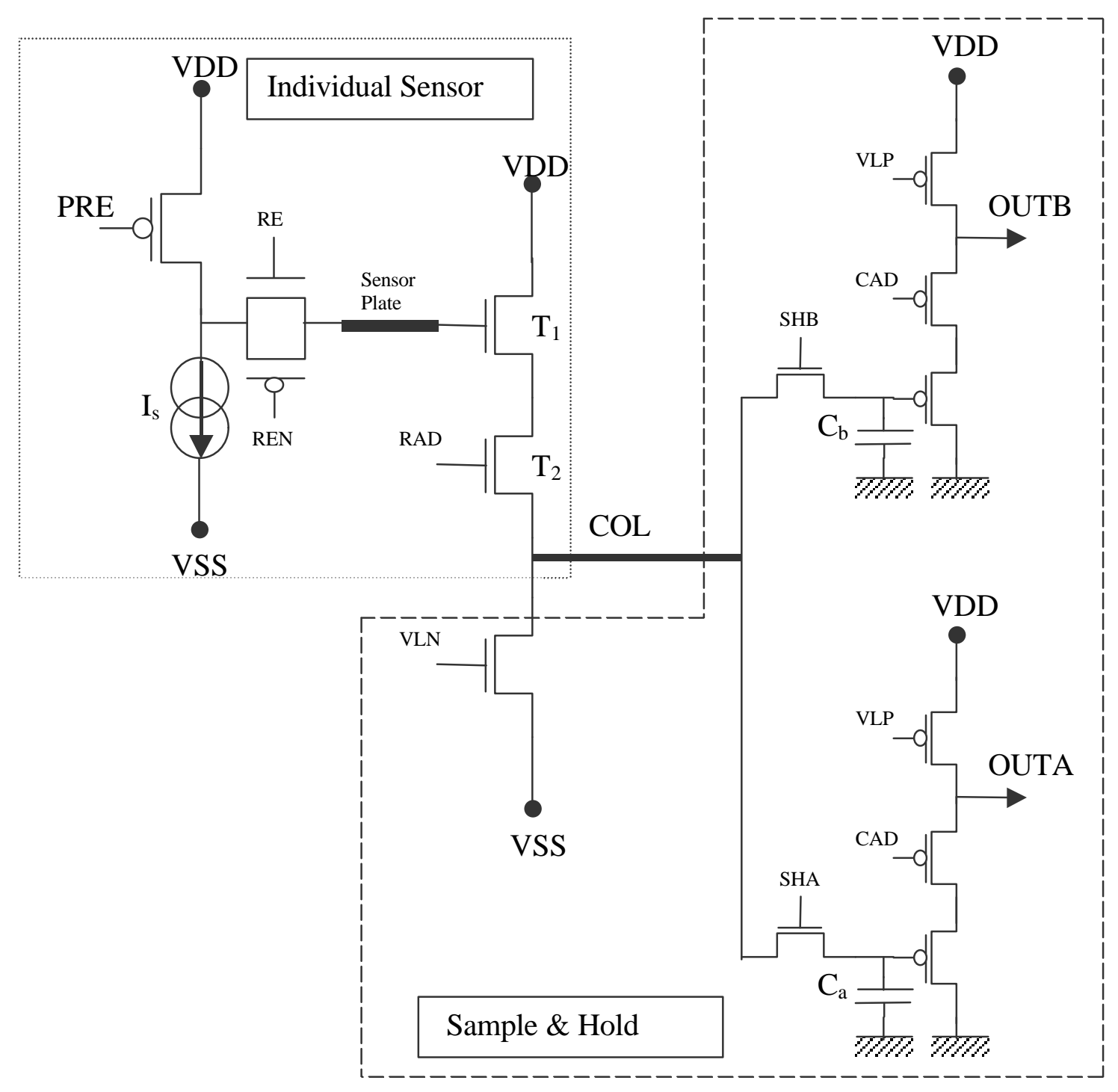

Figure 1-5 Veridicom FPS 100 sensor cell with sample and hold circuit [14]. 
The voltage of the sensor plate is proportional to the deposited charge due to the capacitance generated the adjacent finger. This voltage is measured and samples by the circuit.

The following describes the function of the circuit depicted in Figure 1-5.

In each cycle, RE and RAD select the addressed cell. PRE pre-charges the sensor plate by VDD. $T_{1}$ buffers the resulted voltage through the very high input resistance of its gate and as a source follower. This voltage is gated to $\mathrm{C}_{\mathrm{a}}$ by SHA pulse. After releasing PRE, the current source $I_{S}$ drains the deposited charge during a fixed period of time. Then, the new plate voltage is gated to $\mathrm{C}_{\mathrm{b}}$ by SHB pulse. Refer to Figure 1-6 for more details about the timing of cell read cycle.

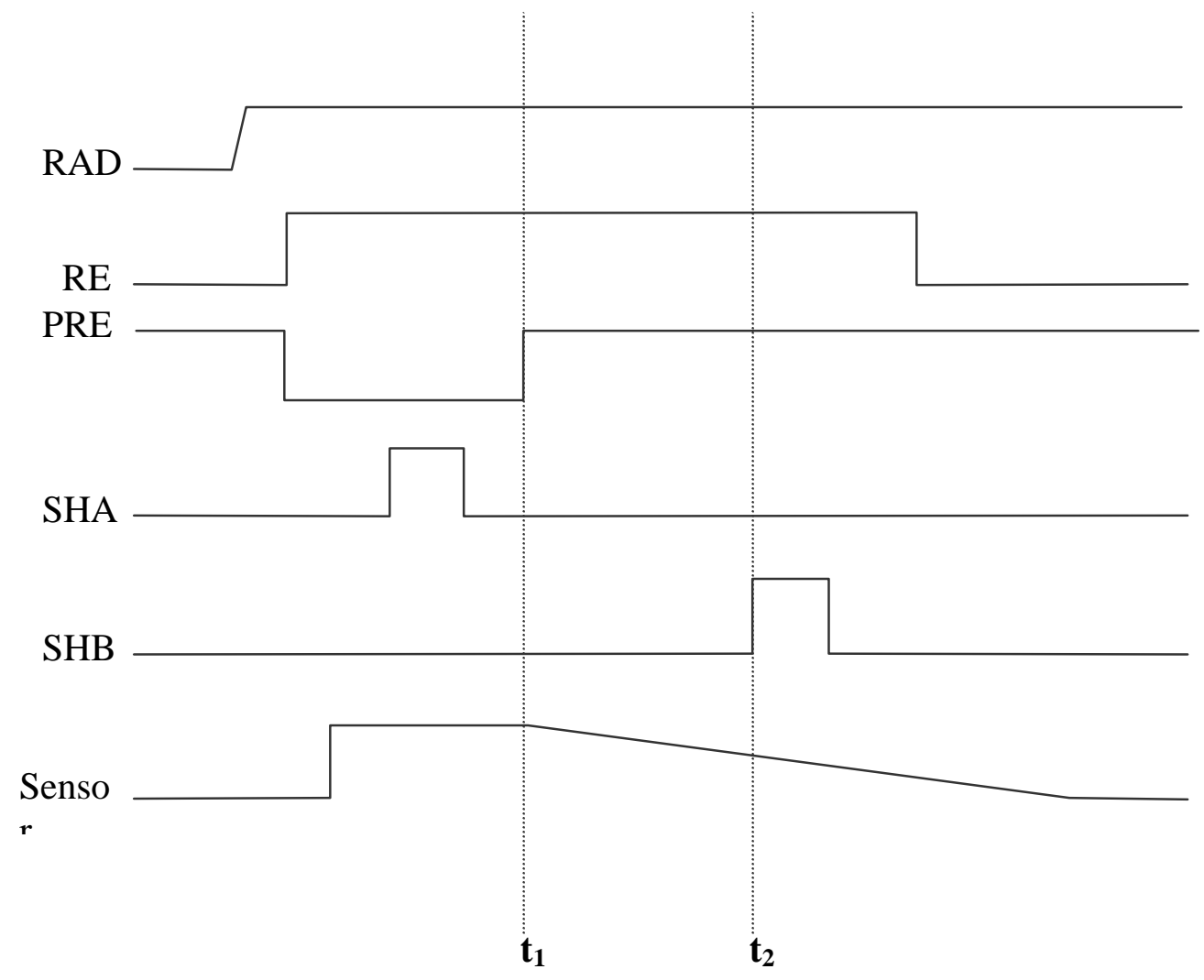

Figure 1-6 Sensor read cycle timing [14].

A subsequent circuit subtracts $\mathrm{V}_{\mathrm{b}}$ from $\mathrm{V}_{\mathrm{a}}$. By doing so, the effects of the variations on threshold voltages of $t_{1}$ and $t_{2}$ are removed and the net output will be 
proportional to the sensed capacitance [14]. This effect can be depicted as below. If we consider the actual expected voltages for beginning and end of sample and hold period to be $V_{1}$ and $V_{2}$ according to the following relationship, then:

$$
\begin{aligned}
& V_{a}=V_{1}+V_{\text {Noise }} \\
& V_{b}=V_{2}+V_{\text {Noise }}
\end{aligned}
$$

Moreover, for actual stored charge, we can write:

$$
\begin{gathered}
q_{1}=C V_{1} \\
q_{2}=C V_{2} \\
q_{2}-q_{1}=C\left(V_{2}-V_{1}\right) \\
q_{2}-q_{1}=C\left[\left(V_{2}+V_{\text {Noise }}\right)-\left(V_{1}+V_{\text {Noise }}\right)\right] \\
q_{2}-q_{1}=C\left(V_{b}-V_{a}\right)
\end{gathered}
$$

since

$$
q_{1}-q_{2}=I_{s}\left(t_{2}-t_{1}\right)
$$

then

$$
C\left(V_{a}-V_{b}\right)=I_{s}\left(t_{2}-t_{l}\right)
$$

so

$$
C=\frac{I_{s}\left(t_{2}-t_{1}\right)}{V_{a}-V_{b}}
$$

or 


$$
C=\frac{\text { const }}{V_{a}-V_{b}}
$$

VLN and VLP are constant and can be controlled through software. These voltages change the general brightness and contrast of the captured fingerprint. More detailed information about the sensor can be found in Appendix A. 


\section{1-9 Artificial Neural Networks}

Explanations of brain's mechanism and thinking process have been around since the time of Plato and Aristotle. However, analytical neural models did not appear until recently. One of the first of such models was offered by McCulloch and Pitts in 1943. Other theories were followed by Farley and Clark, Rosenblatt, Widrow and Hoff, and others [18].

Biological neural systems are the central control of animal's behavior, namely sensory and motor functions, internal processing, and thinking. Artificial Neural Networks, or neural nets, try to mimic these functions. They are analog systems of massively parallel, interconnected, simple, nonlinear processing elements. One of the major advantages of neural nets over digital computers is the ease of taking into account high-order statistical relationships of stochastic data. "Training" of such systems utilizes the principle of reward and punishment by back-propagating the needed information for altering structures of interconnections and strength or weights of these interconnections. Neural nets are considered a significant breakthrough in artificial intelligence [18].

A simple neuron, as depicted in Figure 1-7, sums the weighted inputs plus a bias and passes the result through a nonlinearity (usually sigmoid, but sometimes linear or a hard limiter). 


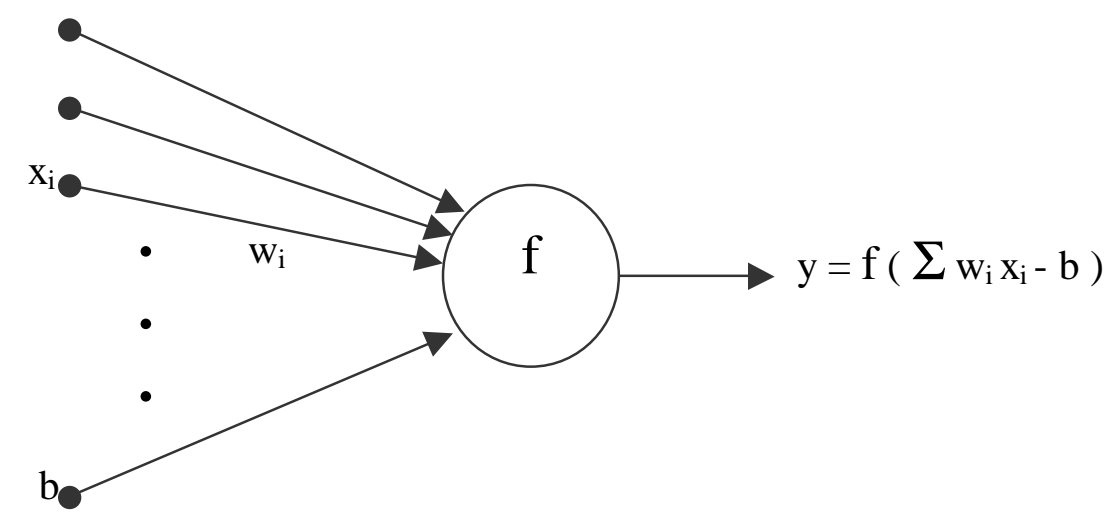

Figure 1-7 A simple neuron, consisting of weighted inputs $\mathrm{w}_{\mathrm{i}} \mathrm{x}_{\mathrm{i}}$, a bias $\mathrm{b}$, and nonlinear function $\mathbf{f}$.

Neural net classifiers are non-parametric and make weaker assumption for shapes of distributions compared to their statistical counterparts. Therefore, they may be more robust when the distributions are caused by nonlinear and strongly non-Gaussian processes [19].

In this project, a back propagation neural net (three layer perceptron), with sigmoid nonlinearity, was used as classifier (Figure 3-1). These networks can approximate any function with a finite number of discontinuities [20]. To be more specific, Kolmogorov proved in his theorem that "any continuous function of $\mathrm{N}$ variables can be computed using only linear summations and nonlinear, but continuously increasing, functions of only one variable". Therefore, a three-layer perceptron with sigmoid nonlinearity can compute the desired continuous classifying function. This also can be viewed as the ability to form arbitrary decision regions. Note that this theorem only proves the "existence" of the ideal answer and efficient training rules are topic of current research [19]. After proper training by pairs of input-targets, this type of network yields very good performance in terms of generalizing the learned rule to inputs that it has never been exposed to before [20]. 
The training algorithm is a very important aspect of the neural network. The standard algorithm for back-propagation network is gradient descent. Unfortunately, this algorithm, though the best available, does not necessarily minimize the error function globally giving an optimal answer and often leads the network to a local minimum. Therefore, one has to provide the algorithm with random initial weights, biases, and the desired precision as an exit condition, among other things. Usually, the lower the precision, the better the generalization.

Here is a summary of the basic back-propagation gradient descent algorithm. This is an iterative gradient computing method that tries to minimize the mean square error between actual output of the multi-layer feed-forward perceptron and the desired output through partial differentiation, since differentiable nonlinearities (sigmoid logistic) are used.

\section{Basic Steps:}

1. Initialize weights and biases with random values.

2. Present the network with desired input-outputs. For classifiers, usually bipolar targets values (say +1 and -1 ) are used.

3. Calculate the related output.

4. Use the following recursive algorithm, working from output back to the first layer. The weights are adjusted according to:

$$
w_{i j}(t+1)=w_{i j}(t)+\eta \delta_{j} x_{I}^{\prime}
$$

where $w_{i j}(t)$ is the weight from neuron $i$ to the input of the neuron $j$ at time $t$, $\mathrm{X}_{\mathrm{i}}^{\prime}$ is the output of neuron $\mathrm{i}$ (or an input, for the first layer) and $\eta$ is the gain. $\delta_{\mathrm{j}}$ is the error for neuron $\mathrm{j}$. For output layer:

$$
\delta_{j}=y_{j}\left(1-y_{j}\right)\left(d_{j}-y_{j}\right)
$$


where $y_{j}$ is the actual output (Figure 1-7) and $d_{j}$ is the desired output. For hidden layer:

$$
\delta_{\mathrm{j}}=\mathrm{x}_{\mathrm{j}}\left(1-\mathrm{x}_{\mathrm{j}}\right) \underset{\mathrm{k}}{\sum} \delta_{\mathrm{j}} \mathrm{w}_{\mathrm{jk}}
$$

Biases are adjusted in the same way (one can think of a bias as another input for each neuron with constant value of 1). It is possible to speed-up convergence by adding a momentum term $\alpha$, which is a positive number less than 1, as follows:

$$
w_{i j}(t+1)=w_{i j}(t)+\eta \delta_{j} x_{i}^{\prime}+\alpha\left[w_{i j}(t)-w_{i j}(t-1)\right]
$$

5. Go to step 2 and repeat this loop until the desired precision (or a maximum number of iterations) has been reached.

This algorithm has been found to do very well in most cases. Usually, the error for input vectors not contained in training set is slightly higher [19]. 
Chapter 2. Methods and Materials 
The goal of this research is to develop a non-invasive method that would differentiate between live fingerprints and cadaver/spoof fingerprints using the Veridicom FPS100 capacitive solid state scanner. An ideal system would utilize measures that would not require any additional hardware or major system reconfigurations.

\section{2-1 Methodology}

Initial work focused on potential measurements of physiologic differences. This portion of the work included experimental measurement of the frequency response of dead and live fingers [Appendix B], and study of the effects of changing device parameters, including discharge current and discharge time. The major difference seen between scans was dependent on the moisture content of the scanned material. Further analysis showed that the higher the dielectric constant of the material, the darker the captured image.

The next stage of our work focused on the moisture content of the scanned object. An important contributor to the moisture on the surface of finger is natural perspiration, which begins from the openings of sweat ducts to skin, or pores, and diffuses over the skin. This phenomenon was visually observed both by the scanner and by studying live fingers under an optical microscope. Due to obvious visual differences in the temporal scans of living and cadaver fingers, much of the research focused on developing image processing algorithms to quantify the physiologic process of sweating. The devised method identifies the vitality of a fingerprint by processing two fingerprints captured during a five-second time frame.

The routine first goes through an image processing stage, including noise reduction and contour extraction. Then, the captures are transformed into signals, where 
the two-dimensional image is mapped into one-dimensional signal. The temporal change of sweating pattern of the skin over the sensor demonstrates itself in these signals. Two types of features are extracted: (1) those detectable within the same signal, or static, and (2) those observed in temporal transition from one signal to the next (from the same finger), or dynamic.

A set of eighteen live, eighteen cadaver, and eighteen spoof finger scans were utilized for developing the algorithm. The methods are described in more detail in the next sections.

\section{2-1-1 Hardware}

The Veridicom (Santa Clara, CA) FPS100 was used as fingerprint capturing device. It was connected via USB port to a HP Vectra running a 233 Pentium (for cadaver finger captures). The same scanner was used in conjunction with a Dell OptiPlex Gxi running a 233 Pentium for live and spoof fingerprint captures.

\section{2-1-2 Software}

Software provided with the fingerprint scanner was used, including dgrabusb.exe, which captures a tiff image from the scanner connected to the USB port with specified discharge time and discharge current, and cfltr.exe which extracts a binary image from the original grayscale scan. Matlab5 was used for all processing and computation.

\section{2-1-3 Training and Test Set}

The training and test set includes 18 set of fingerprint images from individuals (live), 18 from cadavers, and 18 from spoofs. The live sets are from eighteen different individuals mainly in the age group of 20-29. The eighteen spoof sets were developed 
from play dough using multiple casts. Approval to perform data collection for live and spoof fingerprints was obtained from the Institutional Review Board (IRB protocol HS \# 14517). Cadaver work was approved by IRB protocol HS \# 14239 and was performed at WVU Musculoskeletal Research Center. The cadaver sets include fingerprints from two freshly harvested unfrozen fingers, and the rest are captured from different fingers from frozen cadavers for total of four individuals.

\section{2-1-4 Spoof Development}

Many materials yielded precise replicas of the fingerprint. However, since they were hydrophobic, they did not image on the sensor unless immersed in water. Furthermore, because the water settles in valleys, generally the spoof finger gave a negative scan. Materials tested include: light bodied Permalastic (polysulfide, type 3), Extrude XP (polyvinylsiloxane, type 0), light bodied polysiloxane type 3, latex, and a range of ordinary (toy) materials including Silly Putty®, Gak's Alive ${ }^{\mathrm{TM}}$, Icky Poo®, and Play-Doh®. Surprisingly enough, the best results in term of sensor image quality were obtained from play dough spoofs using rubber-based casts, due to the fact that the material is water-based. The best results obtained from high precision materials are presented here and are compared with the play dough spoof (Figure 2-1). The first two were soaked in water for two weeks.
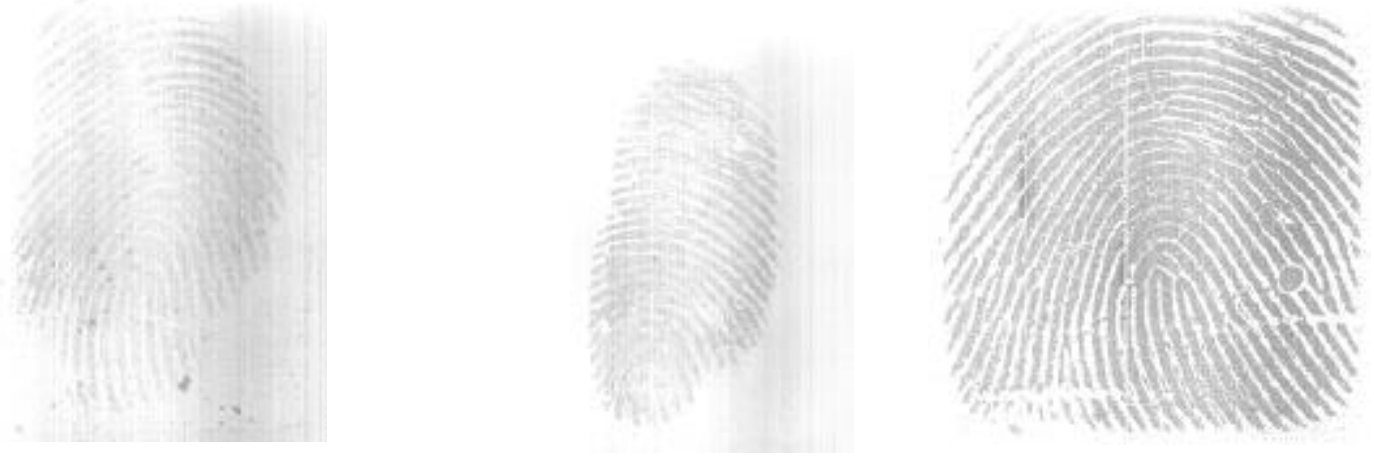

Figure 2-1 Soaked rubber based spoof (left and center), and play dough spoof (right) 
In addition, the play dough spoofs were tested using the new sensor and Virtual Console v 2.76 software (Veridicom, Santa Clara, CA). The play dough replicas of the real, enrolled fingers were accepted as true match even with the security level set to maximum. 


\section{2-2 Description of Physiologic Phenomenon:}

As mentioned in the introduction, this algorithm is based on the physiologic process of the perspiration arising in the pores, diffusing across the ridges. (Figures 2-2 and 2-3)

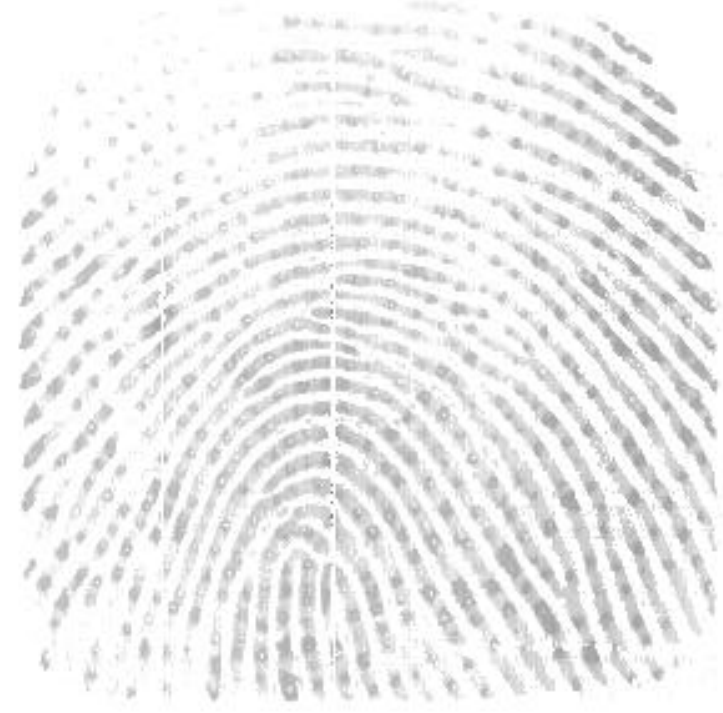

Figure 2-2 Live fingerprint @ $\mathrm{t}=0$

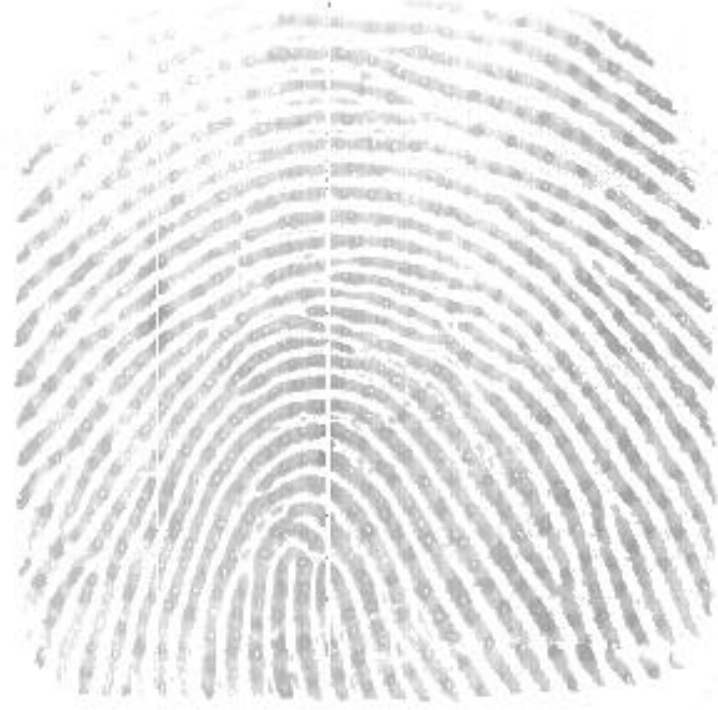

Figure 2-3 Live fingerprint @ $\mathrm{t}=5$

Inspection of the live fingers under optical microscope shows that perspiration from the pores takes place quickly. One can see two important features in the sweat formation (from the sequence of scanned fingerprints):

First, perspiration starts from the pores, either completely covering them or leaving the pore as a white (dry) dot in center of the sweating source. Typically the first scan will look "patchy" due to this process and has formed the basis of our static approach for classification. 
Second, the sweat diffuses along the ridges in time, making the semi-dry regions between the pores moister or darker in the image. Unless the skin is extremely dry, the pore region remains saturated while the moisture (sweat) spreads towards drier parts. This event formed the basis of our dynamic approach. Figure 2-4 is nine complete scans over five seconds (from top left to bottom right).
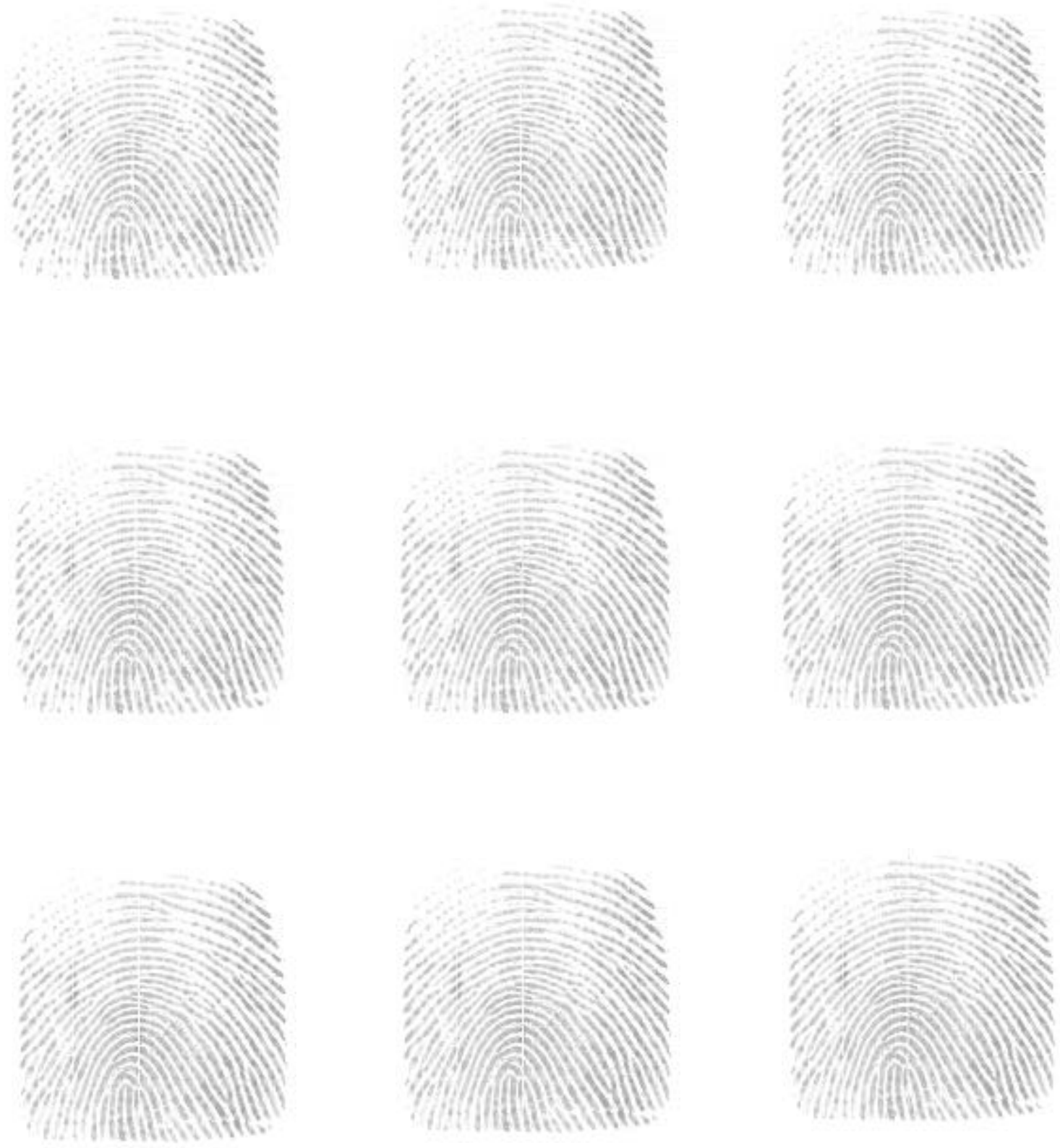

Figure 2-4 Nine scans (from left to right and top to bottom) collected over 5 seconds. 
The perspiration process does not occur in cadaver or spoof fingers. Figures 2-5 and 2-6 are first and last scans from cadaver and spoof fingers for comparison.
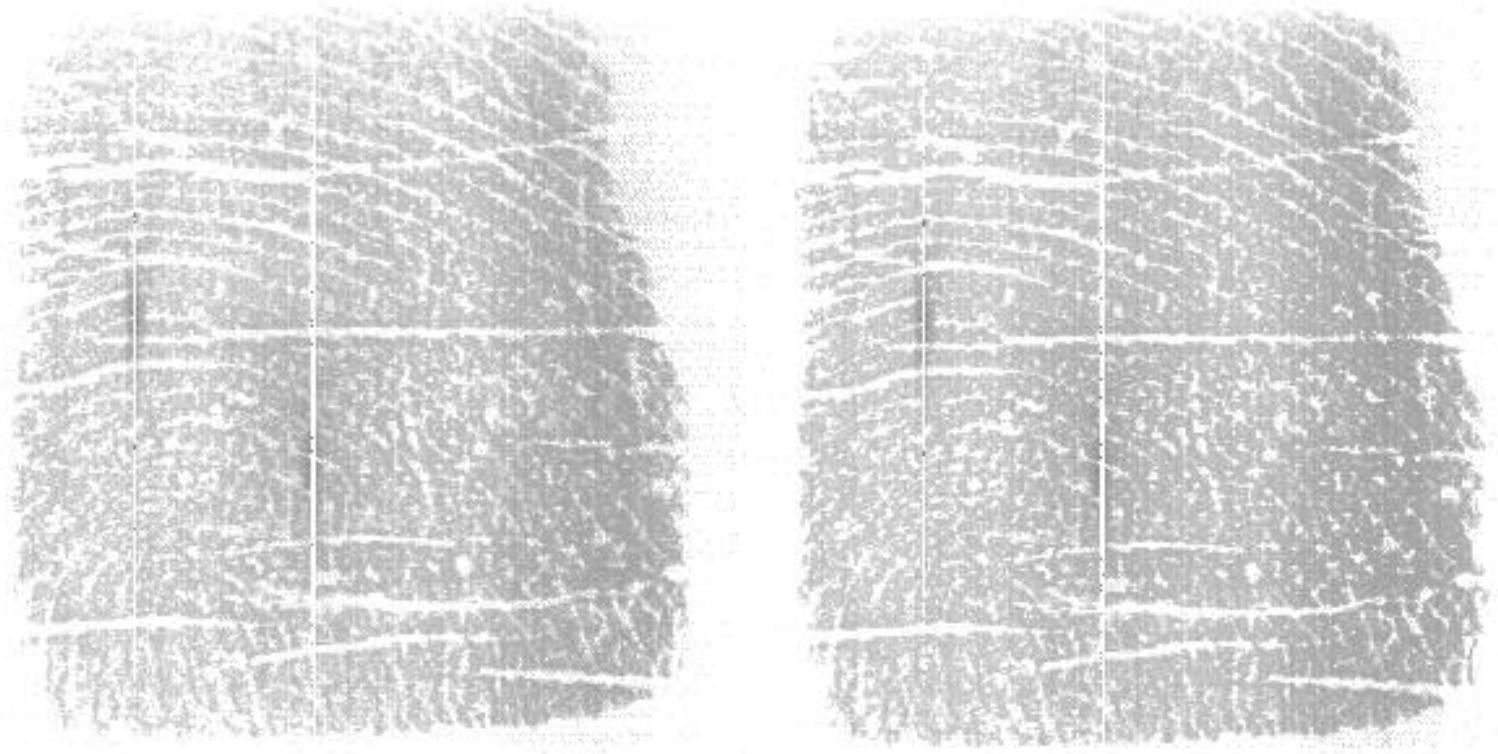

Figure 2-5 The first (left) and last (right) scan of a cadaver fingerprint.
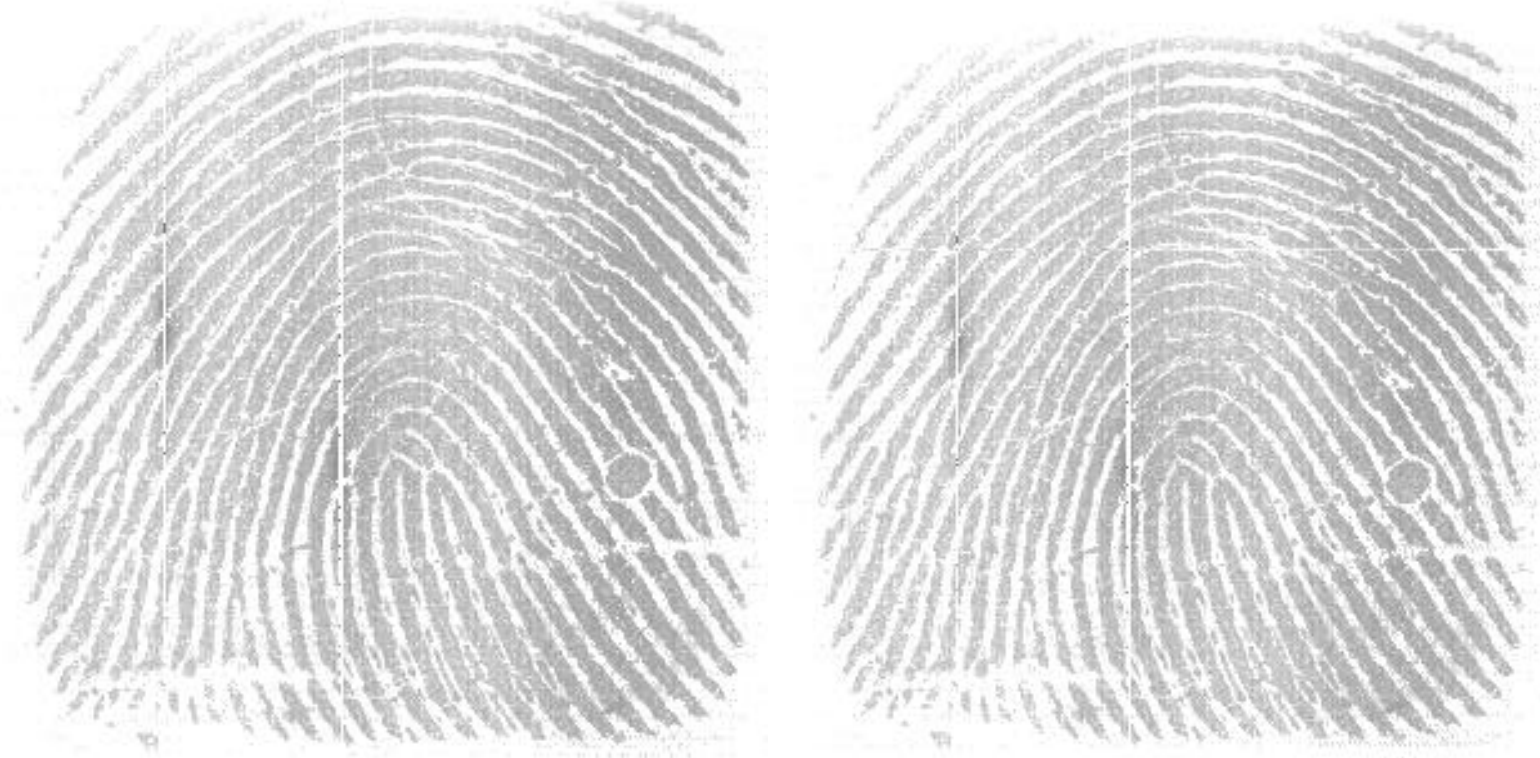

Figure 2-6 The first (left) and last (right) scan of a spoof fingerprint. 
The basis for our method is simple and straightforward. Live fingers, as opposed to cadaver or spoof, demonstrate a temporal change in moisture due to perspiration, and the fingerprint scanner is sensitive to this moisture. The challenge of an image processing algorithm is to quantify the sweating pattern. Furthermore, since this is a physiological phenomenon, this pattern will be variable across subjects, and will also depend on the initial moisture content of the skin.

\section{2-3 The Algorithm}

To quantify this perspiration phenomenon, the algorithm developed in this thesis maps a 2-dimensional fingerprint image to a "signal" which represents the gray level values along the ridges. The last image collected is used to determine the location of the ridges, since it usually has darker ridges and yields better quality. Variations in gray levels in the signal correspond to variations in moisture both statically (on one image) and dynamically (difference between first and last image). A Fourier transform of the signal is used to quantify the "static" variability in gray level along the ridges due to the pores and presence of perspiration. In particular, the algorithm focused on frequencies corresponding to the spatial frequency of the pores. Secondly, dynamic features quantify the change in the local maximums and minimums in the ridge signal.

\section{2-3-1 Overview}

Below are the basic steps performed in the algorithm. The next section will give a detailed description of each step.

1- Capture nine consecutive fingerprints in five seconds. (For the algorithm described in this document, only the first and the last captures are used) 
2- Process the fingerprints to remove noise and device defects, using a noise reduction routine and median filter.

3- Obtain the binary version of the last image using cfltr.exe.

4- Thin the binary image so the ridges are only one pixel wide. Shift the result so that the resulting contours pass through the middle of the ridges.

5- Remove the Y connections so contours only consist of individual curves.

6- Erode two pixels from each to eliminate the extremes, and spurs. Throw away curves shorter than 15 pixels.

7- Use the curves obtained from step 6 as a mask, and convert the gray scales along them into signals for both the first and the last capture $\left(\mathrm{C}_{1}, \mathrm{C}_{2}\right)$.

8- Calculate the FFT of each signal from step 7 and average them for each fingerprint. Calculate the total energy that corresponds to the spatial frequency of the pores. This measure is a static feature.

9- Connect the signals obtained in 7 for both the first and last captures and form a long signal which represents each fingerprint.

10- Detect the local maximums and minimums of first and last fingerprint signals.

11-Calculate a series of parameters (described later) quantifying the sweating process. These measures are dynamic features.

12-Record the results and process the selected features.

13- Make a decision on vitality according to the results of the above process.

The basis of this approach is that after step 9, each fingerprint is transformed into a long signal, whose amplitude is proportional to the level of moisture along the traversed ridges, enabling us to use signal processing and one-dimensional techniques. The flowchart of this algorithm can be found at Appendix E.

\section{2-3-2 Detailed Description}

In this section, each step is described in more detail with related figures.

1. Capture nine consecutive fingerprints: An important point here is that the finger 
should not be moisture-saturated initially. The basis for this algorithm is detection of perspiration. If the skin is already very moist, the scanned will be detected as a temporally stable fingerprint. If the finger is moisture-laden, one can rub his/her finger against a piece of cloth, before the capturing begins. This method was successful for all the scans used during this survey. The sequence of images is produced by issuing 9 consecutive dgrabusb commands with the following settings: dtime 3, dcurr 50 (for discharge time and current). These are the default initial settings for the scanner. Each capture/save takes about 0.5 second, so the last and the first capture are nearly 5 seconds apart. During these 5 seconds, perspiration occurs. Since the device is very sensitive to the developing perspiration, there is a difference in the images between the first and the last scans. The finger should remain still during this 5-second period.

2. Process the fingerprints to remove noise and device defects: Due to the rigors of experimenting with different materials, our scanner had several defects in addition to the usual noise. A Matlab program was developed to clean up the image. It subtracts the permanent defects by comparing it to a "blank" capture taken for each individual case. It also removes the background static by discarding those pixels that change only within $2 \%$ of the "blank" scan. Here are the "before" and "blank" scans with the resultant "after":
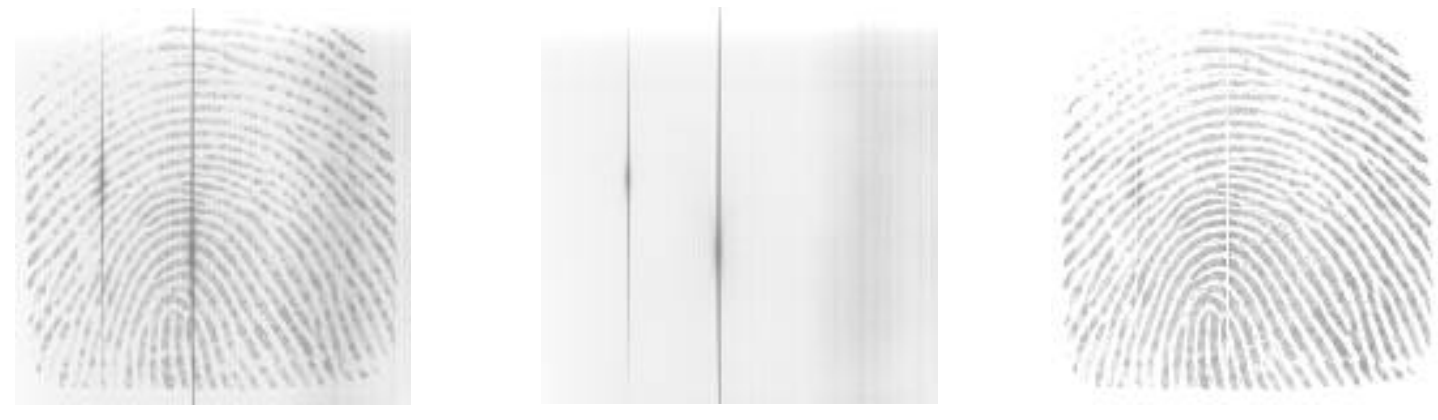

Figure 2-7 Original scan (left), blank scan (center) and scan after noise and defects removed (right).

Next, a 3x3 median filter is applied here to "cover" the white pixels in the middle of the pores. The variations in the pores we want to measure are due to moisture around 
the pore and not the pore itself. This step also smoothes the image further and eliminates "salt \& pepper" noise, if any.

3. Obtain the binary version of the image: Next the program cfltr transforms the image to a binary image (Figure 2-8).
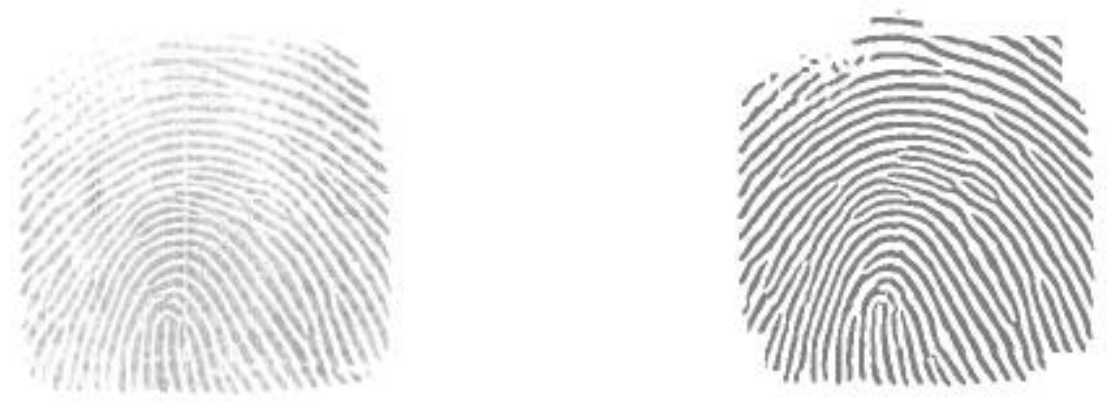

Figure 2-8 Image after step 2 (left), step 3 (right)

4,5,6. Thin, remove $Y$ connections, erode: Thinning the binary images finds the location of the fingerprint ridges. However, since the result does not pass through the middle of the original ridges, a shift is necessary. Y-junctions are removed using a simple $3 \times 3$ non-overlapping neighbor operation. The results of the these three steps can be seen in Figure 2-9, where the extracted curves are superimposed on the original fingerprint for visualization.

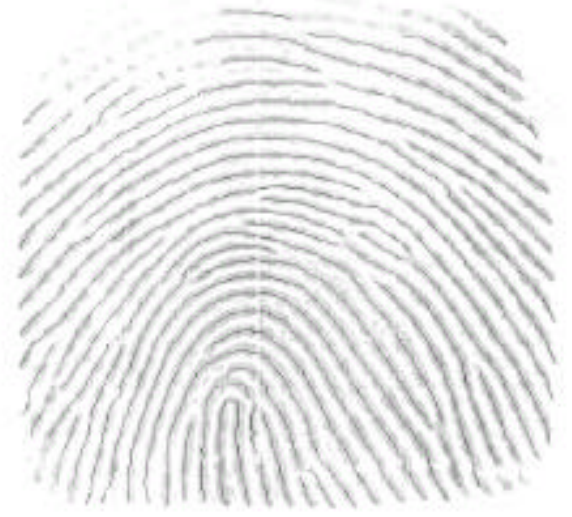

Figure 2-9 Fingerprint ridges as found by steps 4, 5, and 6 overlaid on the fingerprint image

Note that the $\mathrm{Y}$ connections of Figure 2-8 no longer exist. The 2-pixel erosion also eliminates the extremes of the ridges and accidental spurs. Curves shorter than 15 
pixels are discarded since the nominal pore-to-pore distance is around $0.5 \mathrm{~mm}$, spanning almost 10 pixels (though this distance can vary for different people and different regions on the fingertip).

7, 8-Mask and build the strings, take FFT: The curves which traverse through the middle of the ridges (Figure 2-9) have varying gray levels in the fingerprint image. The peaks denote the moist (pore) locations and the valleys show the dryer regions, usually between each two pores. (This is further described in 9, 10, and 11.) As can be seen from Figure 2-2 and Figure 2-14, there is regularity related to the spacing of the pores in the live fingerprint signal. The peak-to-peak distance is around 10 pixels $(0.5 \mathrm{~mm})$ which is in accord with pore-to-pore distance. The variations in the cadaver/spoof fingerprint signal do not correspond to a specific periodicity because they do not have evenly spaced perspiring pores (Figures 2-15, 2-16).

The main feature, which quantifies this, is the average Fourier transform of the signal segments where the energy related to the typical pore spacing is used. A 256point FFT command is performed in Matlab. Total energy is evaluated for a 8-24 pixel distance (for a pore spacing of 0.4 to $1.2 \mathrm{~mm}$ ) which takes into account the case of one missing pore for maximum spacing of $0.6 \mathrm{~mm}$. This corresponds to a spatial frequency range is between 11 and 33 (\# of FFT points / spatial period). Before taking the FFT, in order to eliminate the spike around zero frequency, the DC of the signal (only for this specific calculation) was removed. The above procedure can be mathematically expressed as:

$$
S M=\sum_{k=11}^{33} f(k)^{2} \quad \text { (Static Measure) }
$$

where 

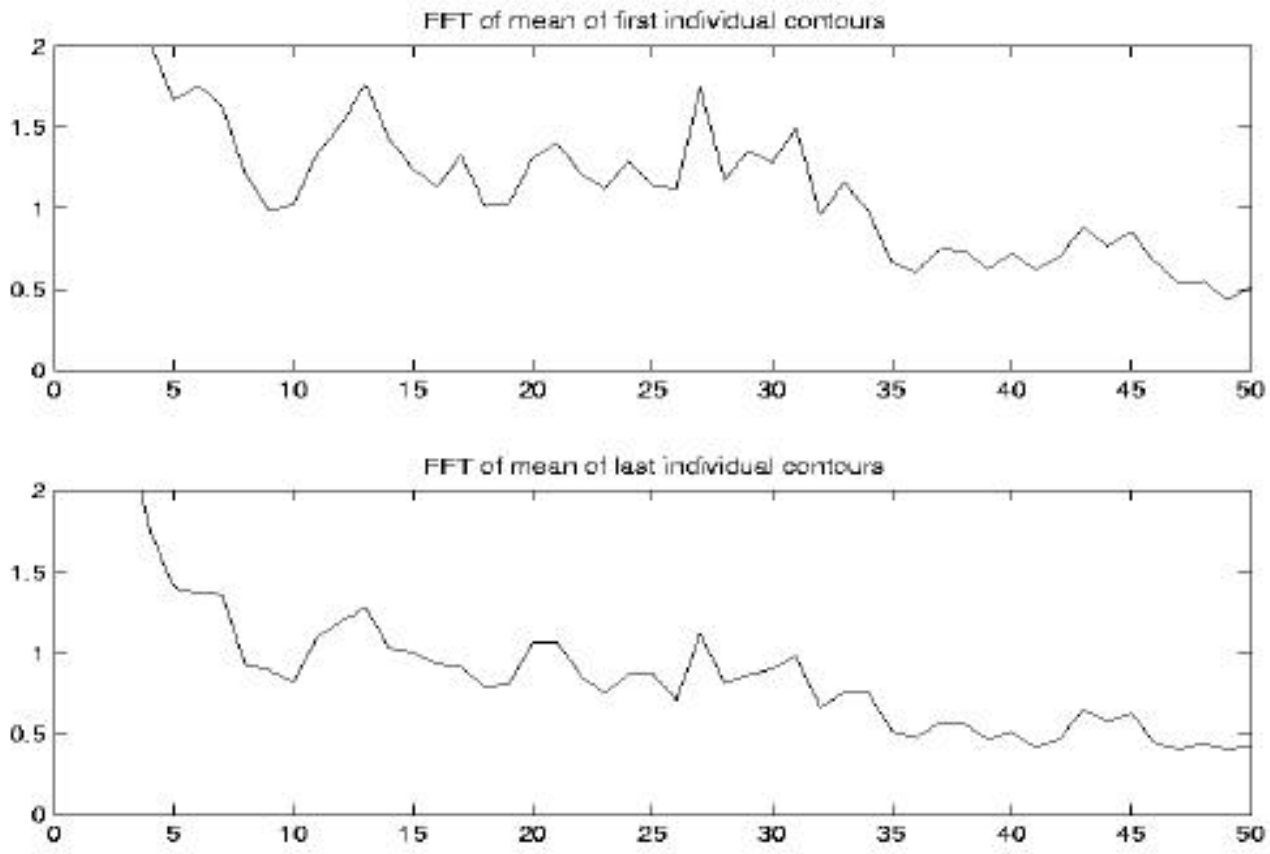

Figure 2-10 The average of the FFTs calculated from signal segments from the first (top) and last (bottom) capture from the live fingerprint shown in Figure 2-19.

$f(k)=\frac{\sum_{i=1}^{n}\left|\sum_{j=1}^{256} S_{l i}^{a} e^{-j 2 \Pi(k-1)(i-l) / 256}\right|}{n} \quad, \quad S_{l i}^{a}=S_{l i}-\operatorname{mean}\left(S_{l i}\right)$

$\mathrm{n}$ is number of individual strings $\left(\mathrm{S}_{\mathrm{i}}\right)$ in the processed fingerprint mask from last capture obtained in step 6 (\# of 8 connected objects longer than 15 pixels) so that

$$
\sum_{i=1}^{n} S_{1 i}(j)=C_{1}
$$

As will be seen in the results, this proves to be an excellent measure. Figures 2-10, 211, and 2-12 give the average FFT for the first and last images for live, cadaver, and spoof fingerprints respectively. The energy for cadaver and spoof is very low compared to live. In addition, the energy for the last live fingerprint is smaller relative to the first capture. This is logical, since the swing of the signal decreases in time as the moisture is spread more evenly. The results of static measure for live, cadaver, and spoof fingerprints are depicted in Figures 3-1 to 3-4. 

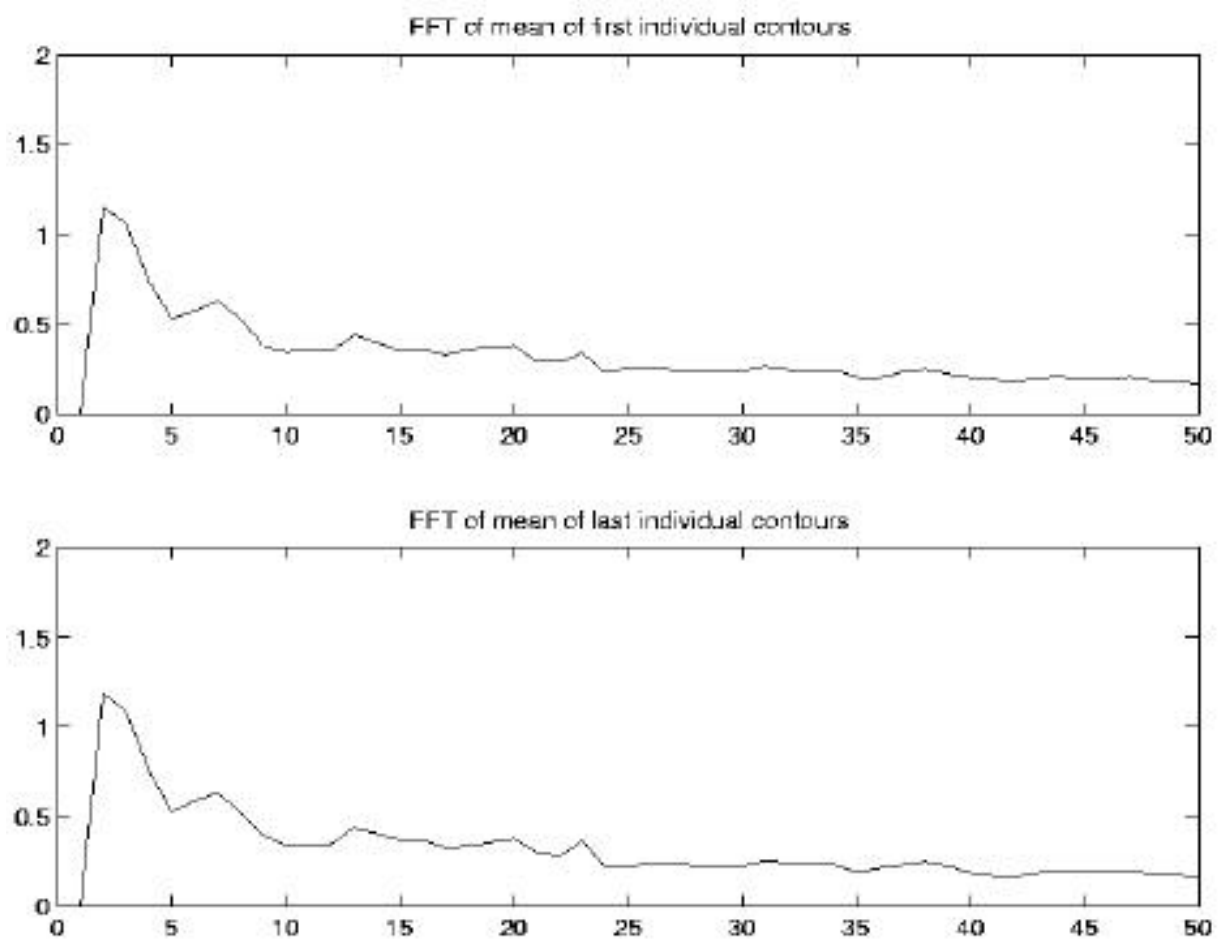

Figure 2-11 Same as above for a fingerprint from a cadaver.
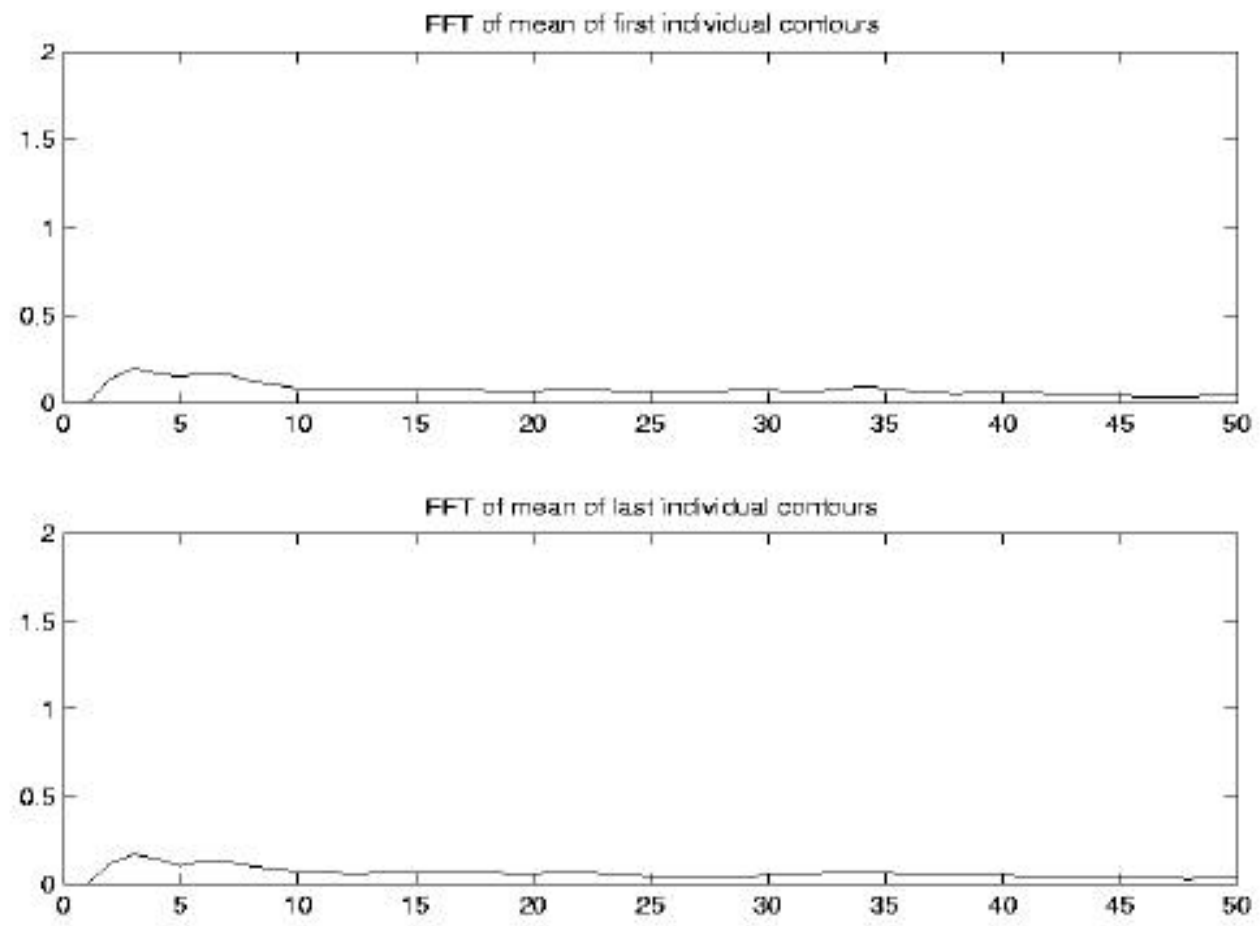

Figure 2-12 Same as above for a spoof fingerprint. 
One problem with the static measure is that of the grainy texture the play dough spoof finger (Figure 2-12). It introduces many unwanted frequency components. The dynamic measures attempt to quantify the temporal changes of the "fingerprint signal" to assist the differentiation between spoof/cadaver and live.

9- Connect strings into fingerprint "signals": Individual strings are connected to form a long signal, which describes the gray levels of the contours passing through middle of the ridges (Figure 2-13).

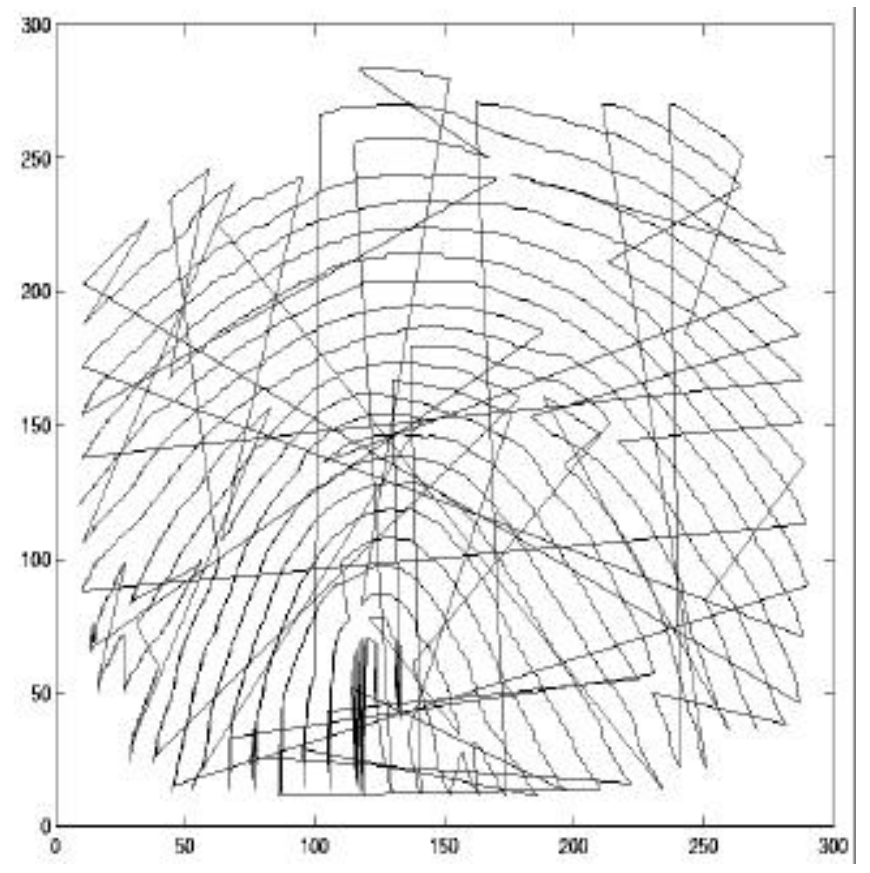

Figure 2-13 Connected contours.

Figures 2-14, 2-15, and 2-16 are three (magnified) samples from portions of the signals extracted from a live, cadaver, and spoof fingerprint, respectively. 


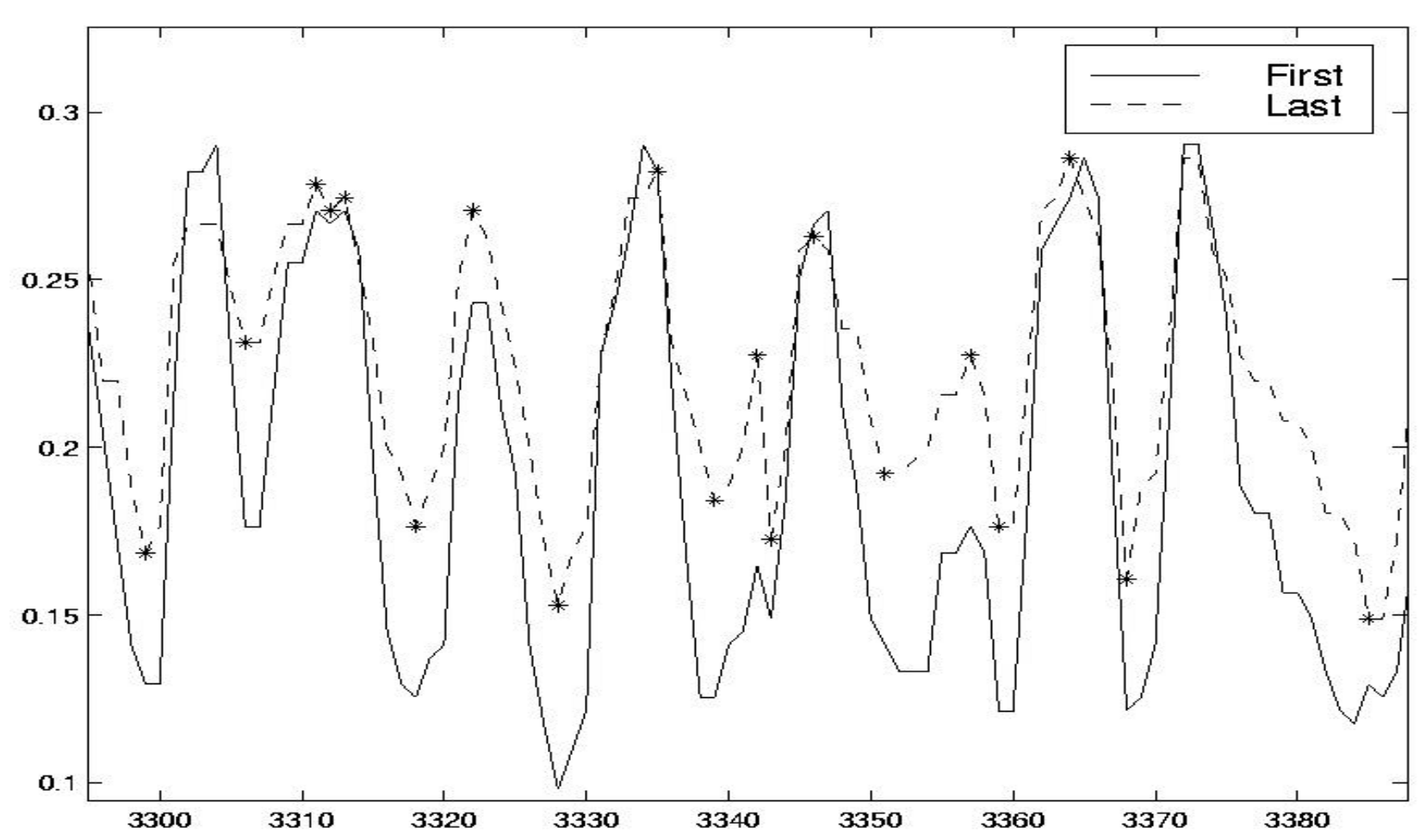

Figure 2-14 Portion of a live fingerprint signal. * denotes minimums and maximums.

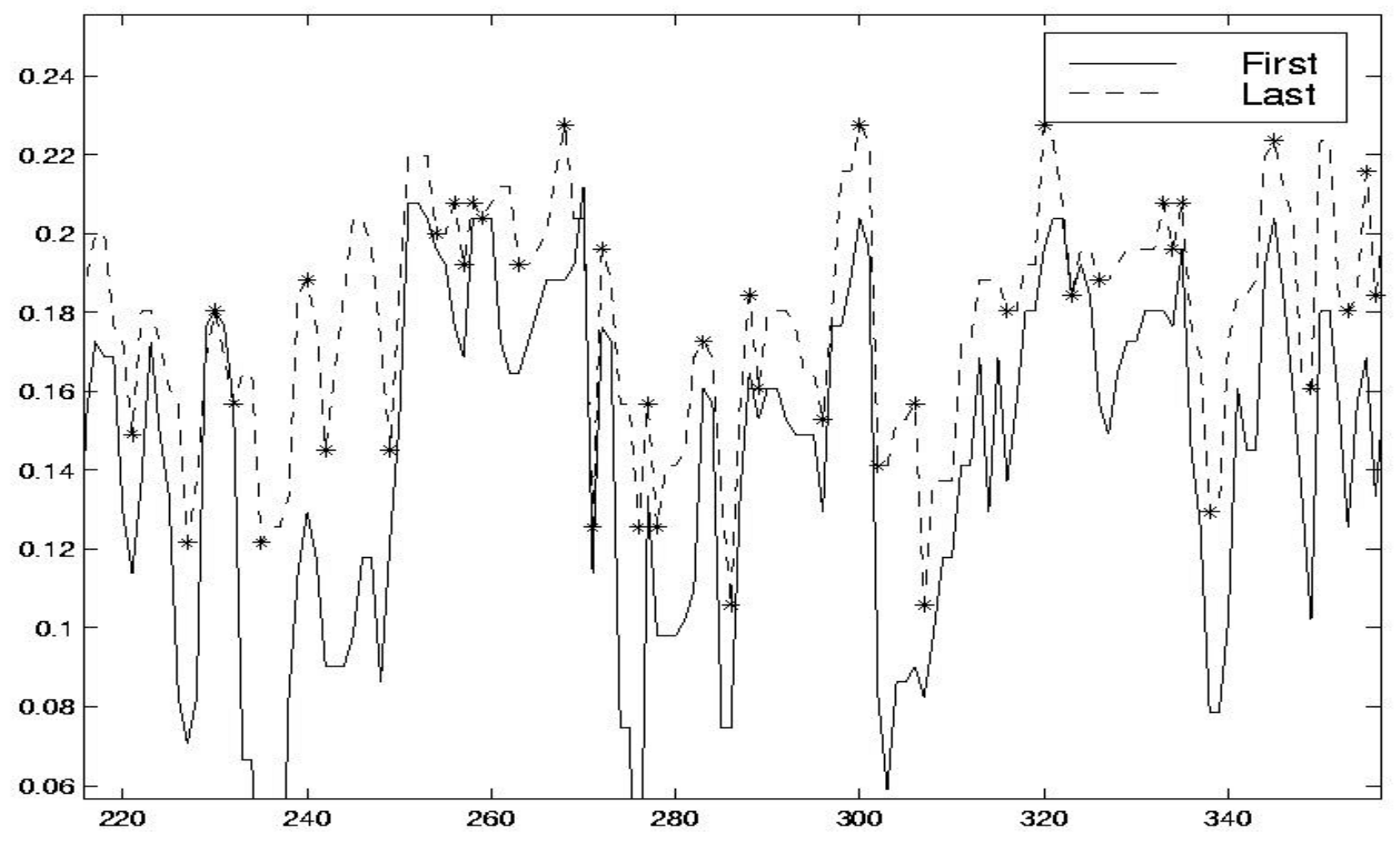

Figure 2-15 Portion of a cadaver fingerprint signal. * denotes minimums and maximums. 


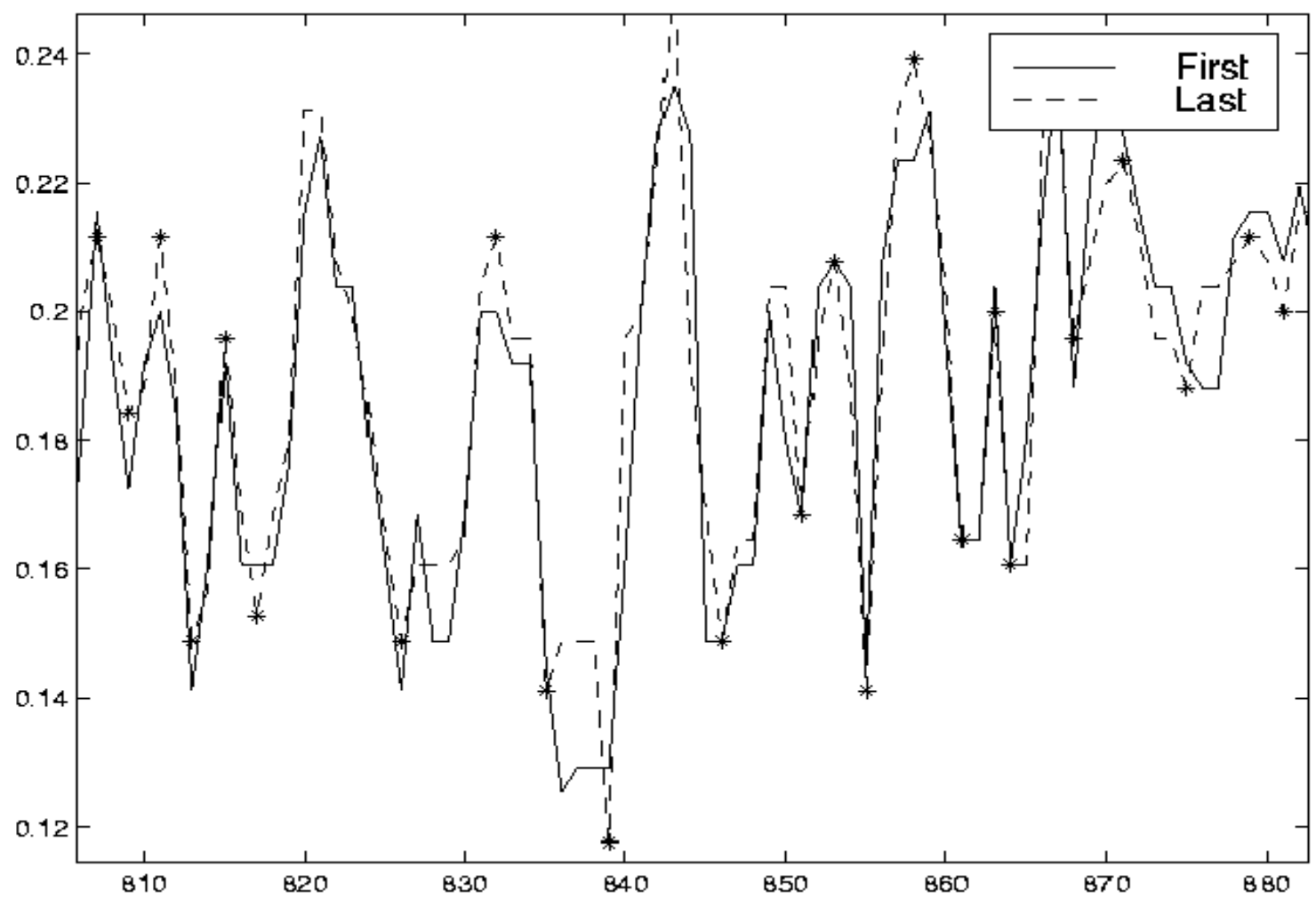

Figure 2-16 Portion of a spoof fingerprint signal, * denotes maximums and minimums.

10, 11- Dynamic features: The dynamic features are described below:

The general swing (local maximum minus local minimum) for the live fingerprint is usually larger than that of the spoof and cadaver. In addition, this swing is typically smaller for the last capture compared to first. It is hypothesized that starting with a dry live finger, moisture begins mainly around the pores creating peaks in the signal. Gradually the moisture spreads along the ridges and the total swing decreases in time. This trend is not present for spoof and cadaver fingerprint signals. However, it should be noted, there is a general darkening effect over time for the captured fingerprints by the device, especially for the cadaver fingers.

For live fingerprint signals, the maximums are fairly constant, but the minimums increase from first to last capture. It is hypothesized that the pixels near the pores are relatively saturated with perspiration while areas between the pores are still relatively 
dry. The only exception is when in the finger is extremely dry so even the pore area is not saturated. Based on the above, four dynamic features were introduced in the next section.

- Total swing ratio of first to last fingerprint signal: According to our hypothesis, the fluctuation of the live fingerprint signal should be more in the first capture when we have moist pores and drier regions in between the pores (and so higher peaks and lower valleys) compared to last fingerprint signal where the sweat has diffused into drier regions (and there are less variations in gray level). This effect can be seen in Figures 3-5 through 3-8. The average absolute value of the signal swing used, because fingerprint signal lengths differ for different cases (Dynamic measure 1). In mathematical terms, the first dynamic measure (DM1) is as follows:

$$
D M I=\frac{\sum_{i=1}^{m}\left|C_{1 i}-C_{1 i-1}\right|}{\sum_{i=1}^{m}\left|C_{2 i}-C_{2 i-1}\right|}
$$

$\mathrm{C}_{1 \mathrm{i}}$ is referring to the gray level signal points the ridges of the first capture. $\mathrm{C}_{2 \mathrm{i}}$ is the same except for that it is for the second capture. $i$ is equal to 1 to the length of the ridge signal (m). It is the same for $\mathrm{C}_{1}$ and $\mathrm{C}_{2}$ (since the same mask was used for $\mathrm{C}_{1}$ and $\mathrm{C}_{2}$ ).

- Min/Max growth ratio of first to last fingerprint signal: For the live fingerprint signal, the height of the maximums doesn't increase as fast as the minimums. So the average ratio of the maximum growth to minimum growth of first compared to last should be larger for the live fingerprint signal compared to cadaver and spoof. This can be clearly seen from figures 3-9 through 3-12. In mathematical terms, dynamic measure 2 (DM2) is as follows: 


$$
D M 2=\frac{\sum_{j}\left(C_{2 j}^{\min }-C_{l j}^{\min }\right)}{\sum_{k}\left(C_{2 k}^{\max }-C_{l k}^{\max }\right)}
$$

$\mathrm{C}_{1 \mathrm{j}}{ }^{\mathrm{min}}$ and $\mathrm{C}_{2 \mathrm{j}}{ }^{\mathrm{min}}$ are the local minimums for the first and last scan, respectively. Minimums where determined from the second scan and applied to first. $j$ is the index for each minimum in the ridge signal. $\mathrm{C}_{1 \mathrm{k}}{ }^{\min }, \mathrm{C}_{2 \mathrm{k}}{ }^{\min }$, and $\mathrm{k}$ are same as above except that they are for local minimums.

- Last-first fingerprint signal difference mean: When the first ridge signal $\left(\mathrm{C}_{1}\right)$ is subtracted from the last $\left(\mathrm{C}_{2}\right)$, the difference for a finger with no life less than a finger that is perspiring quantifying a temporal pattern of moisture. Though there is a general darkening effect for cadaver fingers over time, but it translates to a signal with baseline shifting up while maintaining the same ac, especially for spoofs. This baseline shift cancels out in the differencing procedure. Figures 3-13 through 3-16 demonstrate these changes. In mathematical terms, dynamic measure 3 (DM3) is as follows:

$$
D M 3=\frac{\sum_{i=1}^{m}\left(C_{2 i}-C_{l i}\right)}{m}
$$

$\mathrm{m}, \mathrm{C}_{1 \mathrm{i}}$, and $\mathrm{C}_{2 \mathrm{i}}$ are the same as in DM1.

- Percentage change of standard deviations of first and last fingerprint signals: The last proposed measure in the dynamic ensemble is the percentage change in standard deviation of last and first fingerprint signals for each case. The rational behind it is similar to the others: if the fluctuation of the ridge signal is decreasing around the mean (the change typical for live fingerprint signal), the fourth dynamic measure (DM4) will increase (Figures 3-17 through 3-20). In mathematical terms, 


$$
D M 4=\frac{S D\left(C_{1}\right)-S D\left(C_{2}\right)}{S D\left(C_{1}\right)}
$$

where SD is the standard deviation operator:

$$
S D\left(C_{1}\right)=\sqrt{\frac{\sum_{i=1}^{m}\left(C_{1 i}-\operatorname{mean}\left(C_{1}\right)\right)^{2}}{m-1}}
$$

In classification, absolute value of DM4 has been used.

12,13- Process the results and make a decision on vitality: Classification can be performed based on only one of the developed measures. However, a decision based on a combination of static and four dynamic measures is able to give much better classification. 
Chapter 3. Results 
The results of each measure are presented using sorted graphs followed by a corresponding relative operating characteristic curve (ROC). The results for each measure across subjects are sorted in ascending order for better visualization. Therefore, the numbering on horizontal axis $(1-18)$ does not correspond to a particular sample. The relative operating characteristic (ROC) curves plot false acceptance ratio (FAR) versus false rejection ratio (FRR) for varying thresholds in order to separate groups. FAR is defined as the percentage of cadaver or spoof fingerprints that are detected as live. FRR is defined as the percentage of live fingerprints that are detected as cadaver/spoof. The results are separated for live vs. spoof samples $(18+18)$, and live vs. cadaver $(18+18)$. Similar plots for each of the five measures are presented. 

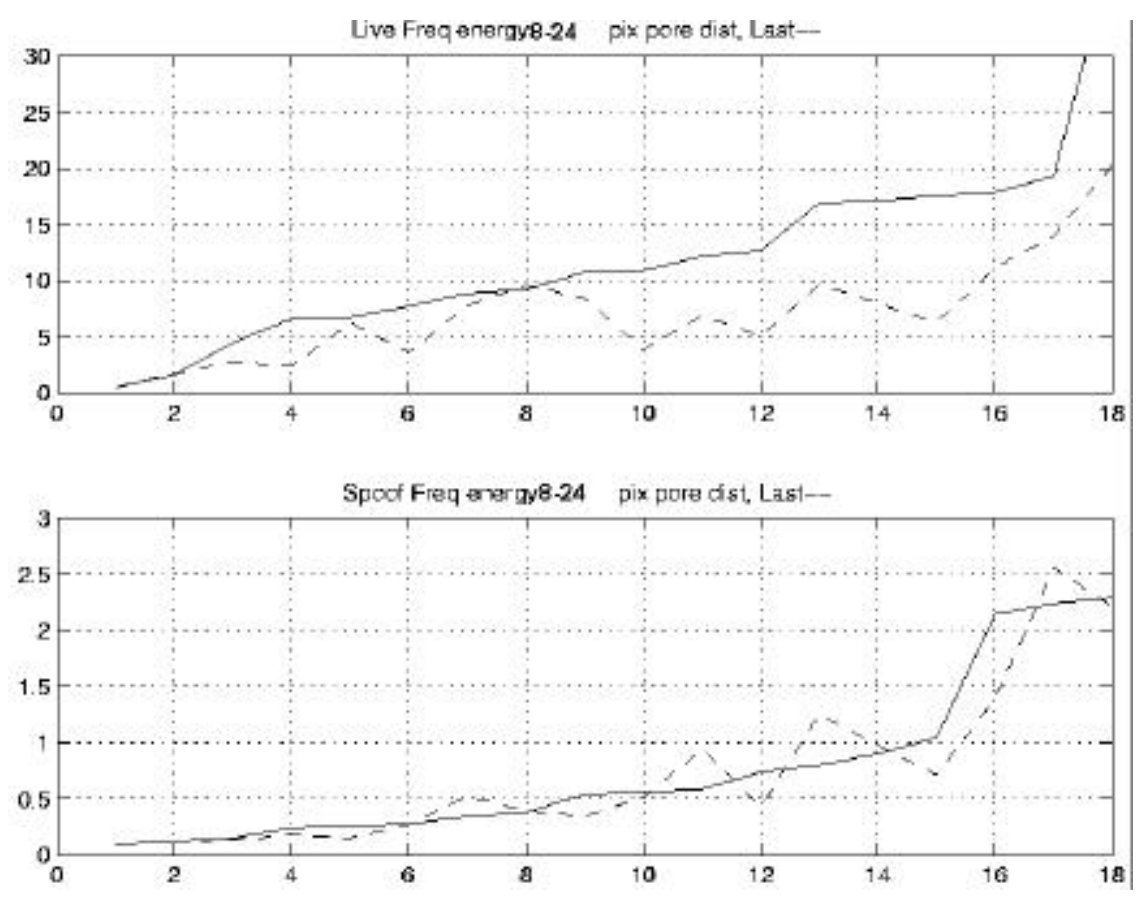

Figure 3-1 Static measure (SM). The energy (y-axis) for live (top) and spoof (bottom) corresponding to pore separation of 8-24 pixels $(0.4-1.2 \mathrm{~mm})$, dashed for last capture and solid for first. Note that the vertical scales are different. The X-axis is "sorted" subjects.

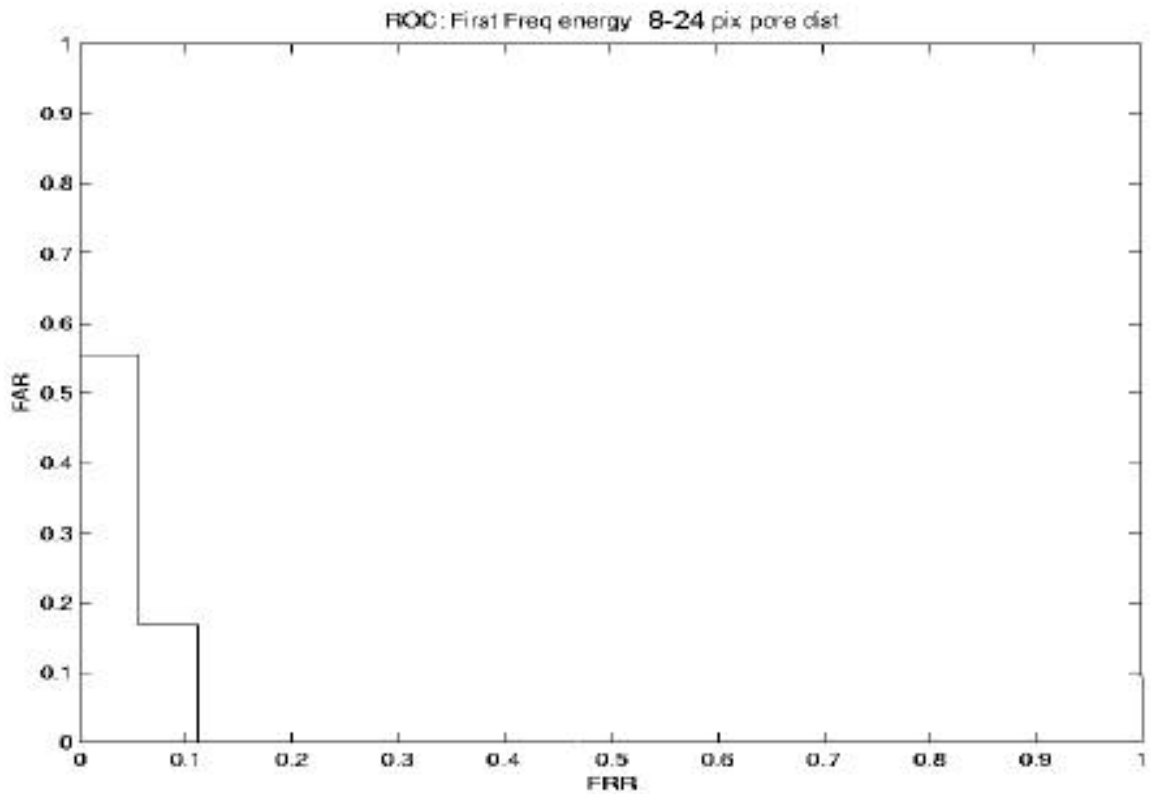

Figure 3-2 ROC associated with Figure 3-1. The line is the FAR and FRR due to threshold change for the static measure. FAR is false acceptance rate of spoof detected as live and FRR is the false rejection rate of live detected as spoof. 

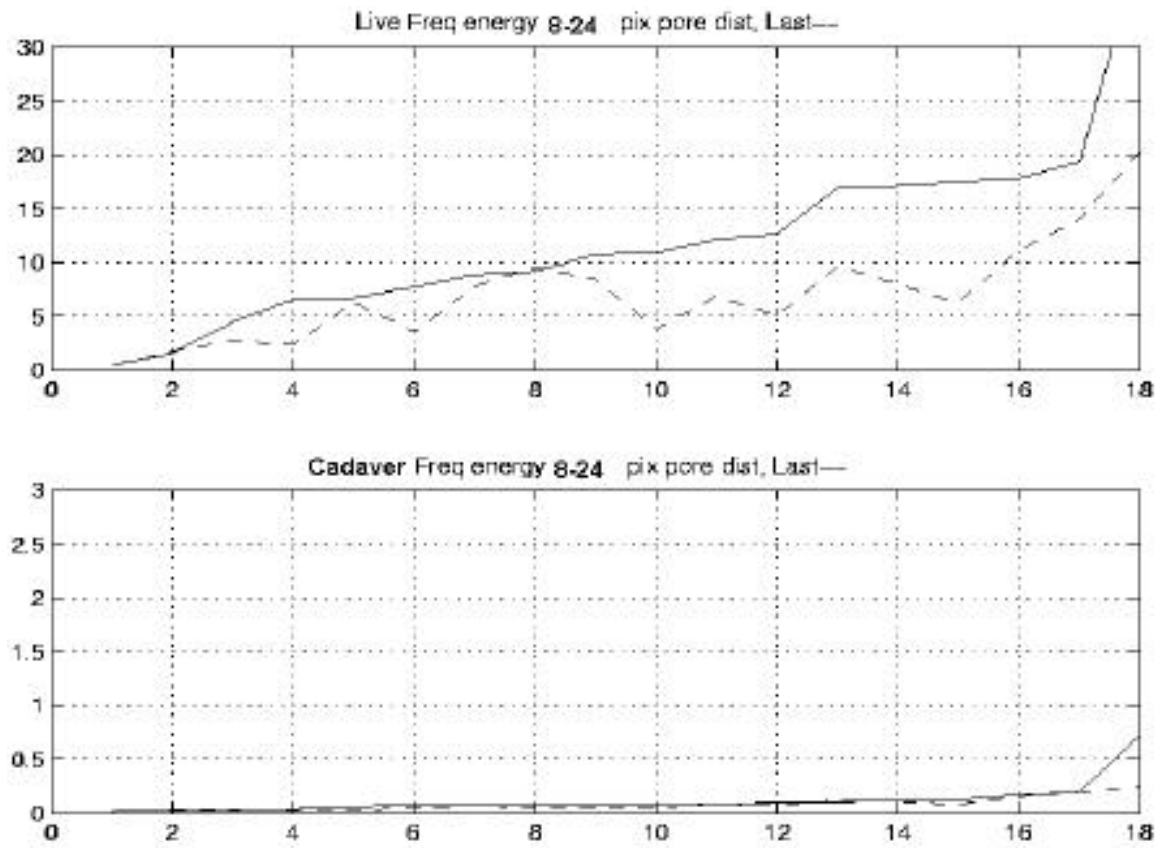

Figure 3-3 Static measure (SM). The energy (y-axis) for live (top) and cadaver (bottom) corresponding to pore separation of 8-24 pixels $(0.4-1.2 \mathrm{~mm})$, dashed for last capture and solid for first. Note that vertical scales are different.



Figure 3-4 The ROC associated with Figure 3-3. The line is the FAR and FRR due to threshold change for the static measure. FAR is false acceptance rate of cadaver detected as live and FRR is the false rejection rate of live detected as cadaver. 


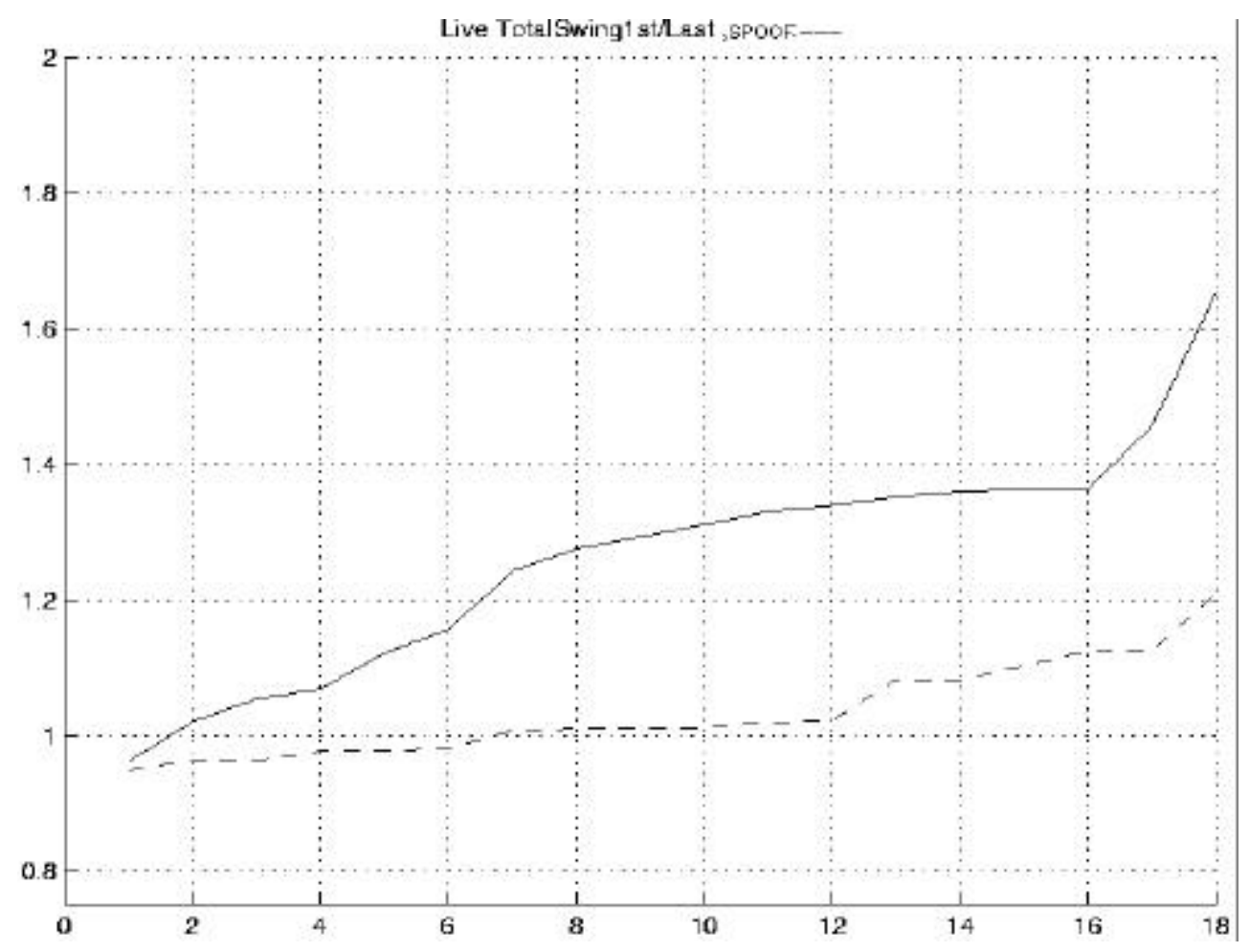

Figure 3-5 First/last total swing ratio (DM1) for live (solid) and spoof (dashed).

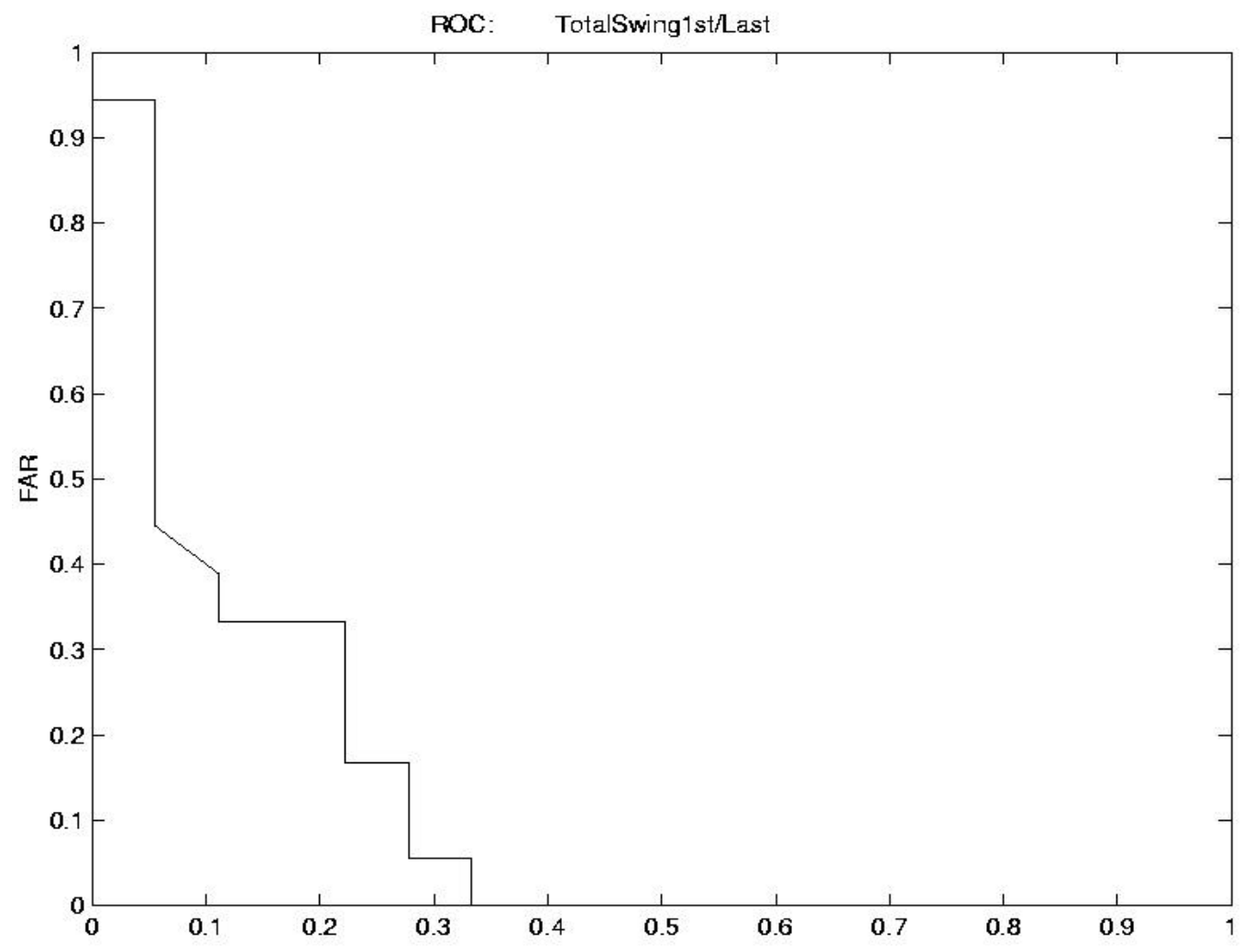

Figure 3-6 The ROC associated with Figure 3-5. 


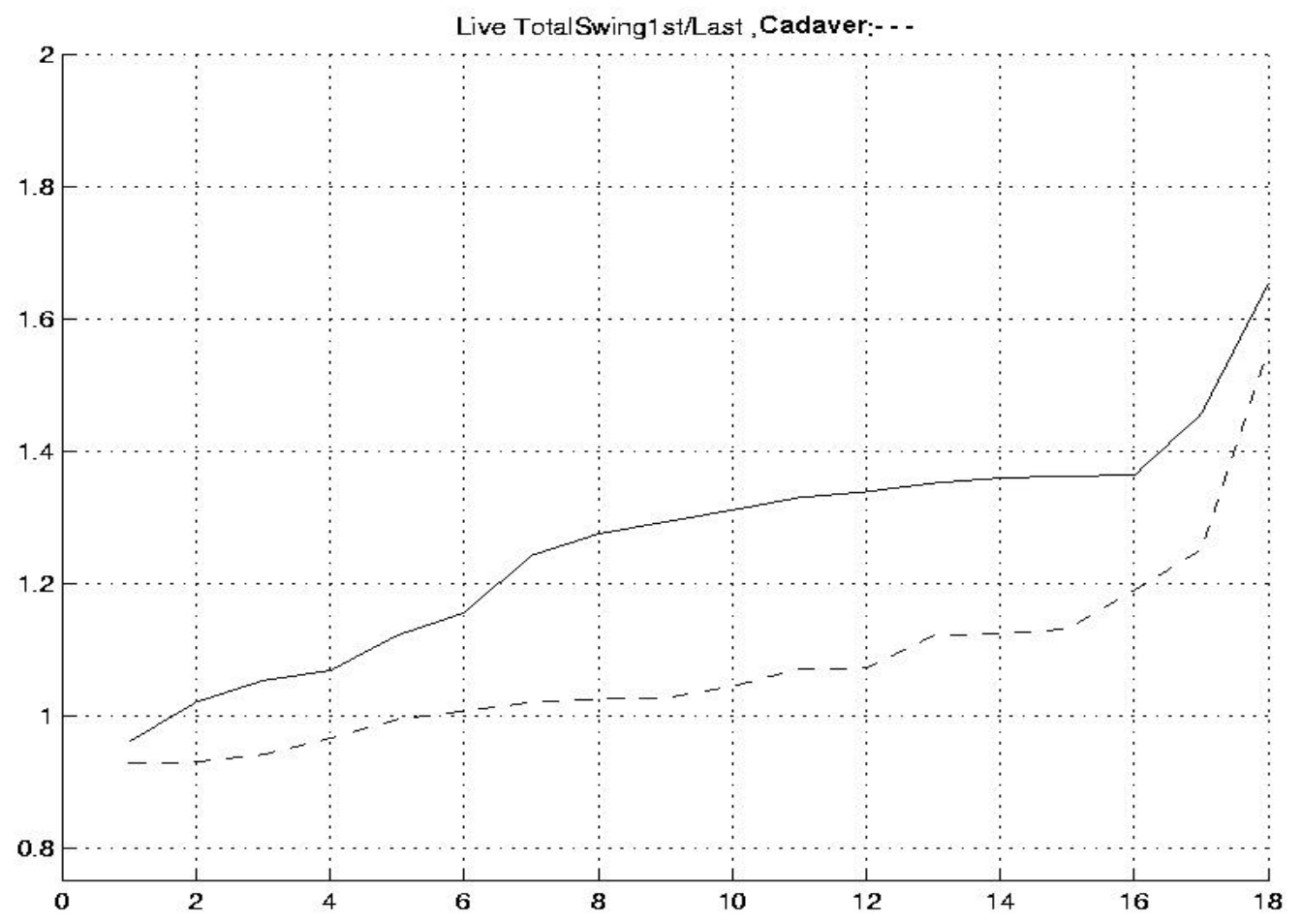

Figure 3-7 First/last total swing ratio (DM1) for live (solid) and cadaver (dashed).

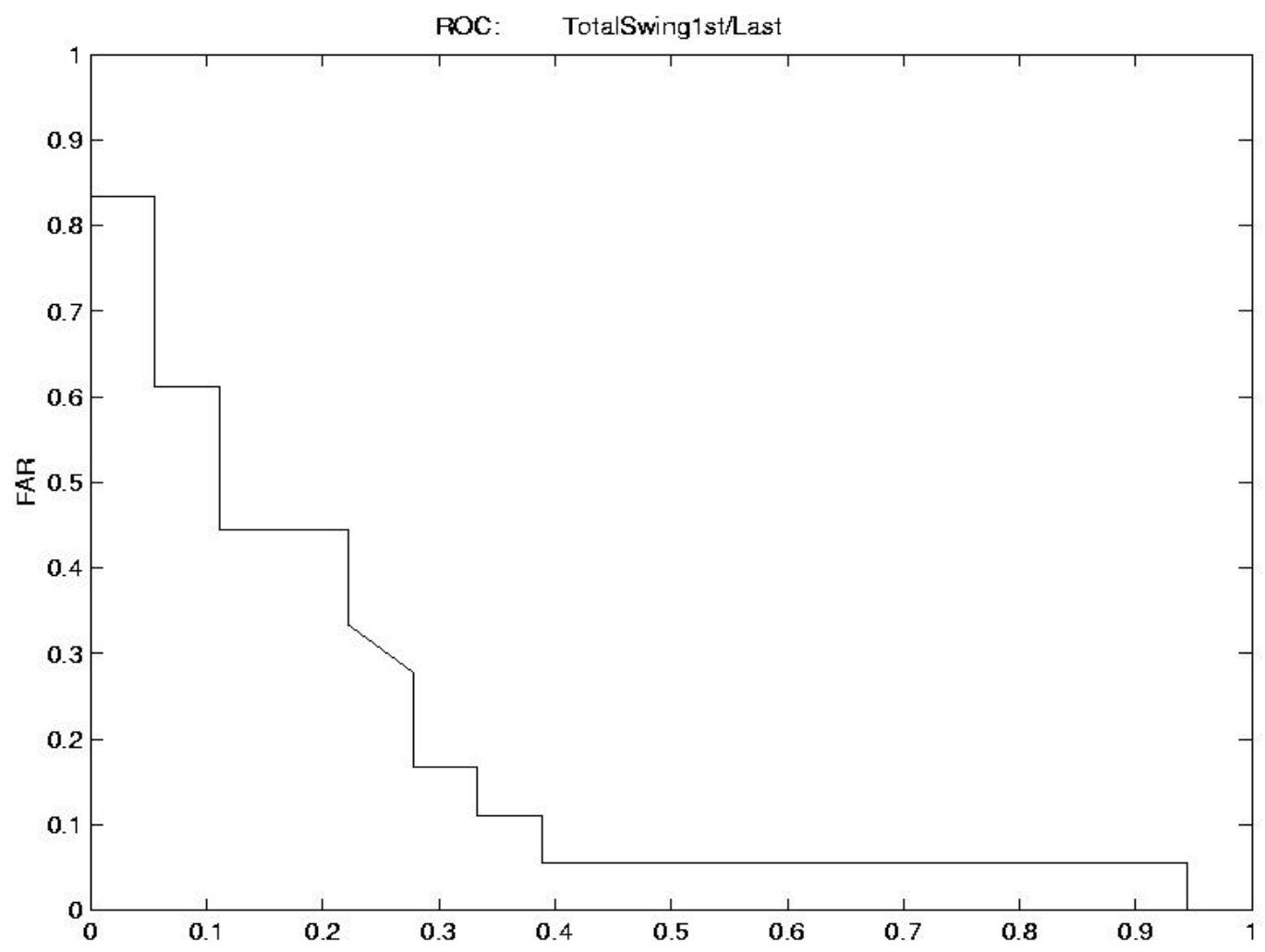

Figure 3-8 The ROC associated with Figure 3-7. 


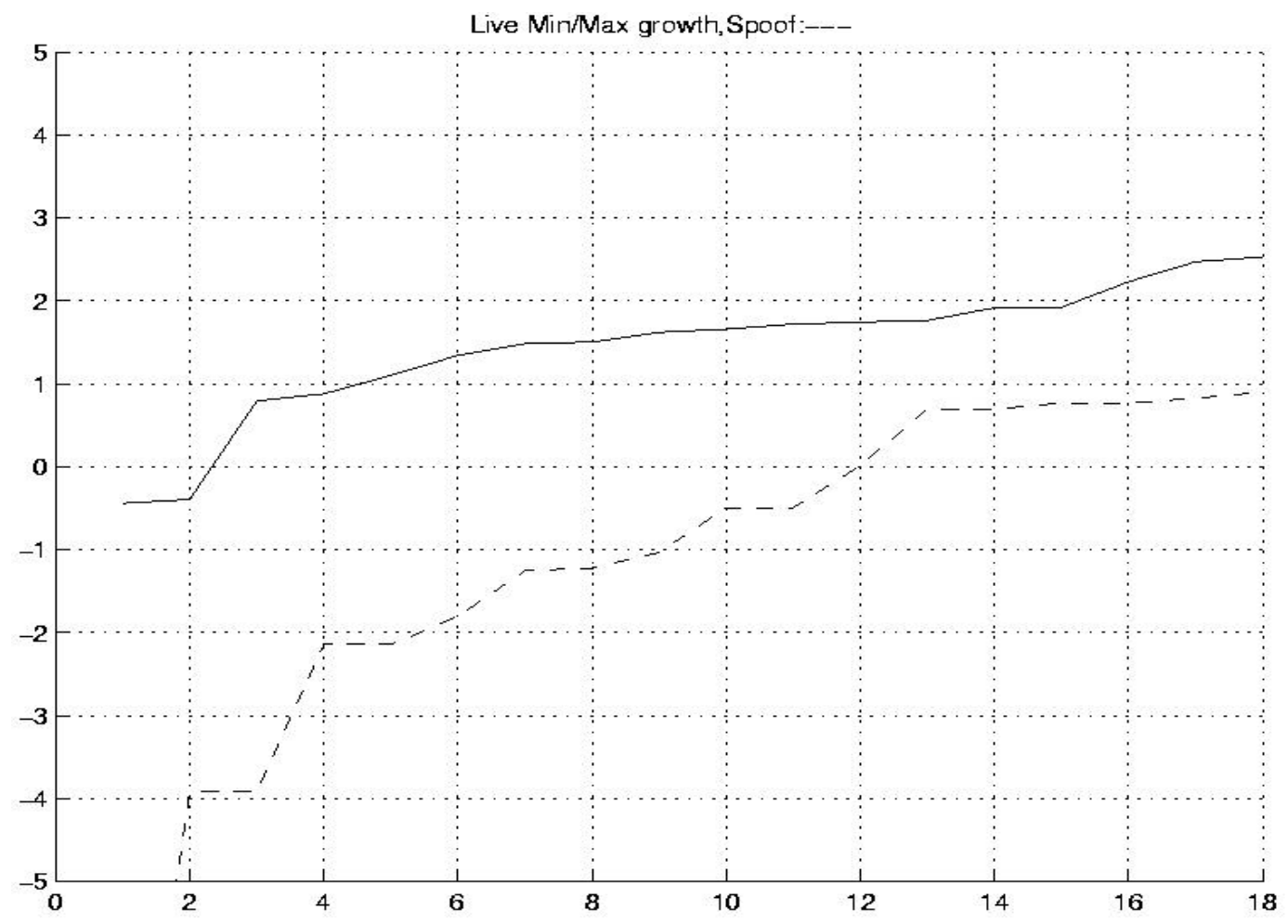

Figure 3-9 Min/max growth ratio (DM2) for live (solid) and spoof (dashed).

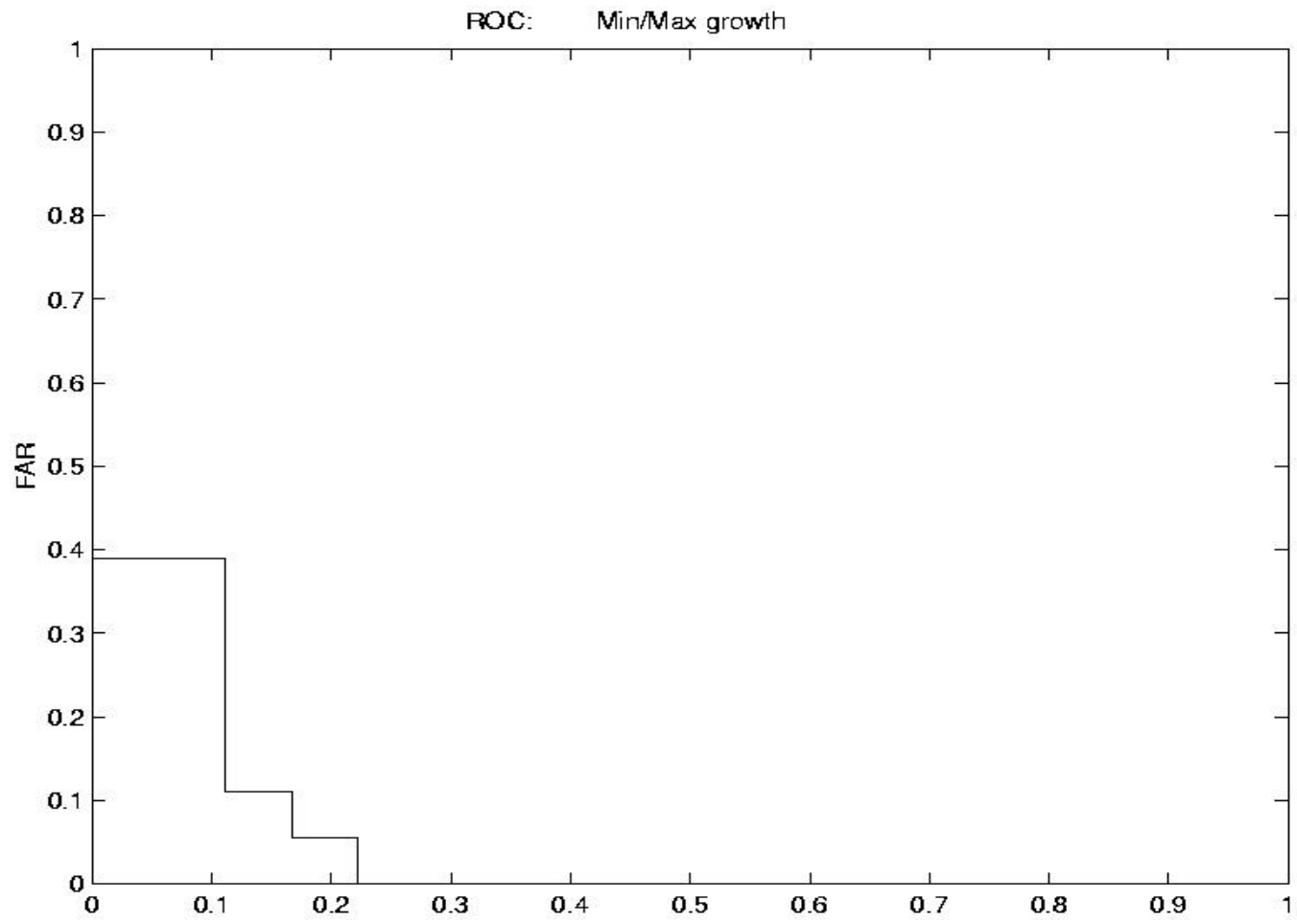

Figure 3-10 The ROC associated with Figure 3-9. 


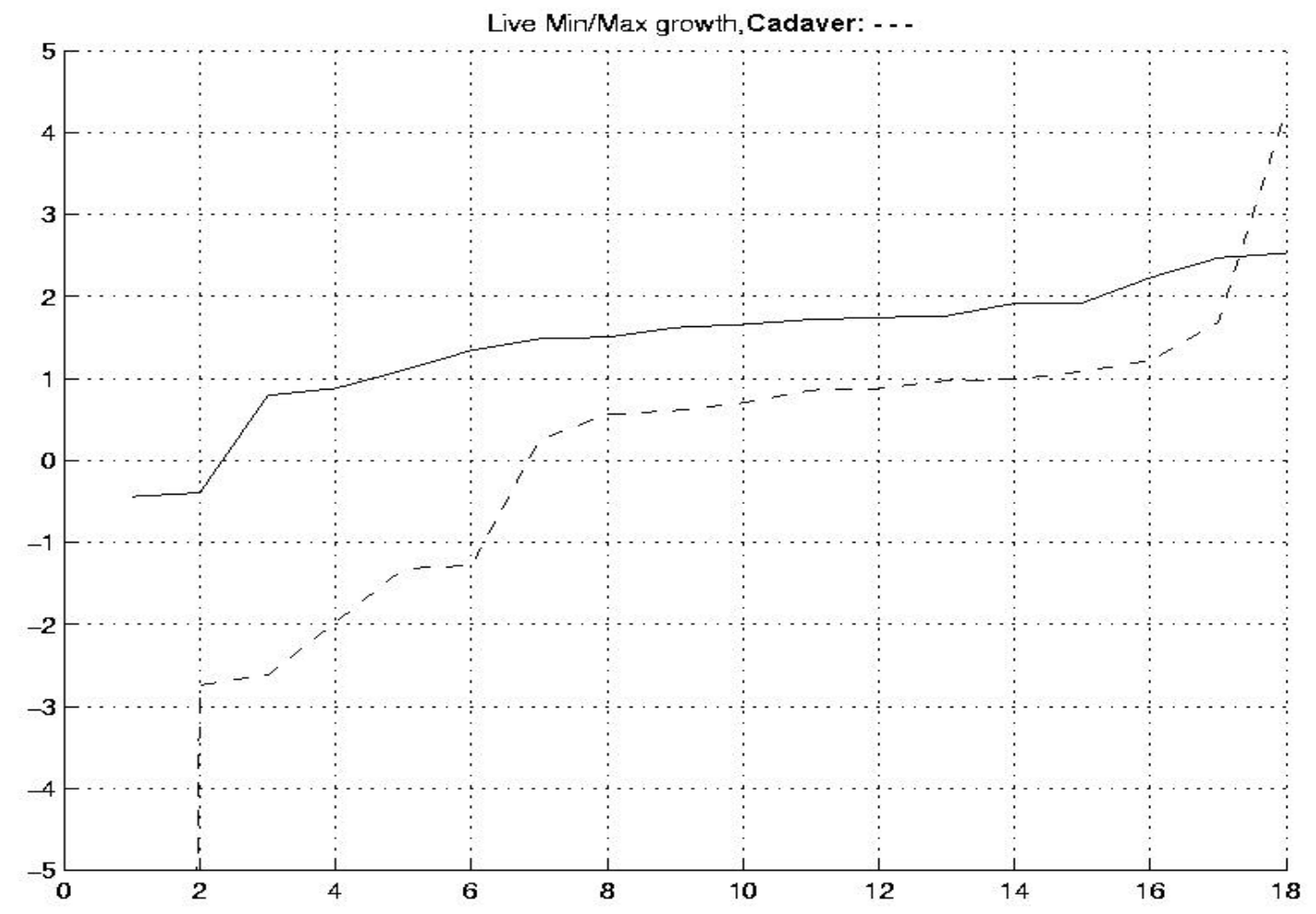

Figure 3-11 Min/max growth ratio (DM2) for live (solid) and cadaver (dashed).

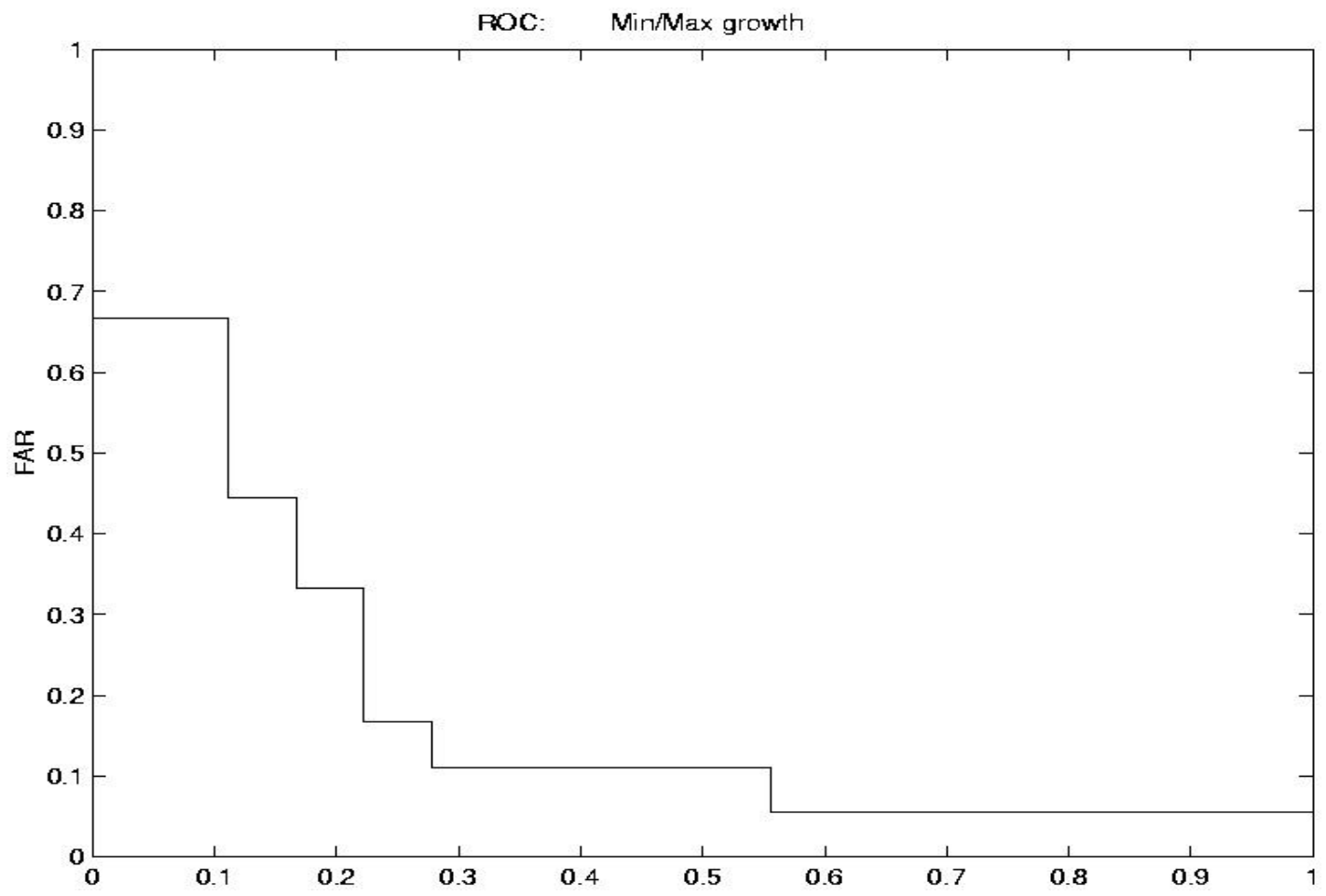

Figure 3-12 The ROC associated with Figure 3-11. 



Figure 3-13 Mean of last minus first signals (DM3) for live (top) and spoof (bottom).

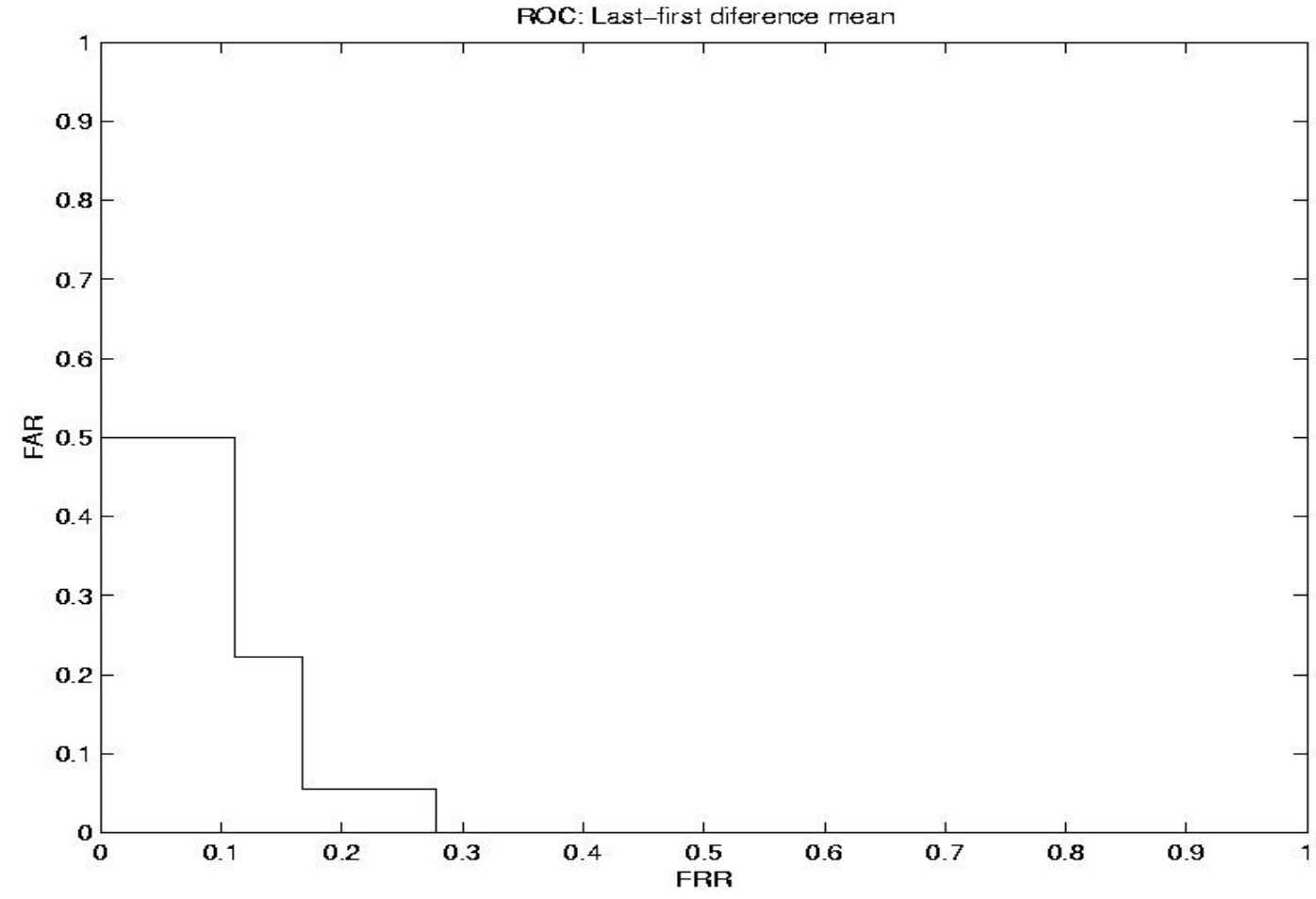

Figure 3-14 The ROC associated with Figure 3-13. 

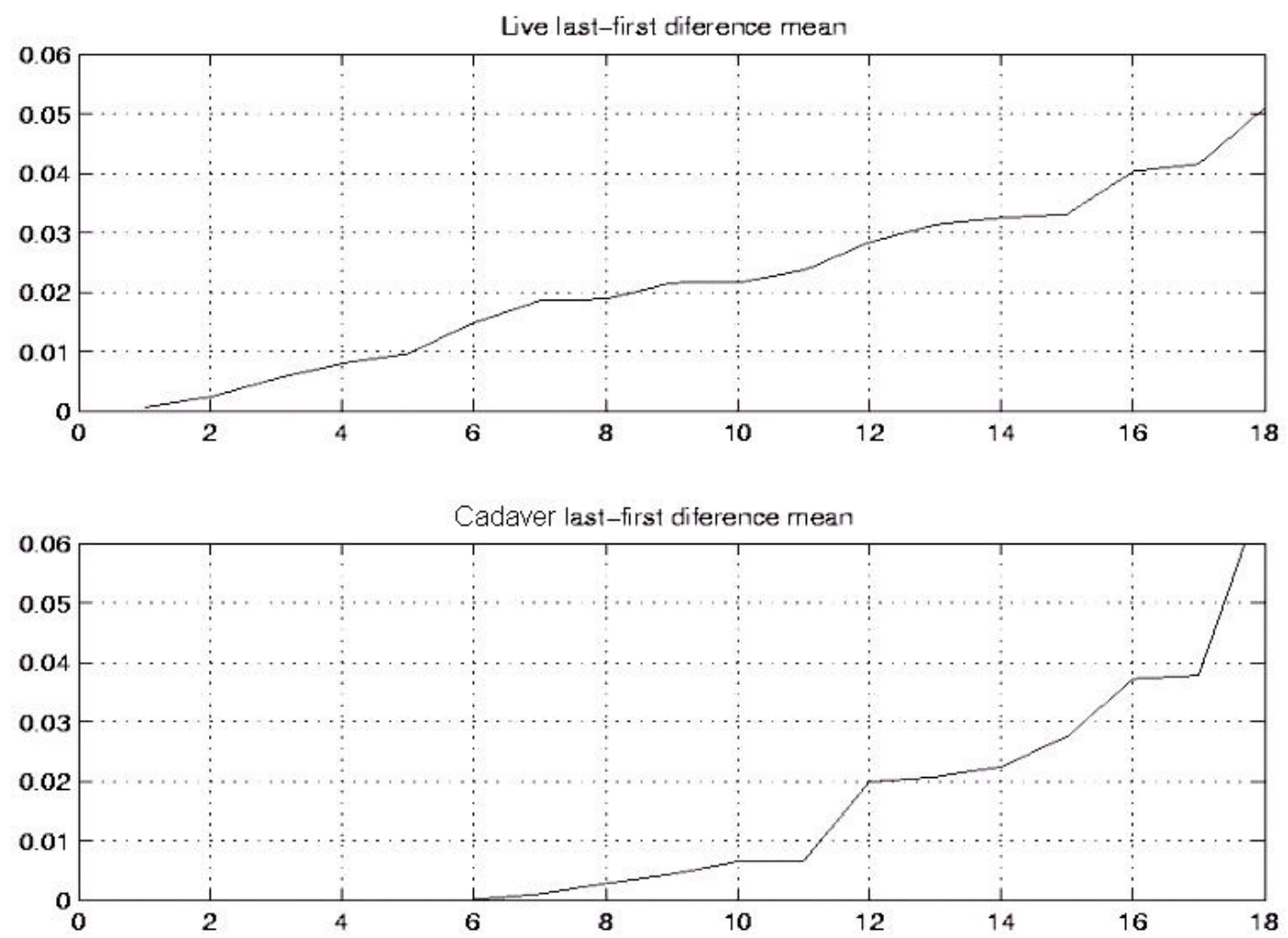

Figure 3-15 Mean of last minus first signals (DM3) for live (top) and cadaver (bottom).

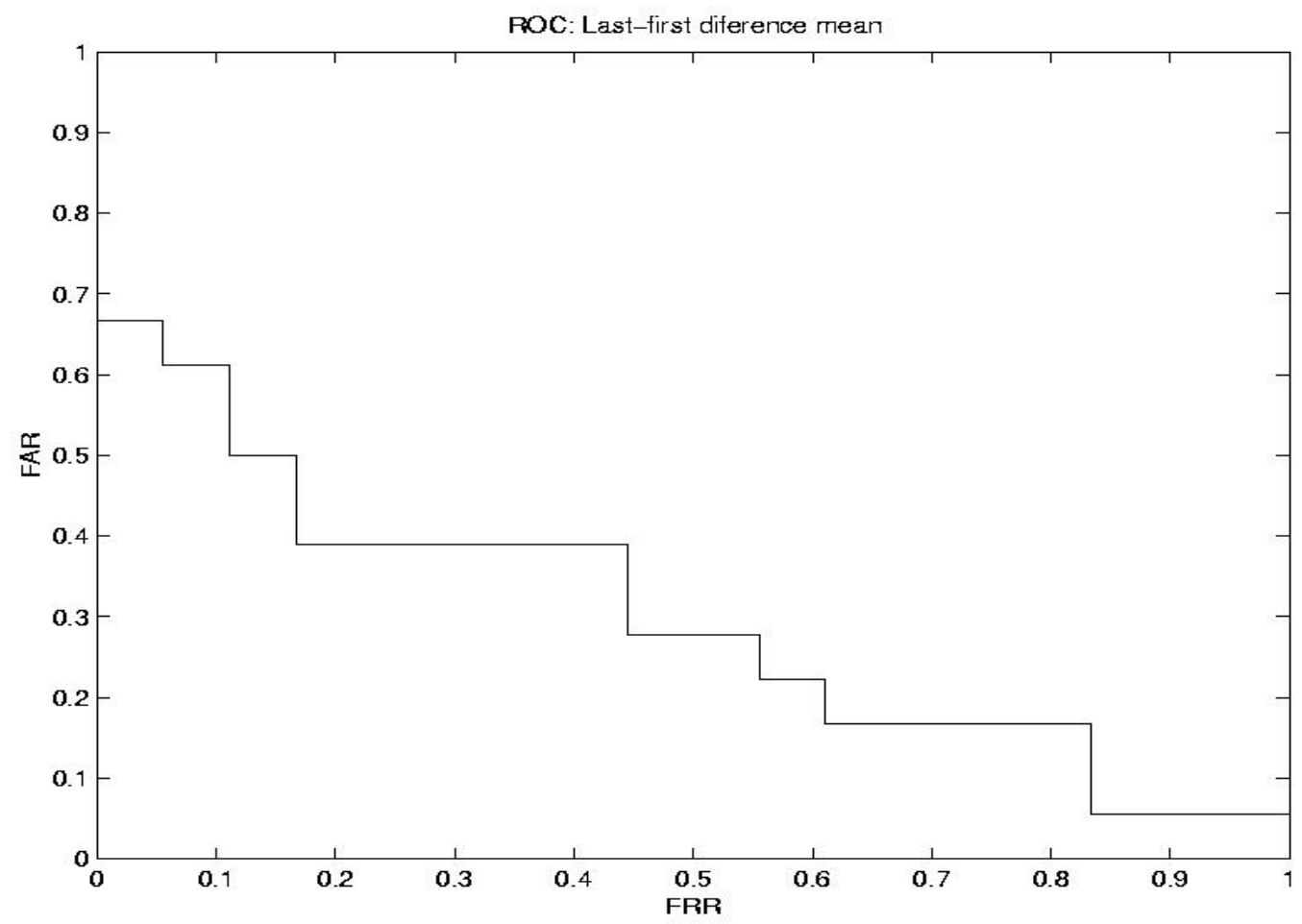

Figure 3-16 The ROC associated with Figure 3-15. 

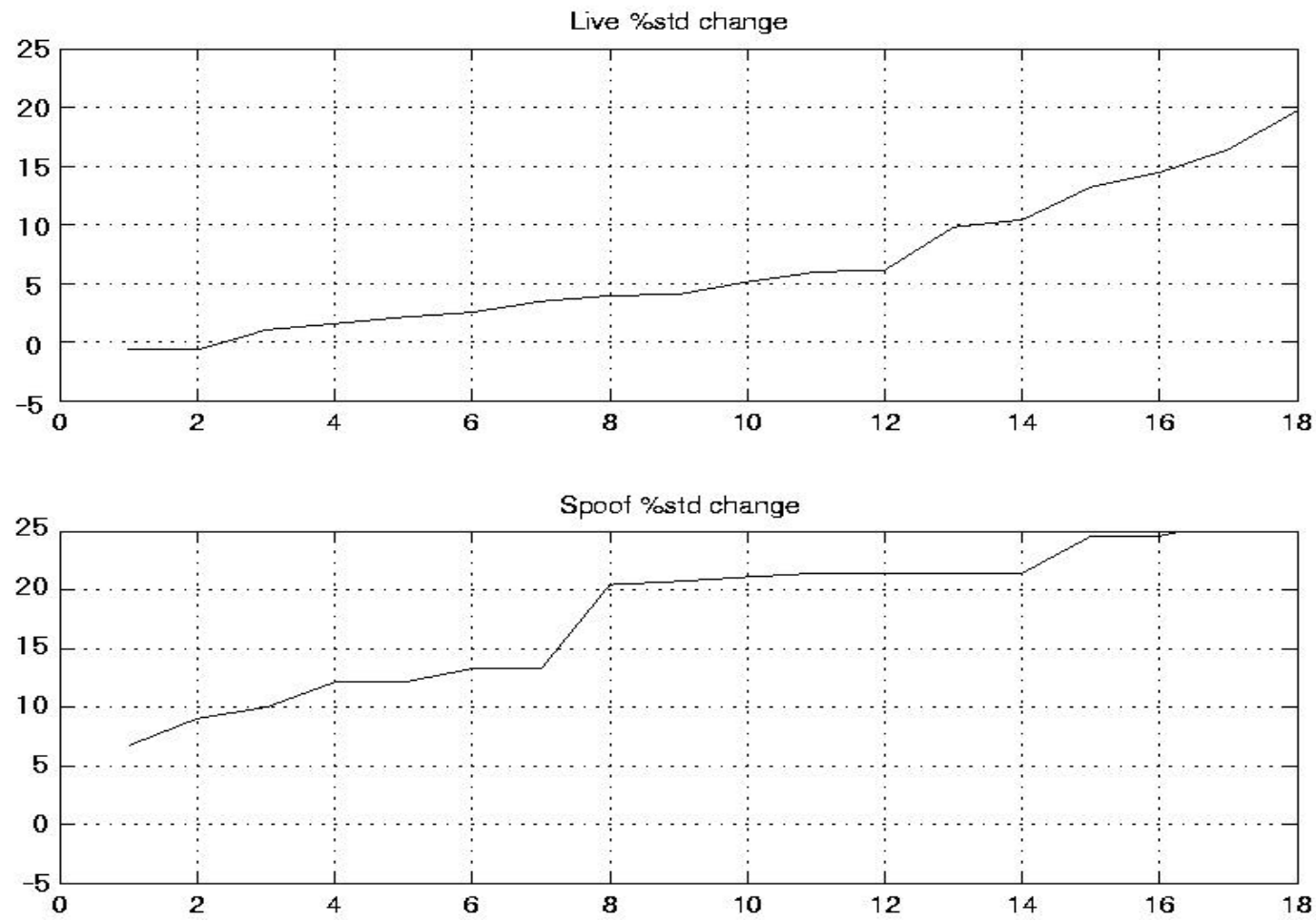

Figure 3-17 Percent change between last-first signal standard deviation (DM4) for live (top) and spoof (bottom).

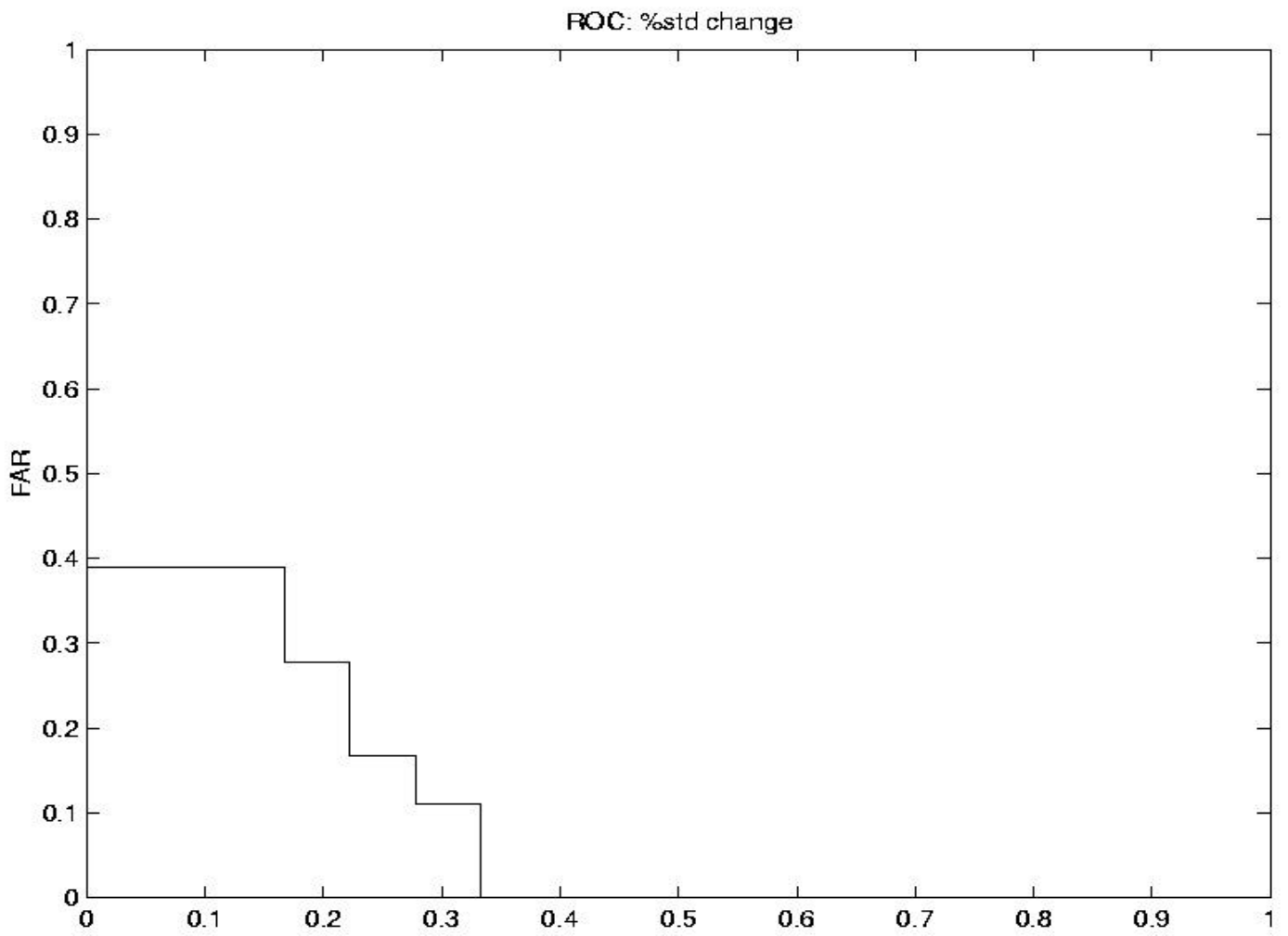

Figure 3-18 The ROC associated with Figure 3-17. 

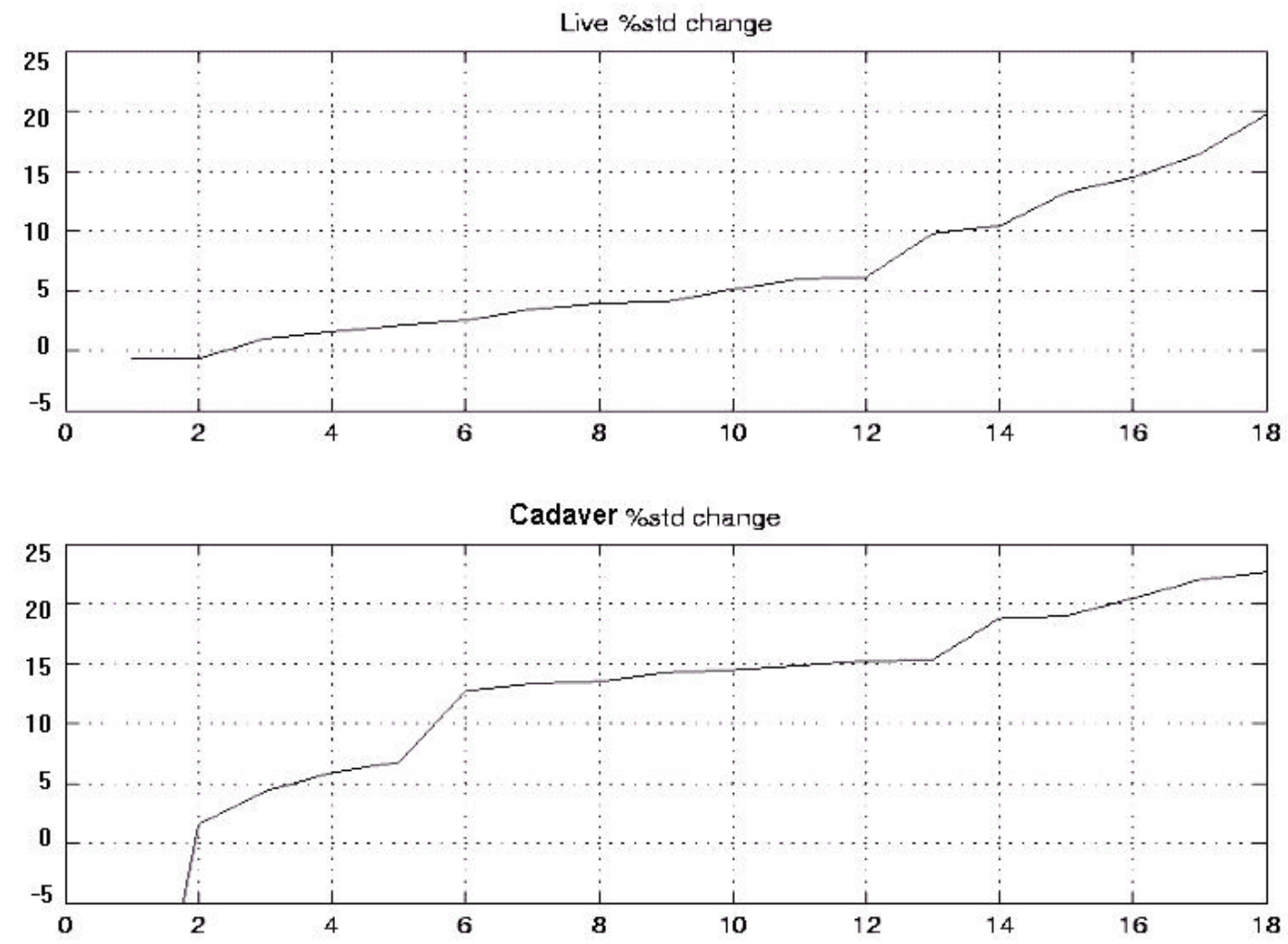

Figure 3-19 Percent change between last-first signal standard deviations (DM4) for live (top) and cadaver (bottom).

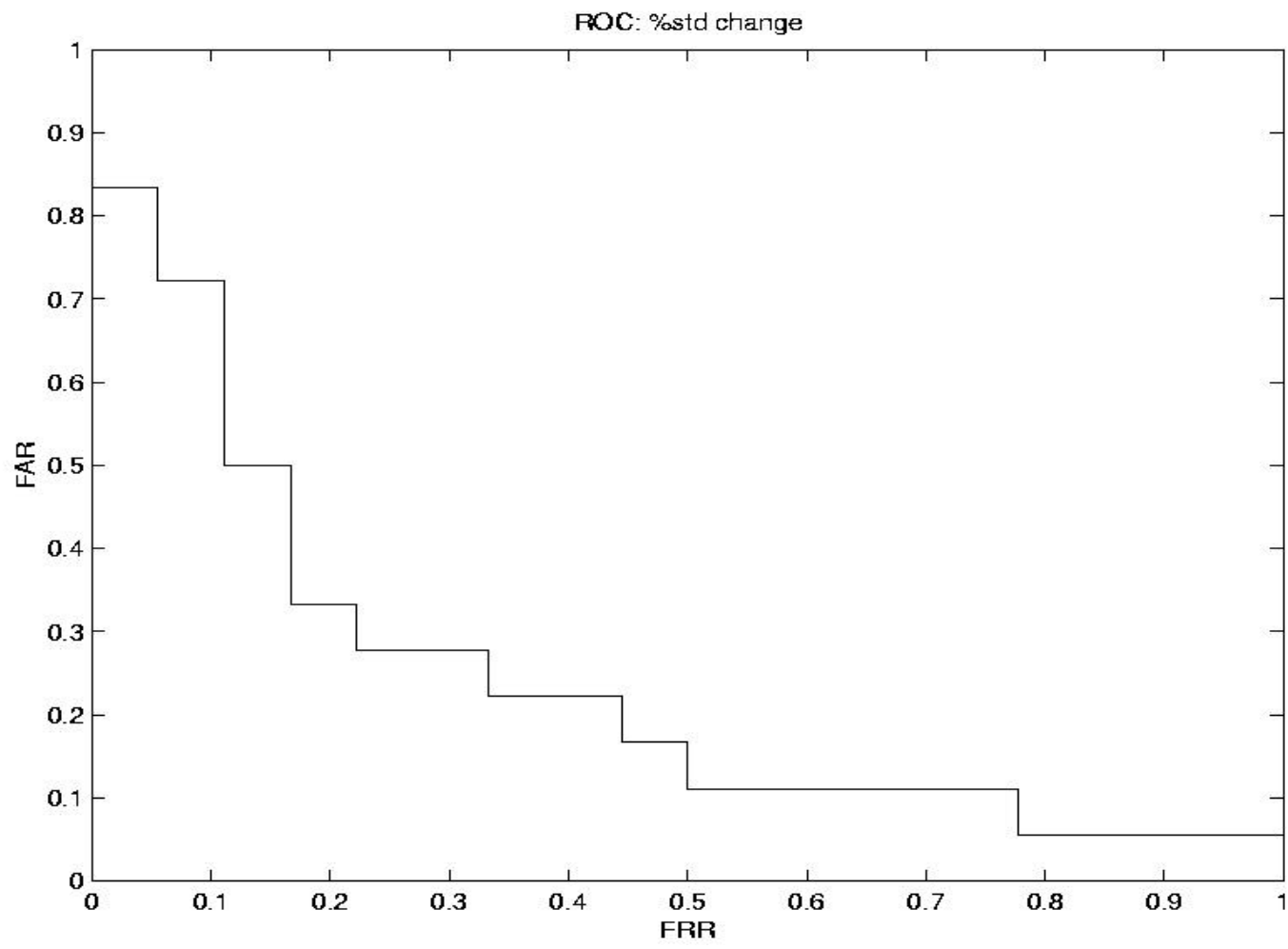

Figure 3-20 The ROC associated with Figure 3-19. 
Chapter 4. Classification 


\section{4-1 Classifier}

As can be seen from the ROCs, None of the measures alone can separate live and cadaver/spoof fingerprints with $100 \%$ sensitivity and specificity (or no false acceptances and no false rejections). However, since the underlying mechanisms for static and dynamic measures are different, one may speculate that some combination of all these measures will provide a better precision than any of the individual measures.

To show the robustness of a combination of static and dynamic measures, a simple decision-maker was first devised using the median values to determine a threshold and simple polling. From the 54 cases under study, two-thirds of data was randomly chosen as training and one-third was reserved for testing. The training set included twelve live fingerprints and the combination of twelve spoof and twelve cadaver fingerprints. For each measure, the medians of the outputs for each set (live and spoof/cadaver) were calculated and the mean of the medians of the two sets for each measure was chosen as a threshold. The thresholds, derived from the training set, were applied to the test sets. The static measure had one case, which was falsely rejected, and no false acceptances. The average of the dynamic measures had no false rejections but two false acceptances. The combination of all the features (polling with equal weights) resulted in no false rejections,

one false acceptance, and two indeterminate states. The result for the test set is as follows ("pass" indicates a "live", and "fail" a "cadaver/spoof" result). 
Table 4-1: Classification of six live fingerprints of the test set, where a "1" indicates live and " 0 " cadaver/spoof. Last row shows polled results from all measures.

\begin{tabular}{|l|c|c|c|c|c|c|}
\hline Case & 1 & 2 & 3 & 4 & 5 & 6 \\
\hline Static & 1 & 1 & 1 & 1 & 0 & 1 \\
\hline Dynamic 1 & 1 & 1 & 1 & 1 & 1 & 1 \\
\hline Dynamic 2 & 1 & 1 & 1 & 1 & 1 & 1 \\
\hline Dynamic 3 & 1 & 1 & 1 & 1 & 1 & 1 \\
\hline Dynamic 4 & 1 & 1 & 1 & 5 & 3 & 5 \\
\hline Sum & 5 & 5 & 5 & Pass & Indeterminate & Pass \\
\hline Result & Pass & Pass & Pass & & & 1 \\
\hline
\end{tabular}

Table 4-2: Classification of six cadaver fingerprints of the test set, where a "1" indicates live and "0" cadaver/spoof. Last row shows polled results from all measures.

\begin{tabular}{|c|c|c|c|c|c|c|}
\hline Case & 1 & 2 & 3 & 4 & 5 & 6 \\
\hline Static & 0 & 0 & 0 & 0 & 0 & 0 \\
\hline Dynamic 1 & 0 & 0 & 0 & 1 & 0 & 0 \\
\hline Dynamic 2 & 0 & 0 & 1 & 1 & 1 & 1 \\
\hline Dynamic 3 & 0 & 0 & 1 & 1 & 1 & 1 \\
\hline Dynamic 4 & 1 & 1 & 0 & 1 & 0 & 0 \\
\hline Sum & 1 & 1 & 2 & 4 & 2 & 2 \\
\hline Result & Fail & Fail & Fail & $\mathrm{X}$ Pass & Fail & Fail \\
\hline
\end{tabular}

Table 4-3: Classification of six spoof fingerprints of the test set, where a "1" indicates live and "0" cadaver/spoof. Last row shows polled results from all measures.

\begin{tabular}{|l|c|c|c|c|c|c|}
\hline Case & 1 & 2 & 3 & 4 & 5 & 6 \\
\hline Static & 0 & 0 & 0 & 0 & 0 & 0 \\
\hline Dynamic 1 & 0 & 1 & 0 & 0 & 0 & 0 \\
\hline Dynamic 2 & 0 & 1 & 1 & 1 & 0 & 0 \\
\hline Dynamic 3 & 0 & 0 & 0 & 0 & 0 & 0 \\
\hline Dynamic 4 & 0 & 1 & 1 & 1 & 0 & 1 \\
\hline Sum & 0 & 3 & 2 & 2 & 0 & 1 \\
\hline Result & Fail & Indeterminate & Fail & Fail & Fail & Fail \\
\hline
\end{tabular}


These results are reasonable but the method is a simple classification. More sophisticated classification may dramatically improve the results. The next section describes the use of a back propagation neural network as a classifier. The results are excellent and the trained neural network was able to classify all the test cases without a single error.

\section{4-2 Neural Networks}

The neural network is a suitable candidate for classification, since each measure is not able to achieve accurate classification on its own. The neural network an approach takes a weighed sum of inputs and calculates a nonlinear combination for output.

The back-propagation neural network (BPNN) is utilized in this work to separate live from cadaver/spoof fingerprints. BPNN uses gradient descent in conjunction with batch input-output training vectors for classification. In fact, using the sigmoid nonlinear transfer function and biases, the BPNN is able to approximate any function with a finite number of discontinuities [20].

As a rule of thumb, the number of hidden layer neurons should be almost one-half of the input layer nodes; so three neurons were used in the hidden layer (Figure 4-1). For convenience of training, bipolar targets $(+1,-1)$ were chosen to denote live and cadaver/spoof, respectively. Log-sigmoid was used for the hidden layer's transfer function and for the output layer's transfer function; both linear and tan-sigmoid were examined. Using different initial random weights during many training sessions, the BPNNs with tan-sigmoid outperformed those of the linear transfer function (output layer), both in terms of training speed and accuracy on the test sets. The five inputs consist of the static measure and four dynamic measures, described in chapter 2. 
For this implementation, two-thirds of the data was used for training and one-third for testing. When presented with the test inputs that it had never seen before, the BPNN classified all of the cases correctly, very significant achievement compared to the previous method.

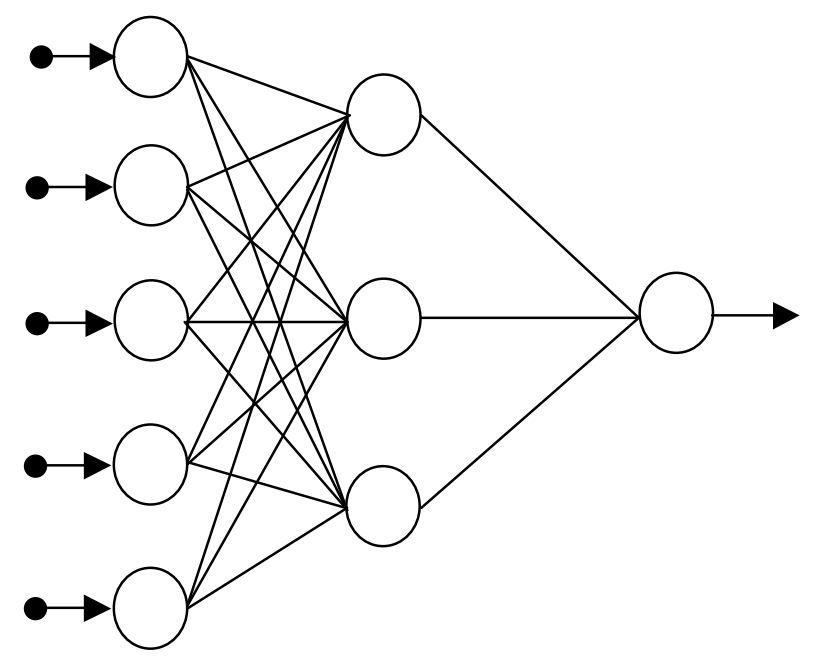

Figure 4-1 Layout of the back-propagation neural network. The inputs are the four dynamic and one static feature. Outputs (close to) +1 or -1 denote live or cadaver/spoof, respectively.

The training and testing of the BPNN depicted above with log-sigmoid and tansigmoid transfer functions was performed using Matlab's neural network toolbox. The network is trained using as many iterations (epochs) as needed until the sum of squared error (SSE) criteria, set at 0.02 in this study, is met (Figure 4-2). The output of the BPNN for the training set is listed in Table 4-4, with the (ideal) set goals of +1 for live, -1 for cadaver/spoof. The output for the test set is listed in Table 4-5. Outputs (close to) +1 or -1 denote live or cadaver/spoof, respectively. 


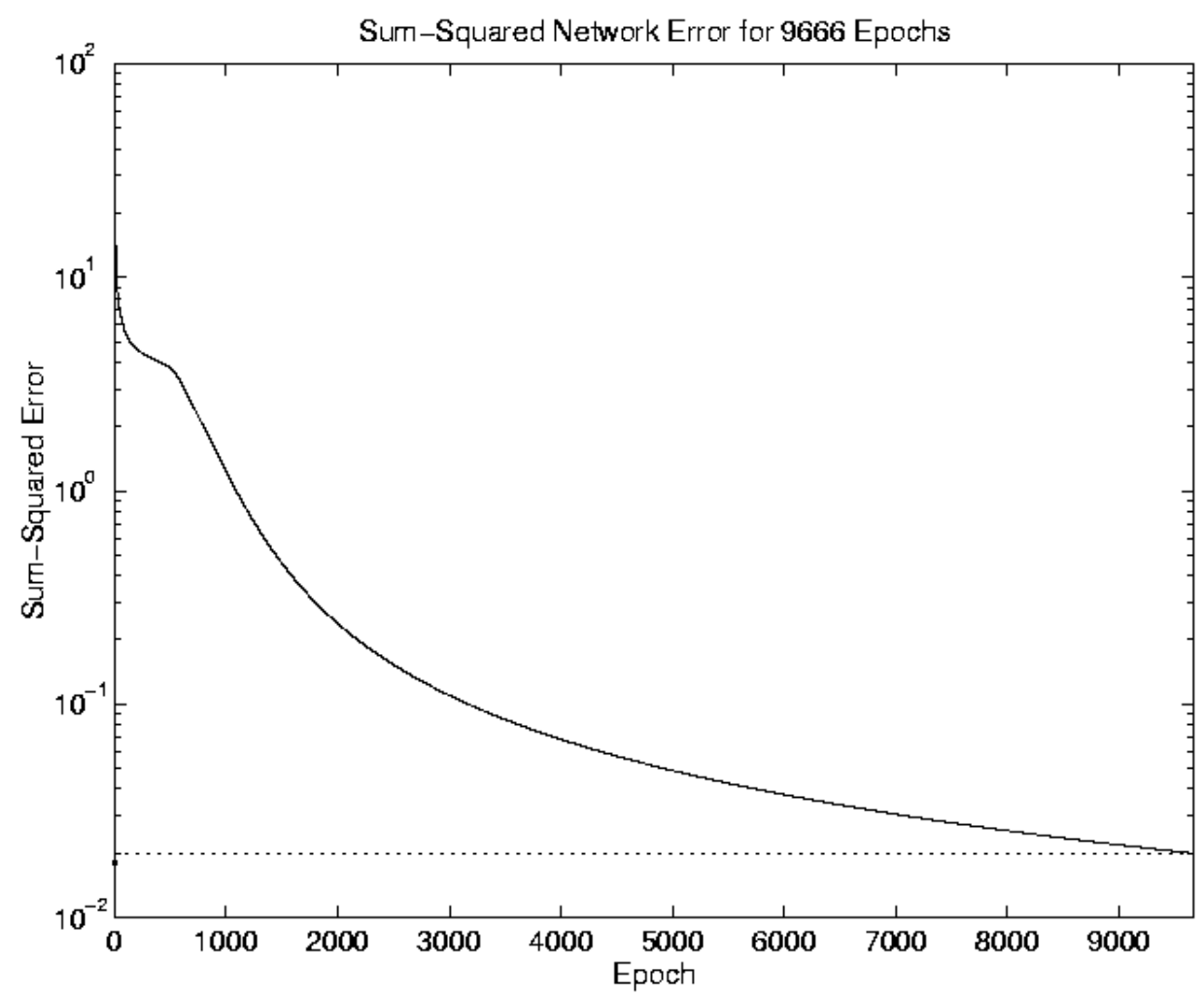

Figure 4-2 Plot of the sum-squared error during training. Error limit is set to 0.02. The number of epochs required for training was 9666

Table 4-4 Output of BPNN for training set

\begin{tabular}{|l|c|c|c|c|c|c|c|c|c|c|c|c|}
\hline Case & $\mathbf{1}$ & $\mathbf{2}$ & $\mathbf{3}$ & $\mathbf{4}$ & $\mathbf{5}$ & $\mathbf{6}$ & $\mathbf{7}$ & $\mathbf{8}$ & $\mathbf{9}$ & $\mathbf{1 0}$ & $\mathbf{1 1}$ & $\mathbf{1 2}$ \\
\hline Live & 0.9677 & 1.0000 & 1.0000 & 0.9085 & 1.0000 & 1.0000 & 1.0000 & 1.0000 & 1.0000 & 1.0000 & 1.0000 & 1.0000 \\
\hline Cadaver & -0.9928 & -0.9922 & -0.9732 & -0.9938 & -0.9885 & -0.9841 & -0.9514 & -0.9923 & -0.9849 & -0.9903 & -0.9940 & -0.9836 \\
\hline Spoof & -0.9439 & -0.9886 & -0.9743 & -0.9912 & -0.9883 & -0.9882 & -0.9876 & -0.9879 & -0.9637 & -0.9925 & -0.9913 & -0.9844 \\
\hline
\end{tabular}

Table 4-5 Output of BPNN for testing set

\begin{tabular}{|l|c|c|c|c|c|c|}
\hline Case & $\mathbf{1}$ & $\mathbf{2}$ & $\mathbf{3}$ & $\mathbf{4}$ & $\mathbf{5}$ & $\mathbf{6}$ \\
\hline Live & 1.0000 & 1.0000 & 1.0000 & 1.0000 & 0.9999 & 1.0000 \\
\hline Cadaver & -0.9950 & -0.9944 & -0.9890 & -0.9938 & -0.9852 & -0.9827 \\
\hline Spoof & -0.9906 & -0.9949 & -0.9939 & -0.8404 & -0.9747 & -0.9852 \\
\hline
\end{tabular}


Table 4-6 Weights and biases of the hidden layer

\begin{tabular}{|c|c|c|c|c|c|c|}
\hline Neuron\Input & Static & $\begin{array}{c}\text { Dynamic } \\
1\end{array}$ & $\begin{array}{c}\text { Dynamic } \\
2\end{array}$ & $\begin{array}{c}\text { Dynamic } \\
3\end{array}$ & $\underset{4}{\text { Dynamic }}$ & Bias \\
\hline 1 & 0.25791334 & -6.4823028 & 1.11036802 & 41.7335775 & 0.10377533 & 1.97862641 \\
\hline 2 & 1.28186592 & 4.59896224 & -0.4272851 & -60.710751 & 3.76033249 & -2.6038195 \\
\hline 3 & 0.32684454 & -1.1206907 & -4.503332 & 58.4514781 & 0.72072412 & 0.5067231 \\
\hline
\end{tabular}

Table 4-7 Weights and biases of the output layer

\begin{tabular}{|c|c|c|c|}
\hline W1 & W2 & W3 & Bias \\
\hline 3.30993063 & 4.72242768 & -5.1894552 & -2.5745411 \\
\hline
\end{tabular}

The results show that no cases in the test or training data were classified incorrectly by the BPNN. Furthermore, the outputs of the test set are very close to the set bipolar targets +1 and -1 , indicating confidence in the classification.

For implementation, the output range of $[-1+1]$ can be divided into three equal subsections as follows: [0.33 1] range for 'live', [-1 -0.33] for 'cadaver/spoof', and (-0.33 0.33 ) for 'indeterminate' states. The indeterminate range can be eliminated if it is undesirable, or in such a case, the system may prompt for repeating the test (please see the last part of the flowchart in Appendix E). 
Chapter 5. Conclusion and Future Work 


\section{5-1 Conclusion}

During this research, a new approach for detection of vitality through fingerprint examination in conjunction with capacitive scanners was introduced. This approach is based on detection of the sweating pattern from two consecutive fingerprints captured during 5 seconds. After mapping two-dimensional fingerprints into one-dimensional signals, two ensembles of measures, namely static and dynamic measures, are extracted from them. Classification is performed using a back propagation neural network trained by the same parameters from example fingerprints. This newly developed algorithm quantifies the sweating pattern and makes a final decision about vitality of the fingerprint.

In conclusion, the method developed in this thesis is a new measure for potential implementation in multi-modal biometrics systems. In addition to its accuracy, it is purely software based, so existing systems can be upgraded without any additional hardware.

\section{5-2 Future Work}

Because the algorithm expands upon the physiological phenomena of perspiration, this approach may have difficulties in cases of perspiration disorders (finger too moist or dry) and other abnormal skin conditions. Nevertheless, one should note that these cases may also have problems when attempting to capture a usable fingerprint (because of abnormal moisture content). This is a subject to further investigation.

Another issue is the orthogonality of the derived features. Specifically, the dynamic features may not independently quantify the event. Future work will be to investigate the overlap and reduce their number or extract a new set of features from the fingerprint signals. Making a fair comparison of different feature sets using the neural net 
classifier is not an easy task, since the neural net does not train consistently even for the same input/target in different training sessions. However, an optimization would definitely lead to a more time efficient algorithm.

Another necessary improvement will be using a larger sample set both for training and for testing the algorithm. The sample set should include wider range of enrollees with different skin conditions in different climates and seasons.

Another possible area of future work would be to decrease the time between the two captures, or to use more than two captures to derive more information. Tradeoffs between precision and speed of vitality verification will need to be addressed. More sophisticated algorithms maybe harder to spoof utilizing features which further quantify sweat diffusion speed and dispersion dynamism.

Finally, this algorithm and its future upgrades should be tested against spoofs which are made to simulate perspiration through artificial pores to evaluate the effort needed to spoof the algorithm.

As with all research, each study produces a new set of questions and potential improvements. In the area of security, complete security (without false rejects) will never be achieved permanently. The goal is to attempt to make spoofing of a system extremely difficult. This work introduces an additional requirement for fingerprint security through a successful method of vitality or "liveness" testing.

\section{5-3 Contributions and Publications}

This work has a patent pending [2] and will be submitted as a paper in January 2000 [21]. 


\section{Bibliography}


[1] Anil Jain, Ruud Bolle, and Sharath Pankanti, , Biometrics, Personal Identification in Networked Society, Chapter 1 "Introduction to Biometrics", edited by Anil Jain, Ruud Bolle, and Sharath Pankanti, Kluwer Academic Publishers, 1999.

[2] Lawrence O'Gorman, Stephanie C. Schuckers, Reza Derakhshani, and Lawrence Hornak; United States Patent, provisional application submitted on October 7, 1999.

[3] Lawrence O'Gorman, Biometrics, Personal Identification in Networked Society, Chapter 2 "Fingerprint Verification", edited by Anil Jain, Ruud Bolle, and Sharath Pankanti, , Kluwer Academic Publishers, 1999.

[4] Kim Stalnaker, Dwayn Bender, Carlos Iga, and Erin Hogbin, "Finger Vitality Tester", senior design project, WVU, 1999, http://www.csee.wvu.edu/ ciga/ .

[5] David W. Osten, Hatim M. Carim, Michael R. Arneson, and Bradford L. Blan; United States Patent, \# 5,719,950 .

[6] "Structure of the Skin", Columbia University College of P \& S Complete Home Medical Guide, http://cpmcnet.columbia.edu/texts/guide/hmg28 0001.html .

[7] M.H.Ross, L.J. Romrell, and E.J. Reith, "The Integumentary System", From Histology: A Text and Atlas, $2^{\text {nd }}$ Ed., Baltimore Williams and Wilkins, 1989, 347-349.

[8] Paul M. Quinton, "Sweating and Its Disorders", Annual Reviews Med., Vol. 34, $1983,429-452$.

[9] Andrea R. Roddy, Jonathan D. Stosz, "Fingerprint Features-Statistical Analysis and System Performance Estimates", Proceedings of the IEEE, Vol. 85, No. 9, September 1997, 1390-1421. 
[10] Yuri A. Chizmadzhev, Andrey V. Indenborn, Peter I. Kuzmin, Sergey V. Galichenko, James C. Weaver, and Russel O. Potts, "Electrical Properties of Skin at Moderate Voltages: Contribution of Appendageal Macropores", Biophysical Journal, Vol. 74, February 1998, 843-856.

[11] Dorin Panescu, Kevin P. Cohen, John G. Webster, and Robert A. Stratbucker, "The Mosaic Electrical Characteristics of the Skin", IEEE Transactions on Biomedical Engineering, Vol. 40, No. 5, May 1995, 434-439.

[12] Dorin Panescu, John G. Webster, and Robert A. Stratbucker, "A Nonlinear Electrical-Thermal Model of the Skin", IEEE Transactions on Biomedical Engineering, Vol. 41, No. 7, July 1994, 672-679.

[13] H.G.L. Coster, K.J. Kim, K. Dahlan, J.R. Smith and C.J.D. Fell, "Characterization of Ultrafiltration Membranes by Impedance Spectroscopy. I. Determination of the Separate Electrical Parameters and Porosity of the Skin and Sublayers", Journal of Membrane Science, Vol. 66, 1992, 19-26.

[14] D. Inglis, L. Manchanda, R. Comizzoli, A. Dickinson, E. Martin, S. Mendis, P. Silverman, G. Weber, B. Ackland, and L. O'Gorman, "SA 17.7: A Robust, 1.8V $250 \mu \mathrm{W}$ Direct-Contact 500 dpi Fingerprint Sensor", 1998 IEEE International Solid-State Circuits Conference, Session 17, Sensor Technology, Paper SA 17.7

[15] Jeong-Woo Lee, Dong-Jin Min, Jiyoun Kim, and Wonchan Kim, "A 600-dpi Capacitive Fingerprint Sensor Chip and Image-Synthesis Technique", IEEE Journal of Solid-State Circuits, Vol. 34, No. 4, April 1999, 469-475

[16] David Halliday, Robert Resnick, Fundamentals of Physics, $3^{\text {rd }}$ Ed., Chapter 27, 1988, John Wiley \& Sons, Inc. 
[17] Martin A. Plonus, Applied Electromagnetics, Chapters 4 \& 5, 1978, McGraw-Hill, Inc.

[18] Teuvo Kohonen, "An Introduction to Neural Computing", Neural Networks, Vol. 1, 1988, Pergamon Press plc., 3-16.

[19] Richard P. Lippmann, "An Introduction to Computing with Neural Nets", IEEE Acoustics, Speech, and Signal Processing Magazine, April 1987, 4-22.

[20] Howard Demuth, Mark Beale," Neural Network Toolbox For Use with MATLAB®", User's Guide Version 3.0, 1997 MathWorks, Inc., Natick, MA.

[21] Reza Derakhshani, Stephanie C. Schuckers, " Determination of Vitality From A Non-Invasive Biomedical Measurement for Use in Fingerprint Scanners ", to be submitted to IEEE Transactions on Pattern Analysis and Machine Intelligence, January 2000.

[22] FPS 100 Solid State Fingerprint Sensor, Preliminary Specification, 02-0003-01 Rev. 2, 3/12/1998, Veridicom Inc., Santa Clara, CA

[23] Geral B. Kasting, and Lisa A. Bowman, "Electrical Analysis of Fresh, Excised Human Skin: A Comparison with Frozen Skin", Pharmaceutical Research, Vol. 7, No. $11,1999,1141-1146$. 
Appendix A: Specifications of FPS100, Solid State Fingerprint Sensor 


\section{Features:}

- 300 X 300 sensor array.

- 500 dpi resolution.

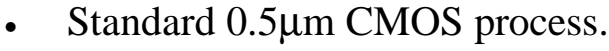

- Sensor pitch: $50 \mu \mathrm{m}$.

- Sensor element: $50 \mu \mathrm{m}$ x $50 \mu \mathrm{m}$, more than $60 \%$ of this area devoted to the sensor plate.

- Array size: $1.5 \mathrm{~cm}$ x $1.5 \mathrm{~cm}$.

- Sensor integration time: $\sim 1 \mu$ s.

- Row read-out: $\quad 50 \mu$ s.

- Maximum frames per second: 60.

- Stand-by power dissipation at 1.8V, 10 Frames/sec: $110 \mu \mathrm{W}$.

- Active power at 60 Frames/sec: $250 \mu \mathrm{W}$.

- False Accept Ratio (using commercial fingerprint recognition software): $<1 \%$.

- 8-bit microprocessor interface.

- VSPA 80/1 (similar to $24 \mathrm{~mm}$ x $24 \mathrm{~mm}$ TQFP) or 169-pin, 27 x 27 mm BGA.

\section{Absolute Maximum Ratings:}

- Storage Temperature: $-65^{\circ}$ to $+150^{\circ}$.

- DC Voltage Applied to any pins: -5.0 to $+7.0 \mathrm{~V}$.

- Electrostatic Discharge voltage: >2000 Volts.

- Latch up Current: >100 mA.

\section{Operating Range:}

- Ambient Temperature: $-0^{\circ}$ to $+70^{\circ}$.

- $\mathrm{V}_{\mathrm{DD}}$ (Digital Supply voltage): -4.3 to $+5.5 \mathrm{~V}$.

- $\mathrm{V}_{\mathrm{DDA}}$ (Analog Supply voltage): -3.0 to $+5.5 \mathrm{~V}$.

- Oscillator Frequency: $10 \mathrm{MHz}$ to $40 \mathrm{MHz}$.

[22], [14] 


\section{Appendix B: Finger Frequency Response Tests}




\section{Test Set-up for Finger Frequency Response Measurement}

HP 33120A

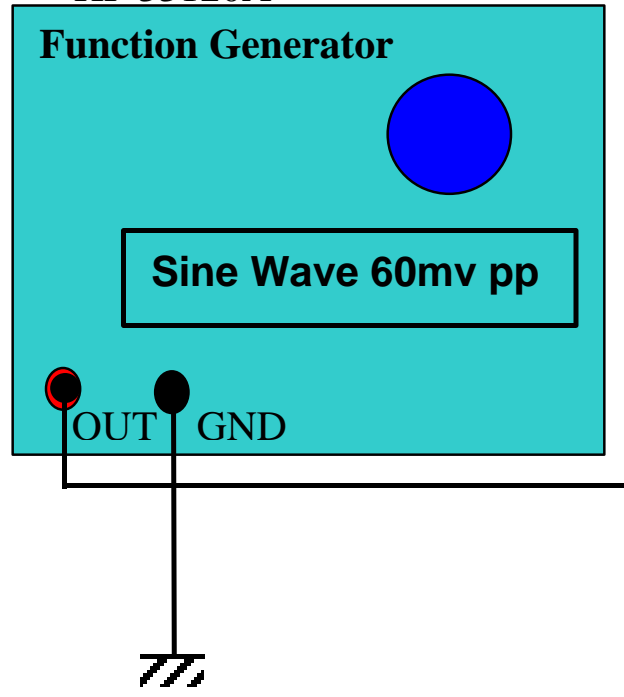

HP54602B



Electrodes: HP M2253A, Solid Gel Disposable Diagnostic Electrode

Frequencies:100Hz, 1kHz, 10kHz, 100kHz, 1MHz, 10Mhz (60mv pp Sine Wave) 


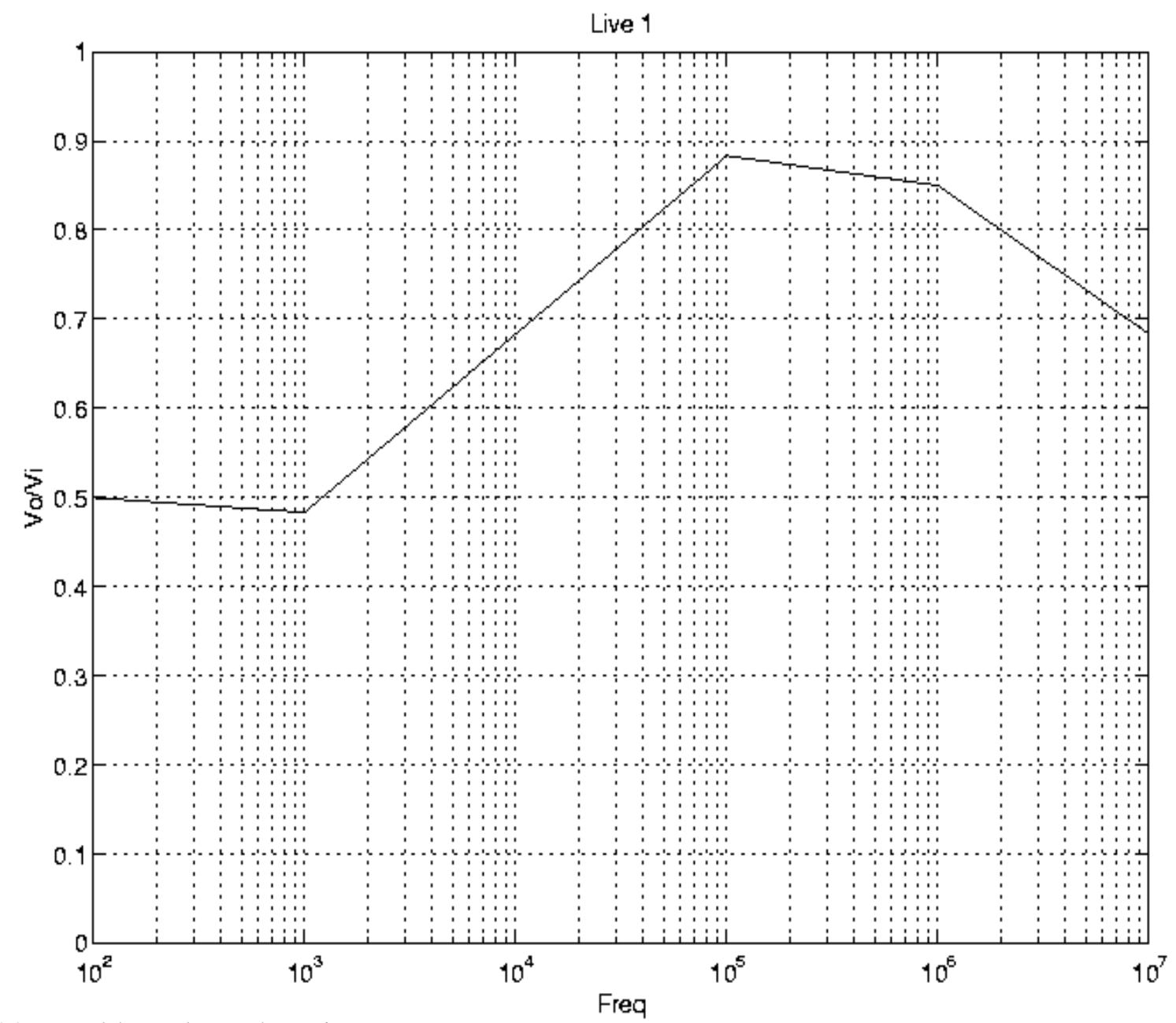

28 yrs old, Male, Index Finger 


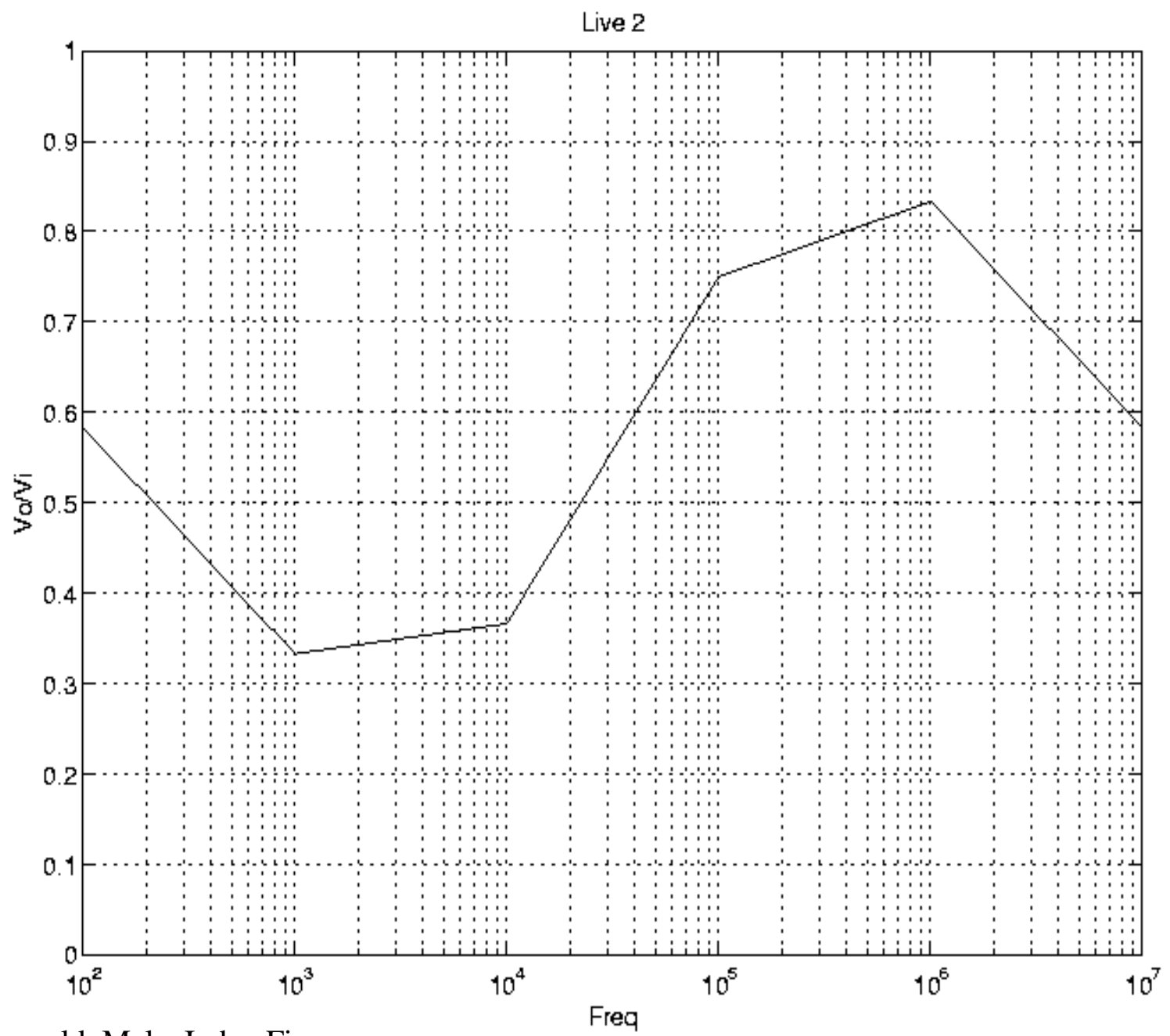

26 yrs old, Male, Index Finger 


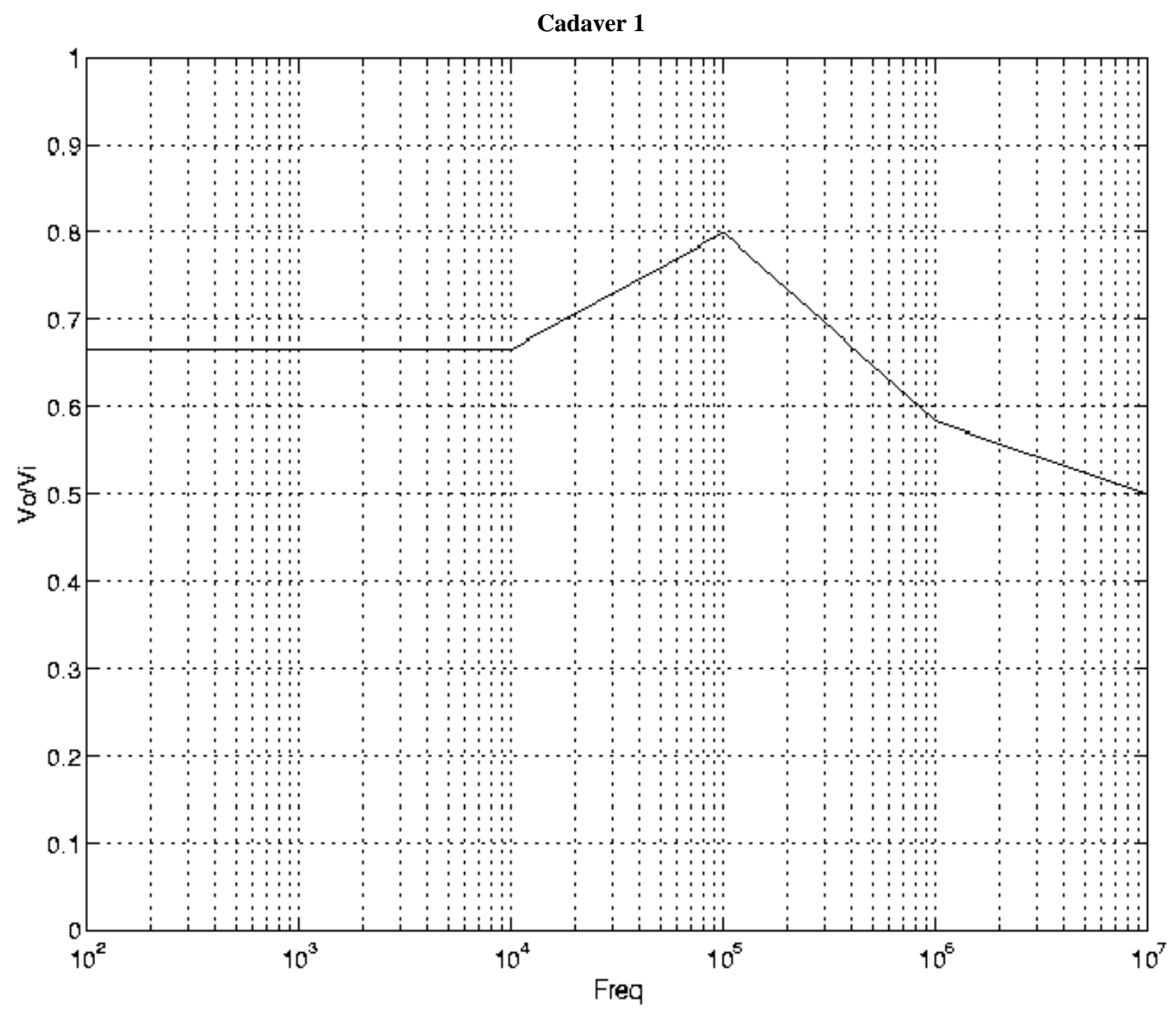

81 yrs old, Male, Index Finger, Year of Death: 1997 


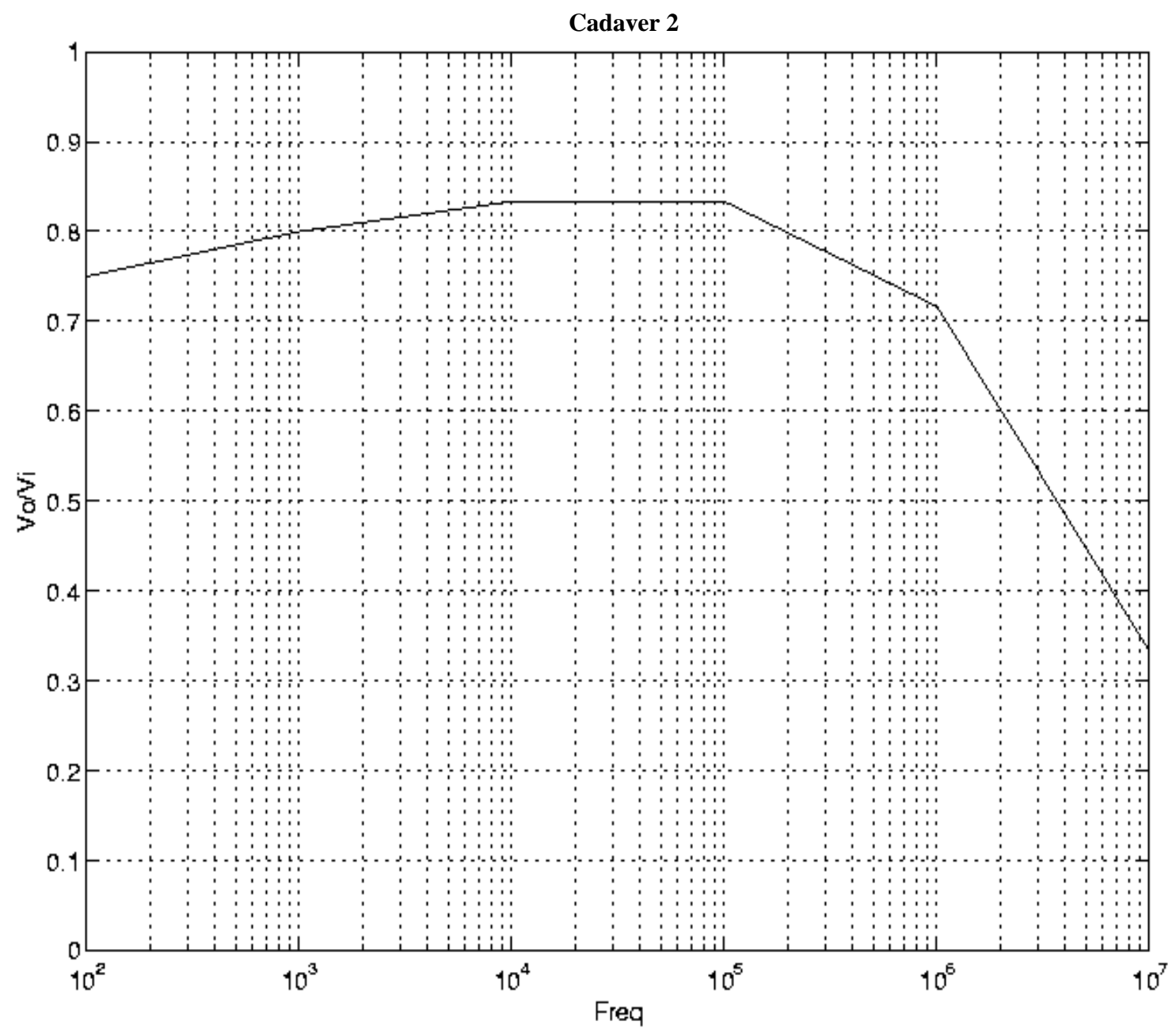

48 yrs old, Male, Index Finger, Year of Death: 1989 
Appendix C: A Comparison Between Fresh and Frozen Skin 
In our experiments with cadavers, all but two of the cadaver fingers had been frozen for storage. A question arises here: How different is the electrical properties of human skin, after being frozen and thawed? The literature indicates that frozen skin is similar to fresh skin in most aspects [23]. First, frozen skin is more permeable to sodium ions during passive diffusion, is less permselective for sodium versus chloride in constant-current iontophoresis, and has lower DC resistance. The current-voltage characteristics look similar, but frozen skin i-v curves are more symmetric. As an overall conclusion, frozen skin, is not very different from fresh skin with respect to this study. Therefore, the use of thawed frozen skin, instead of fresh skin, is justified [23]. 


\section{Appendix D: Sample Fingerprints and Related Outputs}


Case 1 Live

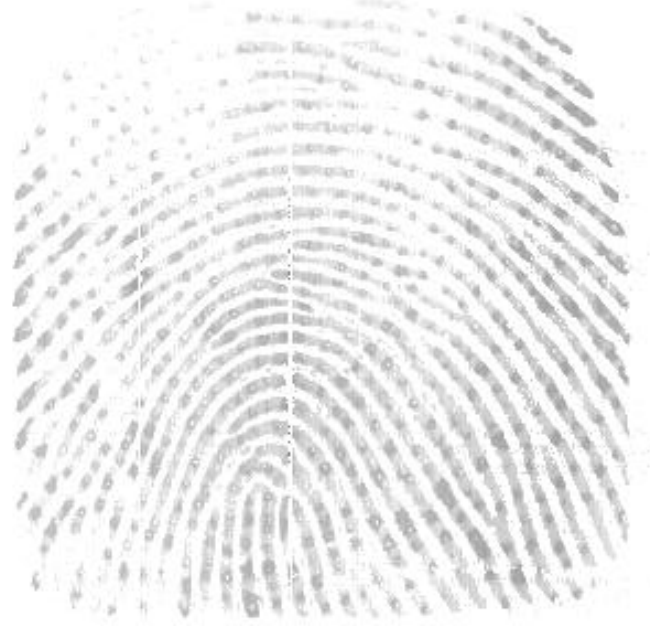

First Capture

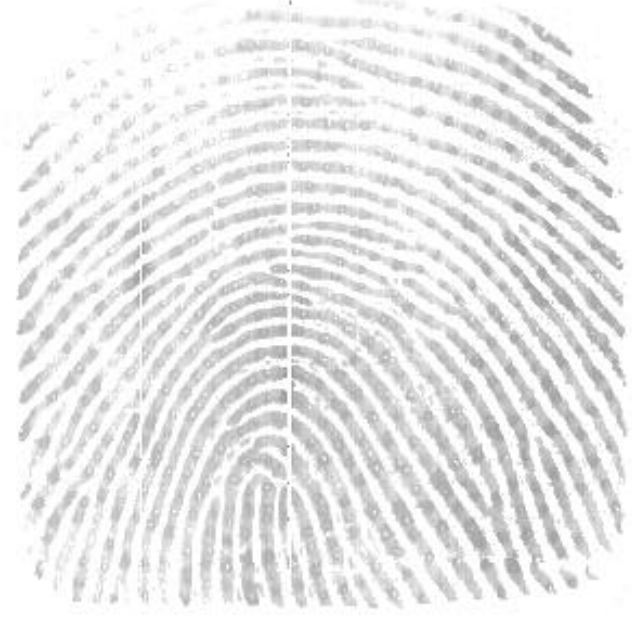

Last Capture

FFT of mean of first individual contours

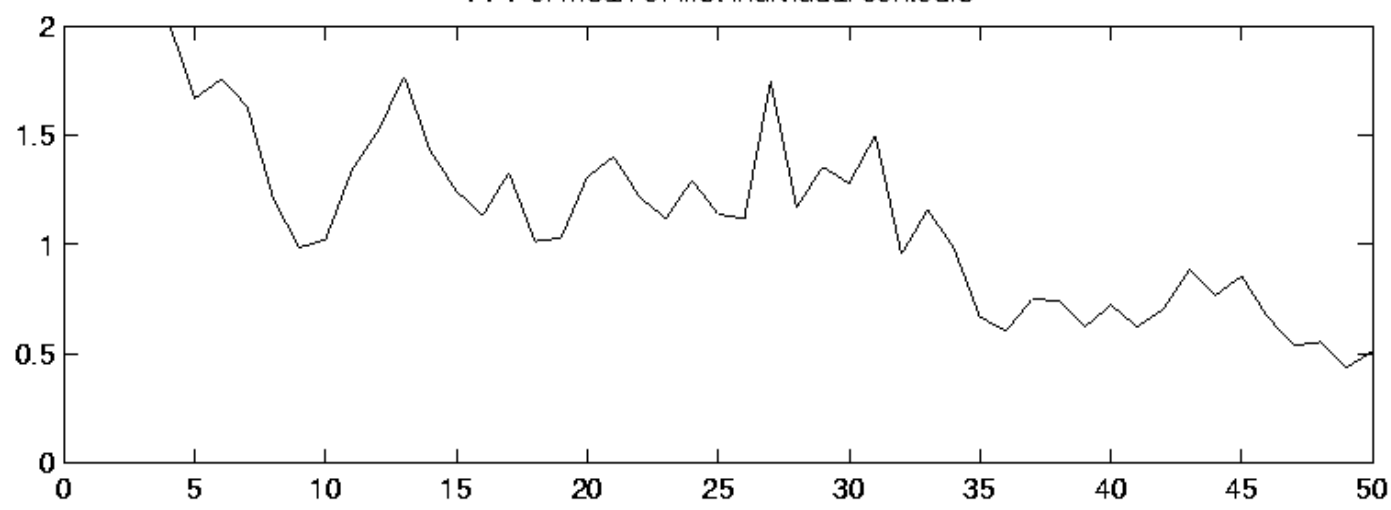

FFT of mean of last individual contours

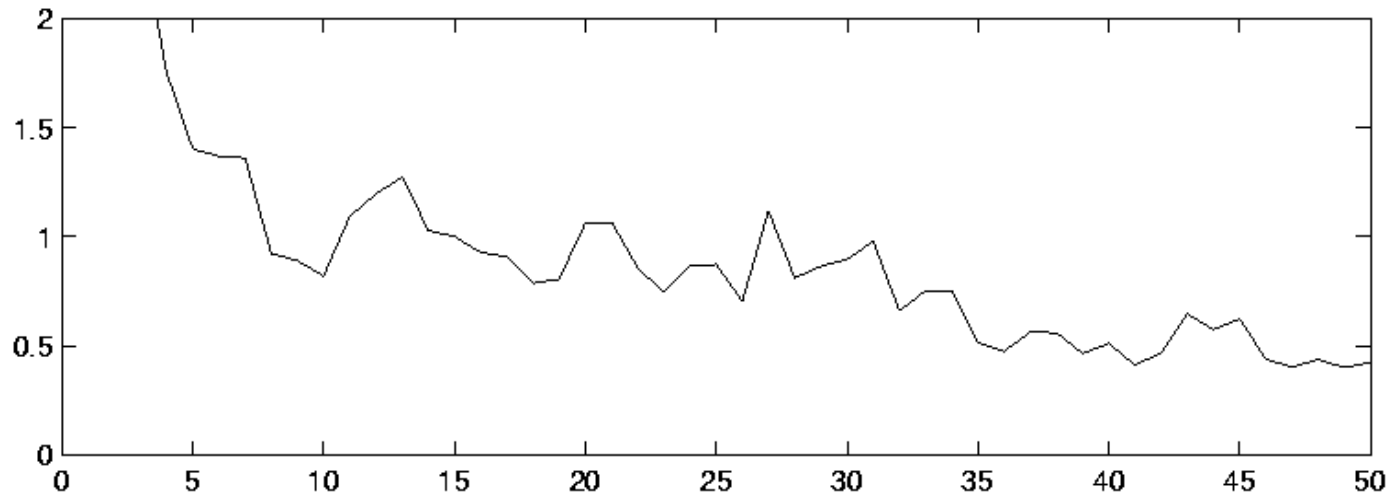

FFT for Static Measure 


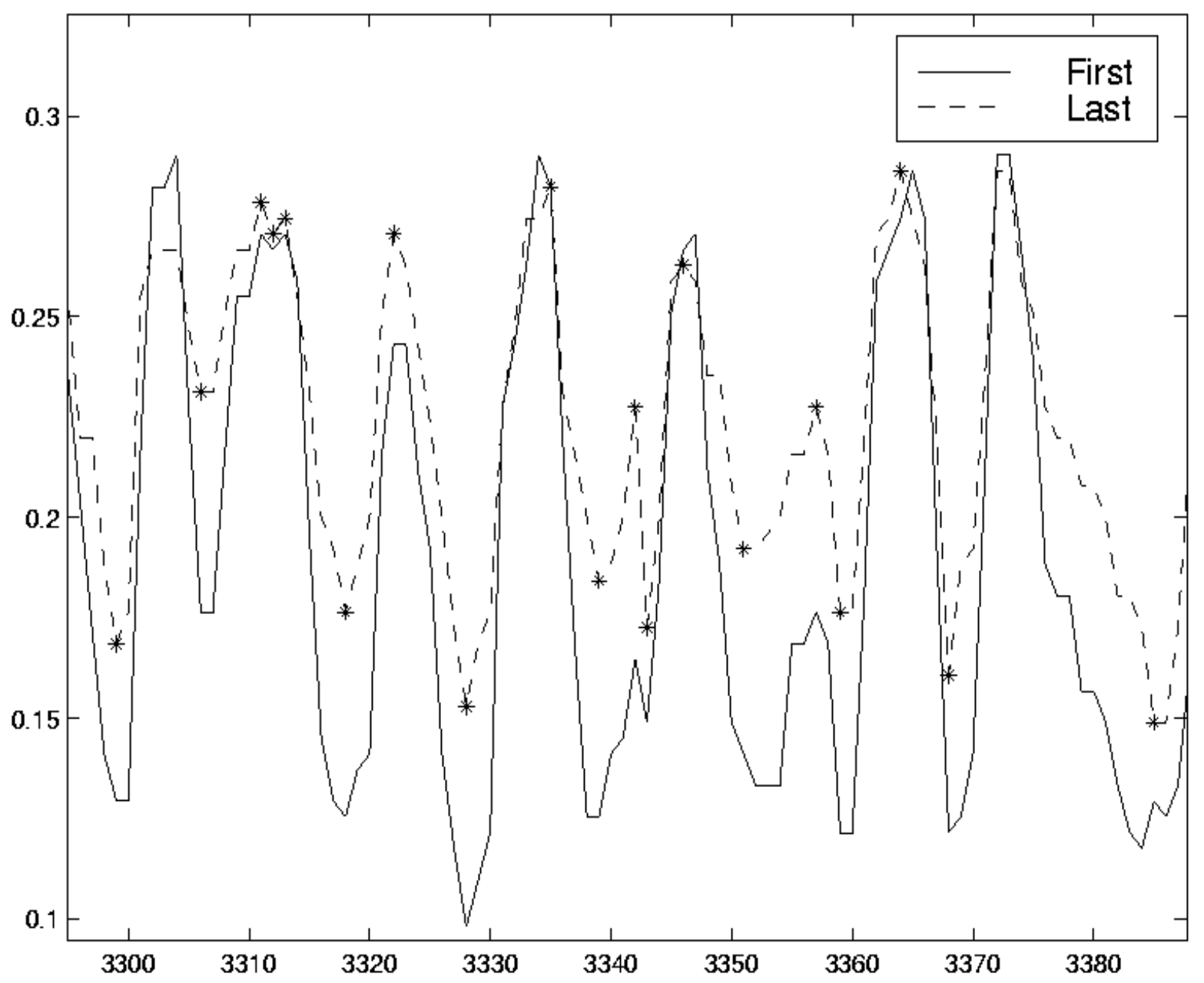

First versus last capture fingerprint signals for dynamic measures. Extremums denoted by 


\section{Extracted Parameters:}

First Mean $=0.1498$ Last Mean $=0.1901$

MeanPercentChange $=26.896$

FirstFFTstd $=5.0664 \quad$ LastFFTstd $=4.2075$

FirstFFTstdLPF $=5.3067$ LastFFTstdLPF $=4.3630$

First std $=0.0851$ Last std $=0.0694$ stdPercentChange $=-18.399$

Last $-F i r s t D i f f M e a n=0.040$ Last $-F i r s t D i f f S t d=0.032$

Original: SumOfFirstMINS=99.2314 SumOfFirstMAXS=138.5804

SumOfLastMINS $=140.2000 \quad$ SumOfLastMAXS $=163.2549$

Original NORMALIZED:SumOfFirstMINS=0.132840

SumOfFirstMAXS $=0.153128$

SumOfLastMINS $=0.187684$ SumOfLastMAXS $=0.180392$

Filtered: SumOfFirstMINS=83.1144 SumOfFirstMAXS=155.8240

SumOfLastMINS=121.3925 SumOfLastMAXS=179.7685

Filtered NORMALIZED:SumOfFirstMINS $=0.103763$

SomeOfFirstMAXS $=0.194537$

SumOfLastMINS=0.151551 SumOfLastMAXS=0 .224430

Original: FirstToLastMAXSgrowth=24.6745

FirstToLastMINSgrowth $=40.9686$

FirstMINStoMAXS sum=39.3490 LastMINStoMAXSsum=23.0549

Original NORMALIZED: FirstToLastMAXSgrowth=0.027265

FirstToLastMINSgrowth $=0.054844$

FirstMINStoMAXS sum $=0.043480$ LastMINStoMAXSsum $=0.025475$

Filtered: FirstToLastMAXSgrowth $=23.9445$

FirstToLastMINSgrowth $=38.2781$

FirstMINStoMAXSsum $=72.7096$ LastMINStoMAXSsum $=58.3760$

Filtered NORMALIZED: FirstToLastMAXSgrowth=0.030805

First ToLastMINSgrowth $=0.051147$

FirstMINStoMAXS sum $=0.049125$ LastMINStoMAXSsum $=0.028783$

Original NORMALIZED: FirstTotalSwing=0.029716

Last Totalswing $=0.022680$

Filtered NORMALIZED: FilteredFirstTotalSwing $=0.027028$

FilteredLastTotalSwing $=0.020065$

stdFFTMeanFirst $=0.5659$ FFTstdMeanLast $=0.4719$ 


\section{Case 2 Live}

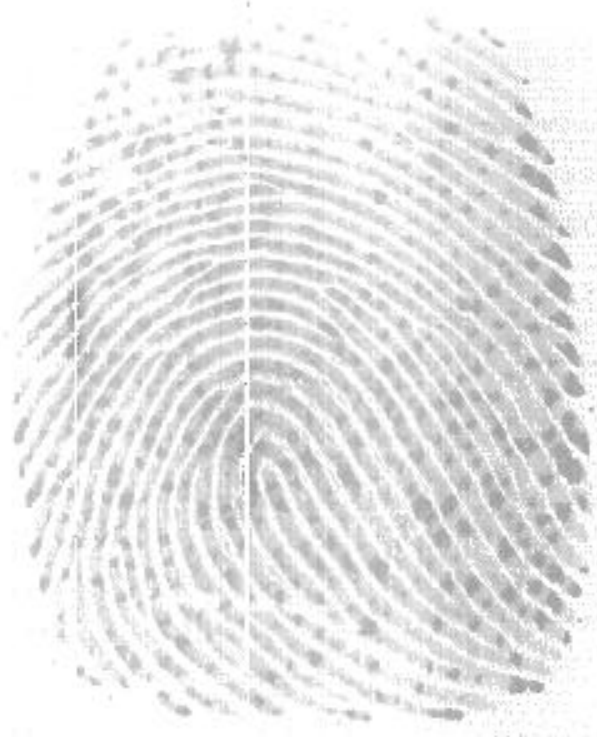

First Capture

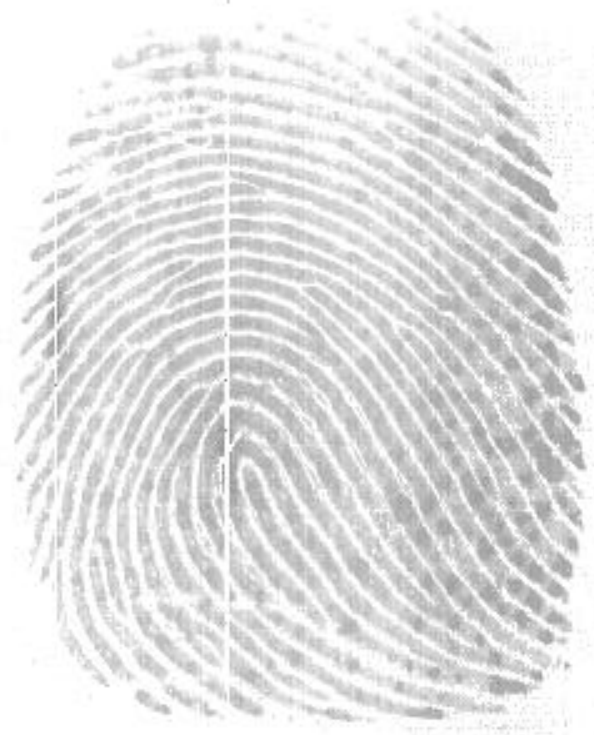

Last Capture

FFT of mean of first individual contours

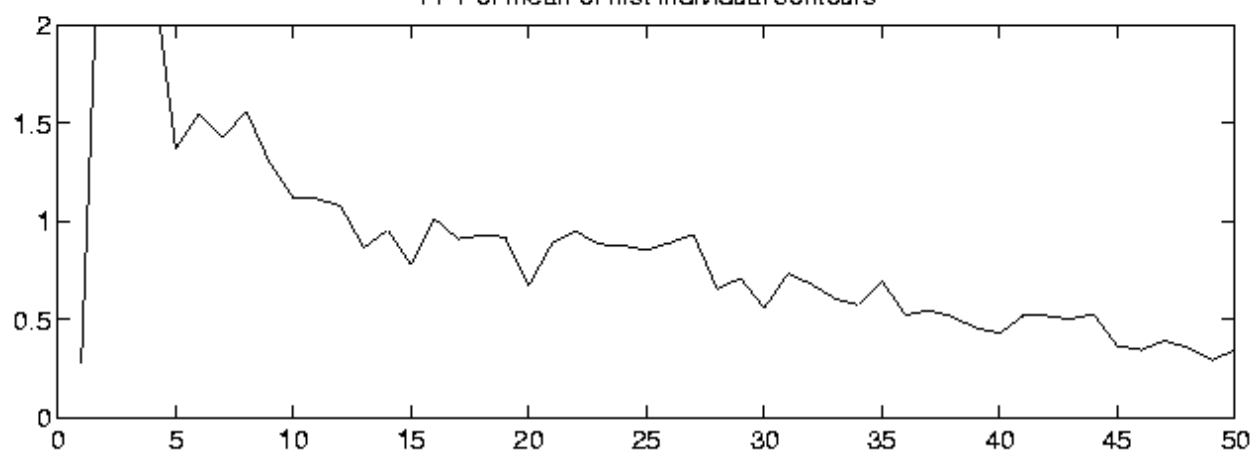

FFT of mean of last individual contours



FFT for Static Measure 


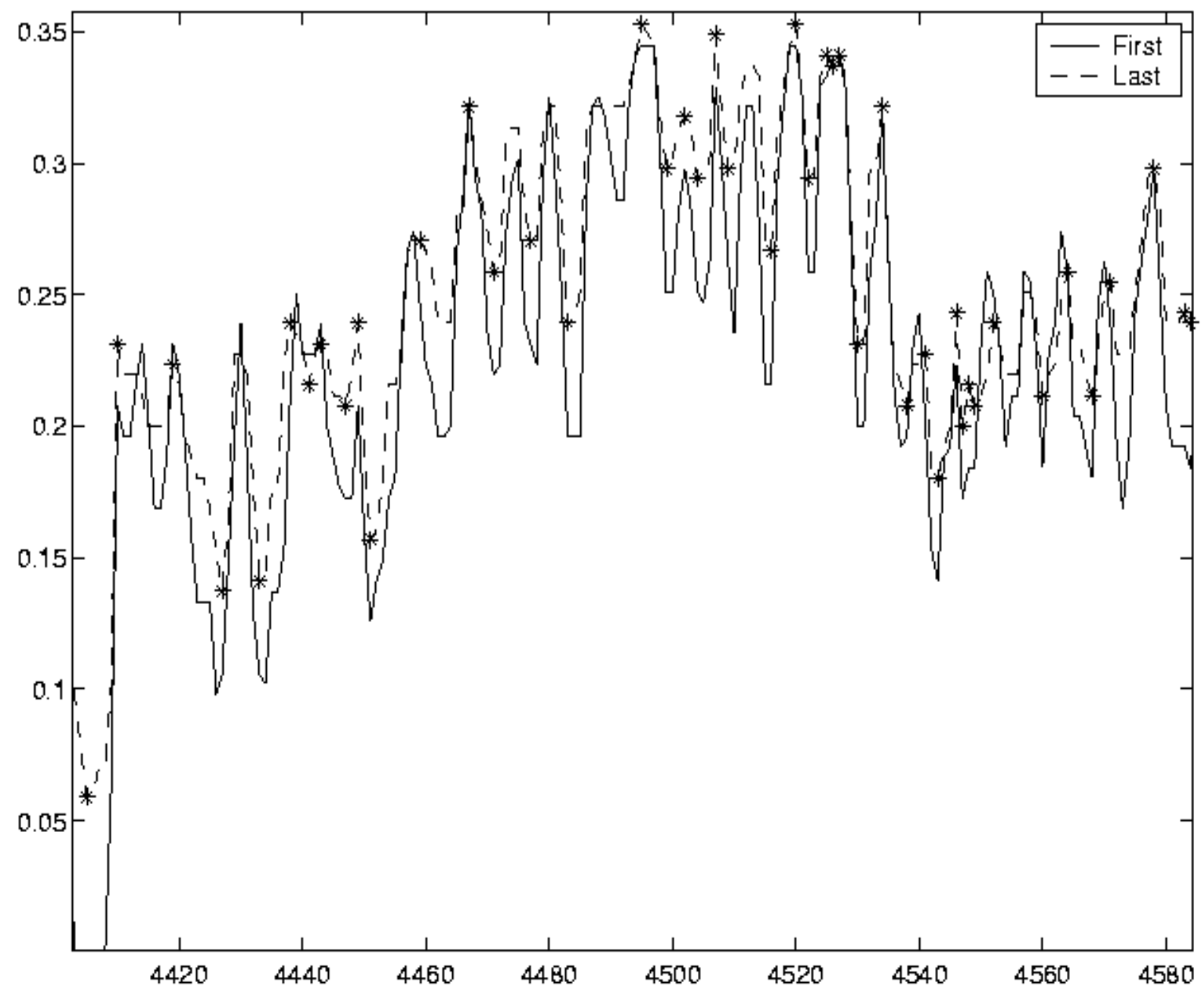

First versus last capture fingerprint signals for dynamic measures. Extremums denoted by 


\title{
Extracted Parameters:
}

\author{
First Mean $=0.1674$ Last Mean $=0.2005$ \\ MeanPercentChange $=19.820$ \\ FirstFFTstd $=4.3377 \quad$ LastFFTstd $=3.7021$ \\ FirstFFTstdLPF $=4.5441$ LastFFTstdLPF $=3.8925$ \\ First std $=0.0784$ Last std $=0.0655$ stdPercentChange $=-16.506$ \\ Last - FirstDiffMean $=0.033$ Last - FirstDiffStd=0.041 \\ Original: SumOfFirstMINS $=122.7843$ SumOfFirstMAXS $=155.1098$ \\ SumOfLastMINS=154.1961 SumOfLastMAXS=176.2745 \\ Original NORMALIZED:SumOfFirstMINS=0.160084 \\ SumOfFirstMAXS $=0.171014$ \\ SumOfLastMINS=0.201038 SumOfLastMAXS $=0.194349$ \\ Filtered: SumOfFirstMINS=103.3961 SumOfFirstMAXS=155.1171 \\ SumOfLastMINS $=132.0024$ SumOfLastMAXS $=175.5296$ \\ Filtered NORMALIZED:SumOfFirstMINS $=0.132900$ \\ SomeOfFirstMAXS $=0.199123$ \\ SumOfLastMINS=0.169669 SumOfLastMAXS=0.225327 \\ Original: FirstToLastMAXSgrowth=21.1647 \\ FirstToLastMINSgrowth $=31.4118$ \\ FirstMINStoMAXS sum=32.3255 LastMINStoMAXSsum=22.0784 \\ Original NORMALIZED: FirstToLastMAXSgrowth $=0.023335$ \\ FirstToLastMINSgrowth $=0.040954$ \\ FirstMINStoMAXS sum $=0.035640$ LastMINStoMAXSsum $=0.024342$ \\ Filtered: FirstToLastMAXSgrowth=20.4125 \\ FirstToLastMINSgrowth $=28.6062$ \\ FirstMINStoMAXSsum $=51.7209$ LastMINStoMAXSsum $=43.5272$ \\ Filtered NORMALIZED: FirstToLastMAXSgrowth $=0.027169$ \\ First ToLastMINSgrowth $=0.040375$ \\ FirstMINStoMAXS sum $=0.041496$ LastMINStoMAXSsum $=0.028342$ \\ Original NORMALIZED: FirstTotalSwing=0.028114 \\ LastTotalswing $=0.021751$ \\ Filtered NORMALIZED: FilteredFirstTotalSwing=0.022533 \\ FilteredLast TotalSwing $=0.016841$ \\ stdFFTMeanFirst $=0.4691 \quad$ FFTstdMeanLast $=0.3777$
}




\section{Case 3 Live}

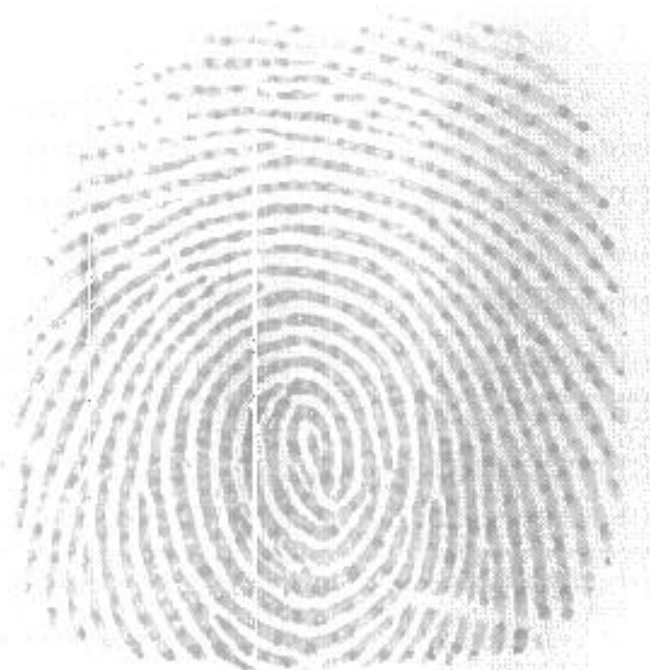

First Capture

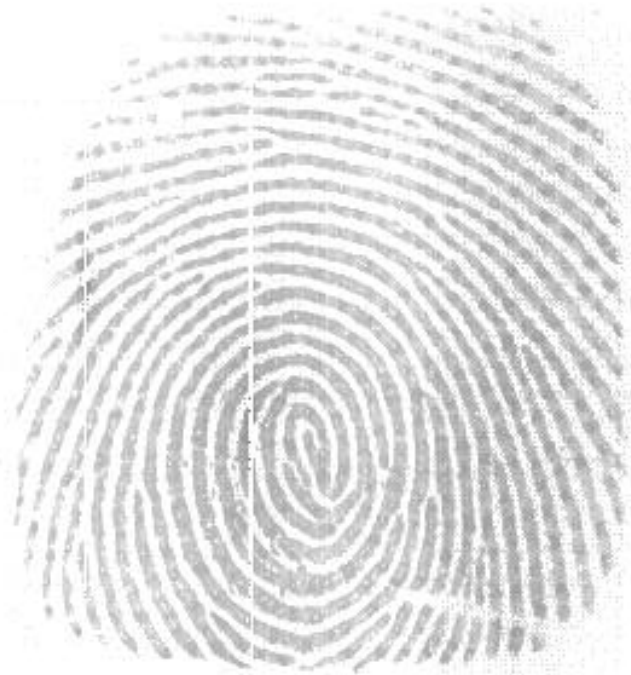

Last Capture

FFT of mean of first individual contours

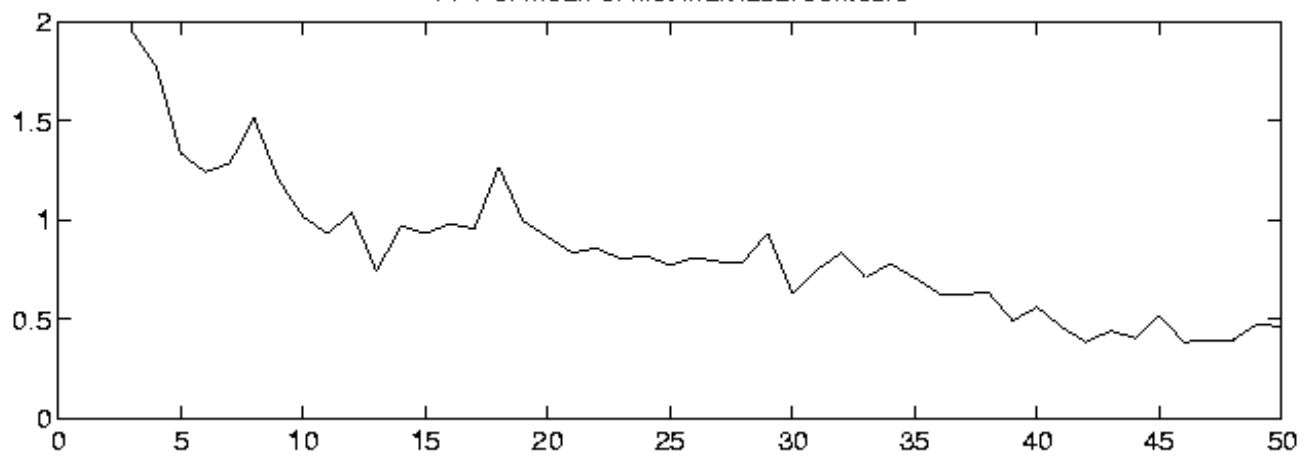

FFT of mean of last individual contours

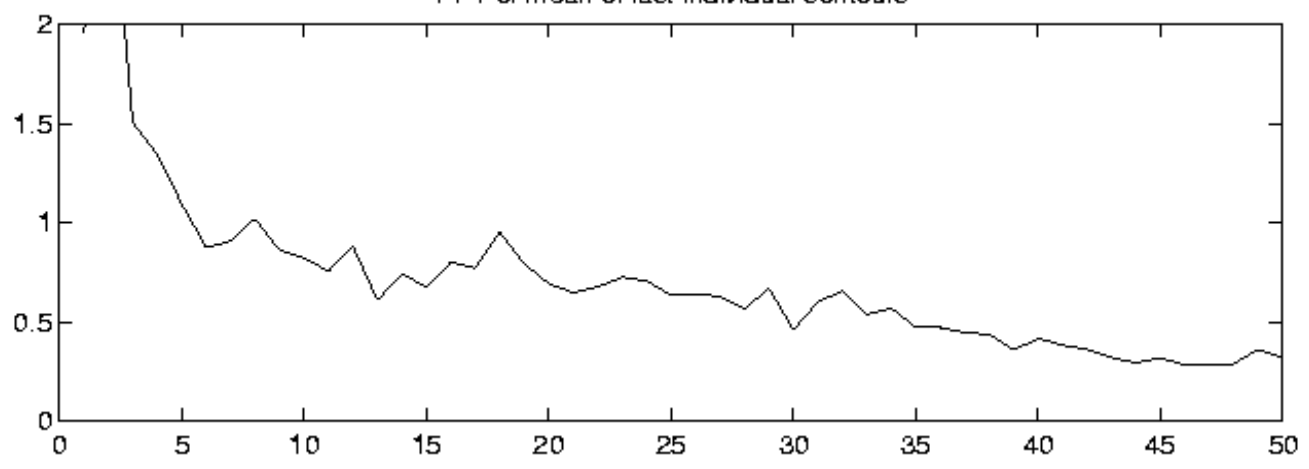

FFT for Static Measure 


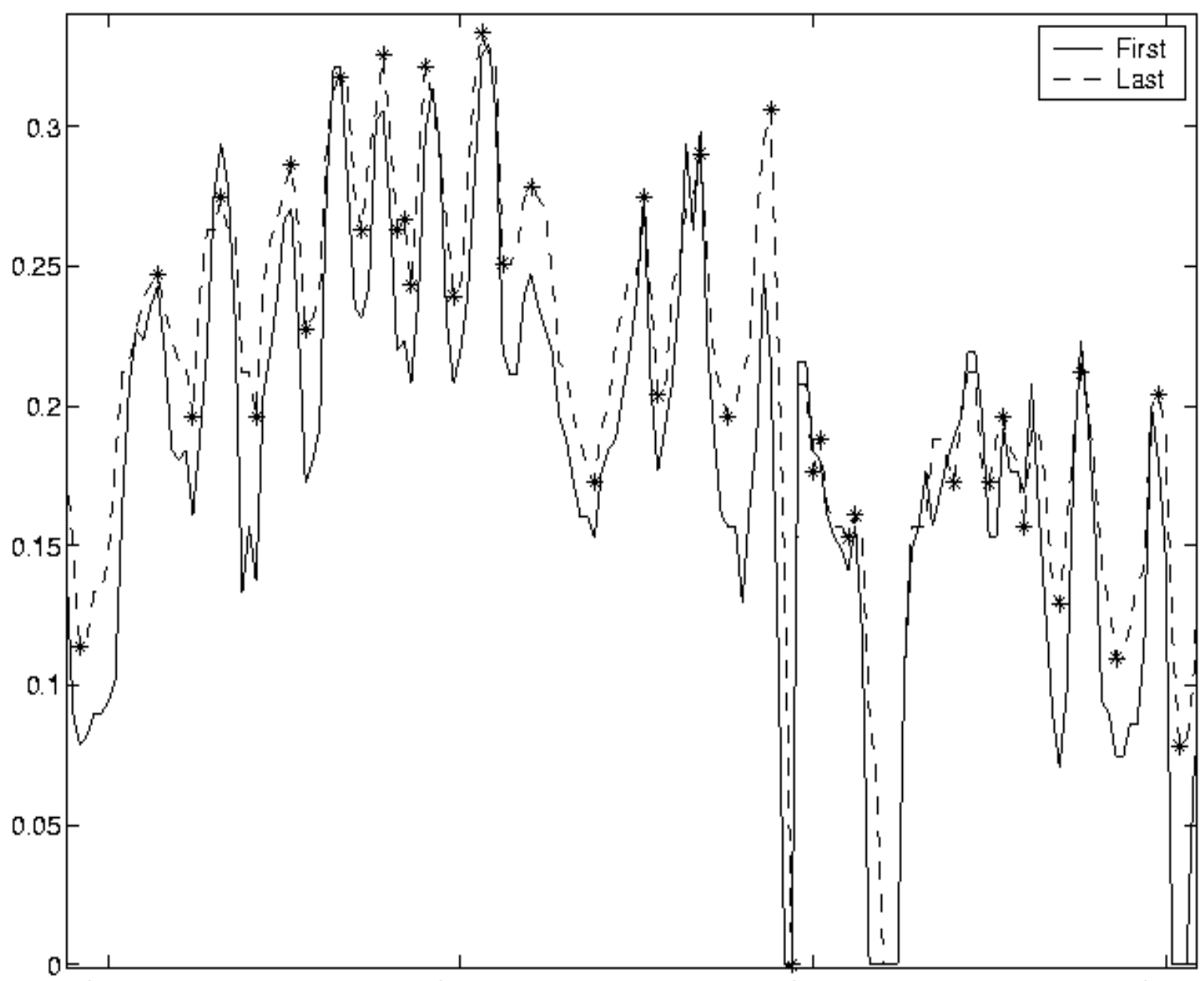

First versus last capture fingerprint signals for dynamic measures. Extremums denoted by 


\section{Extracted Parameters:}

First Mean $=0.1674$ Last Mean $=0.2005$

MeanPercentChange $=19.820$

FirstFFTstd $=4.3377 \quad$ LastFFTstd $=3.7021$

FirstFFTstdLPF $=4.5441$ LastFFTstdLPF $=3.8925$

First std $=0.0784$ Last std $=0.0655$ stdPercentChange $=-16.506$

Last-FirstDiffMean $=0.033$ Last-FirstDiffStd $=0.041$

Original: SumOfFirstMINS=122.7843 SumOfFirstMAXS=155.1098

SumOfLastMINS=154.1961 SumOfLastMAXS=176.2745

Original NORMALIZED:SumOfFirstMINS $=0.160084$

SumOfFirstMAXS $=0.171014$

SumOfLastMINS $=0.201038$ SumOfLastMAXS $=0.194349$

Filtered: SumOfFirstMINS $=103.3961$ SumOfFirstMAXS $=155.1171$

SumOfLastMINS=132.0024 SumOfLastMAXS=175.5296

Filtered NORMALIZED:SumOfFirstMINS $=0.132900$

SomeOfFirstMAXS $=0.199123$

SumOfLastMINS=0.169669 SumOfLastMAXS=0 225327

Original: FirstToLastMAXSgrowth=21.1647

FirstToLastMINSgrowth $=31.4118$

FirstMINStoMAXS sum=32.3255 LastMINStoMAXSsum=22.0784

Original NORMALIZED: FirstToLastMAXSgrowth=0.023335

First ToLastMINSgrowth $=0.040954$

FirstMINStoMAXS sum $=0.035640$ LastMINStoMAXSsum $=0.024342$

Filtered: FirstToLastMAXSgrowth $=20.4125$

FirstToLastMINSgrowth $=28.6062$

FirstMINStoMAXSsum=51.7209 LastMINStoMAXSsum=43.5272

Filtered NORMALIZED: FirstToLastMAXSgrowth=0.027169

First ToLastMINSgrowth $=0.040375$

FirstMINStoMAXS sum $=0.041496$ LastMINStoMAXSsum=0.028342

Original NORMALIZED: FirstTotalSwing=0.028114

LastTotalSwing $=0.021751$

Filtered NORMALIZED: FilteredFirstTotalSwing=0.022533

FilteredLastTotalSwing $=0.016841$

stdFFTMeanFirst $=0.4691 \quad$ FFTstdMeanLast $=0.3777$ 


\section{Case 4 Live}

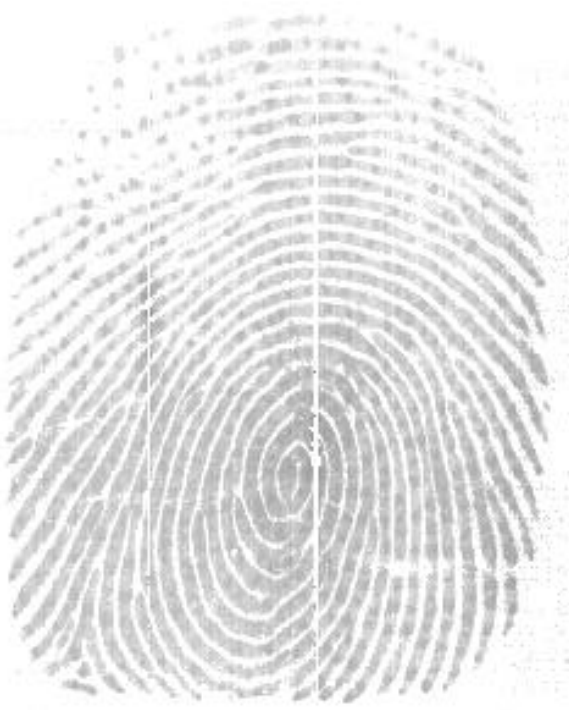

First Capture

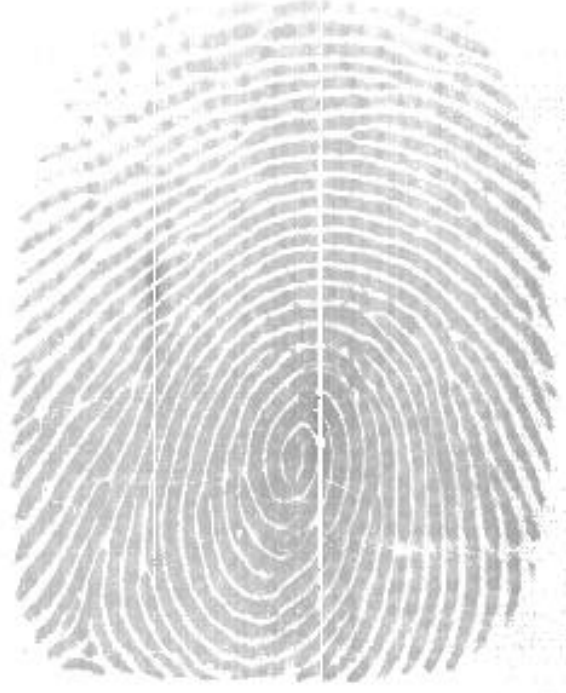

Last Capture

FFT of mean of first individual contours


FFT for Static Measure 


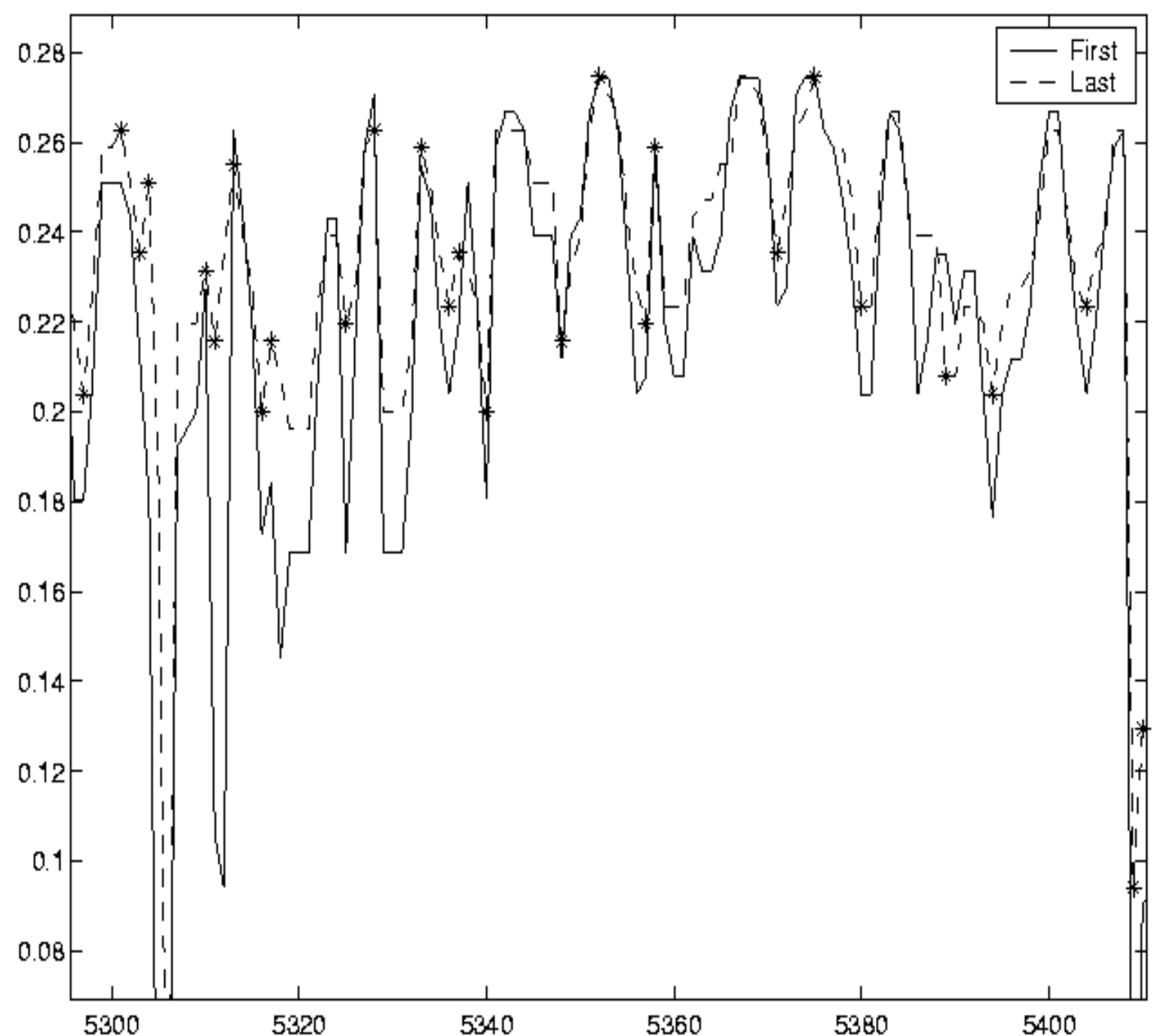

First versus last capture fingerprint signal, for dynamic measures. Extremums denoted by * 


\section{Extracted Parameters:}

First Mean $=0.1778$ Last Mean $=0.1995$

MeanPercentChange $=12.168$

FirstFFTstd $=4.1432$ LastFFTstd $=3.4667$

FirstFFTstdLPF $=4.3126$ LastFFTstdLPF $=3.5841$

First std $=0.0691$ Last std $=0.0568$ stdPercentChange $=-17.855$

Last $-F$ irstDiffMean $=0.022$ Last - FirstDiffStd $=0.025$

Original: SumOfFirstMINS=153.2196 SumOfFirstMAXS=159.4118

SumOfLastMINS=179.2784 SumOfLastMAXS=171.0941

Original NORMALIZED:SumOfFirstMINS=0.198471

SumOfFirstMAXS $=0.156593$

SumOfLastMINS $=0.232226$ SumOfLastMAXS $=0.168069$

Filtered: SumOfFirstMINS=132.7695 SumOfFirstMAXS=186.3953

SumOfLastMINS=156.5590 SumOfLastMAXS=199.1220

Filtered NORMALIZED:SumOfFirstMINS $=0.149347$

SomeOfFirstMAXS $=0.209433$

SumOfLastMINS $=0.176107$ SumOfLastMAXS $=0.223733$

Original: FirstToLastMAXSgrowth $=11.6824$

FirstToLastMINSgrowth $=26.0588$

FirstMINStoMAXSsum=6.1922 LastMINStoMAXSsum $=-8.1843$

Original NORMALIZED: FirstToLastMAXSgrowth=0.011476

FirstToLastMINSgrowth $=0.033755$

FirstMINStoMAXS sum $=0.006083$ LastMINStoMAXSsum $=-0.008040$

Filtered: FirstToLastMAXSgrowth=12.7266

FirstToLastMINSgrowth $=23.7895$

FirstMINStoMAXSsum $=53.6258$ LastMINStoMAXSsum $=42.5630$

Filtered NORMALIZED: FirstToLastMAXSgrowth $=0.013126$

FirstToLastMINSgrowth $=0.029313$

FirstMINStoMAXS sum $=0.006957$ LastMINStoMAXSsum $=-0.009196$

Original NORMALIZED: FirstTotalSwing=0.024255

Last Totalswing $=0.017953$

Filtered NORMALIZED: FilteredFirstTotalSwing=0.020901

FilteredLast TotalSwing $=0.015289$

stdFFTMeanFirst $=0.4015 \quad$ FFTstdMeanLast $=0.3080$ 


\section{Case 1 Spoof}

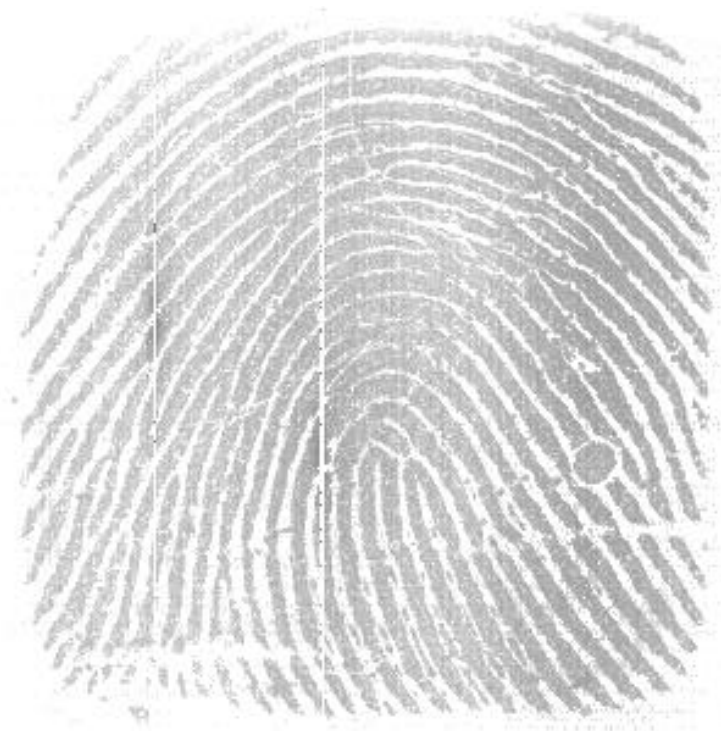

First Capture

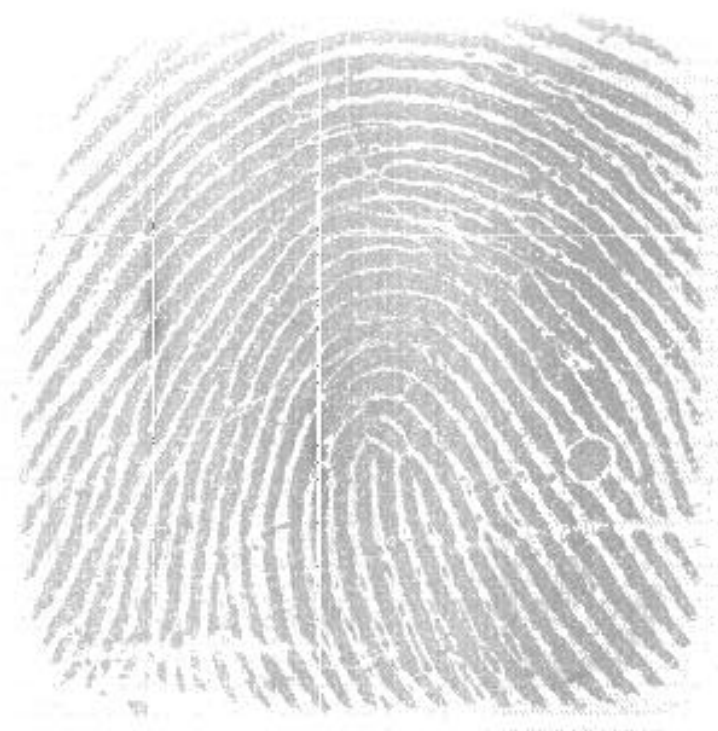

Last Capture
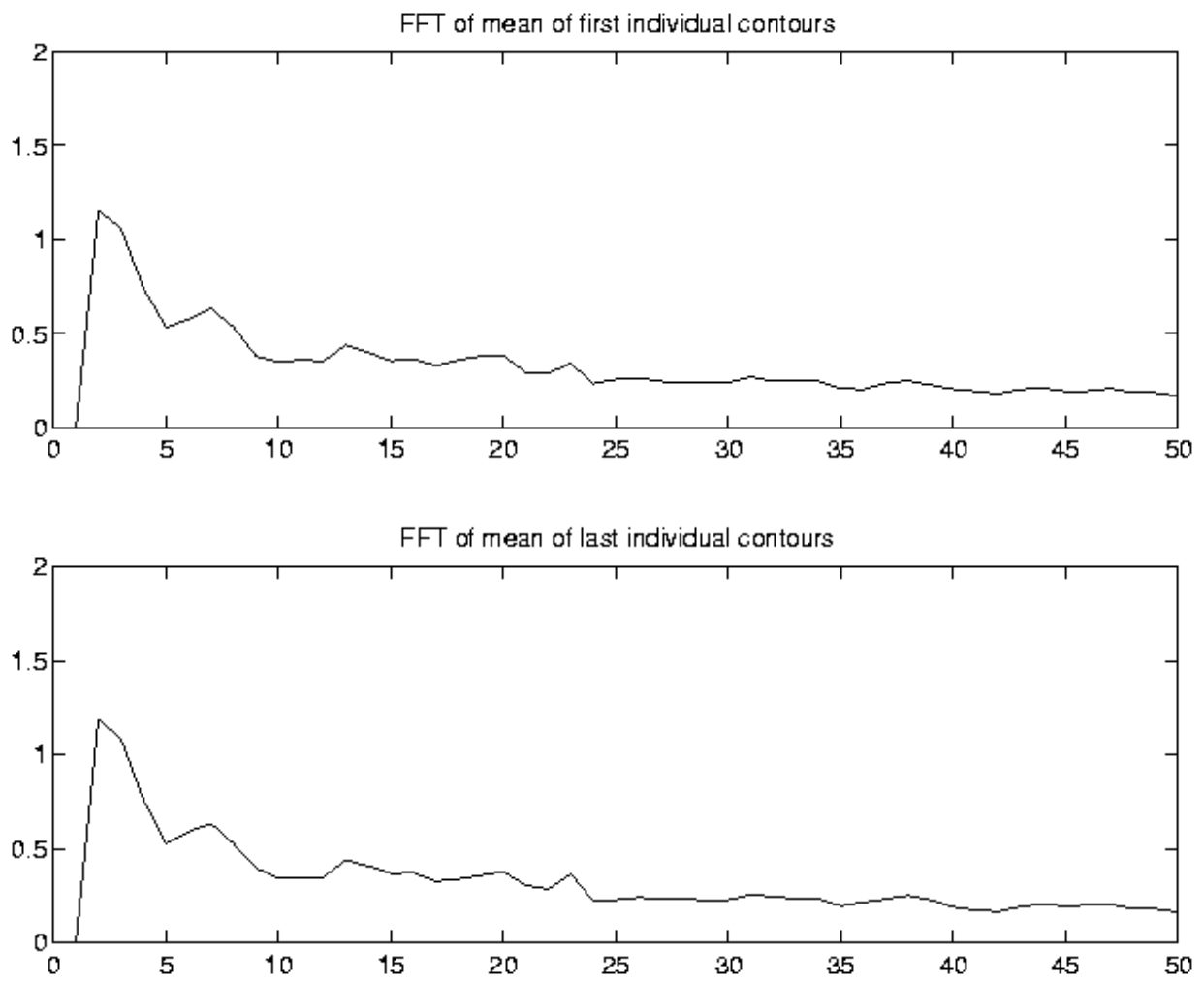

FFT for Static Measure 


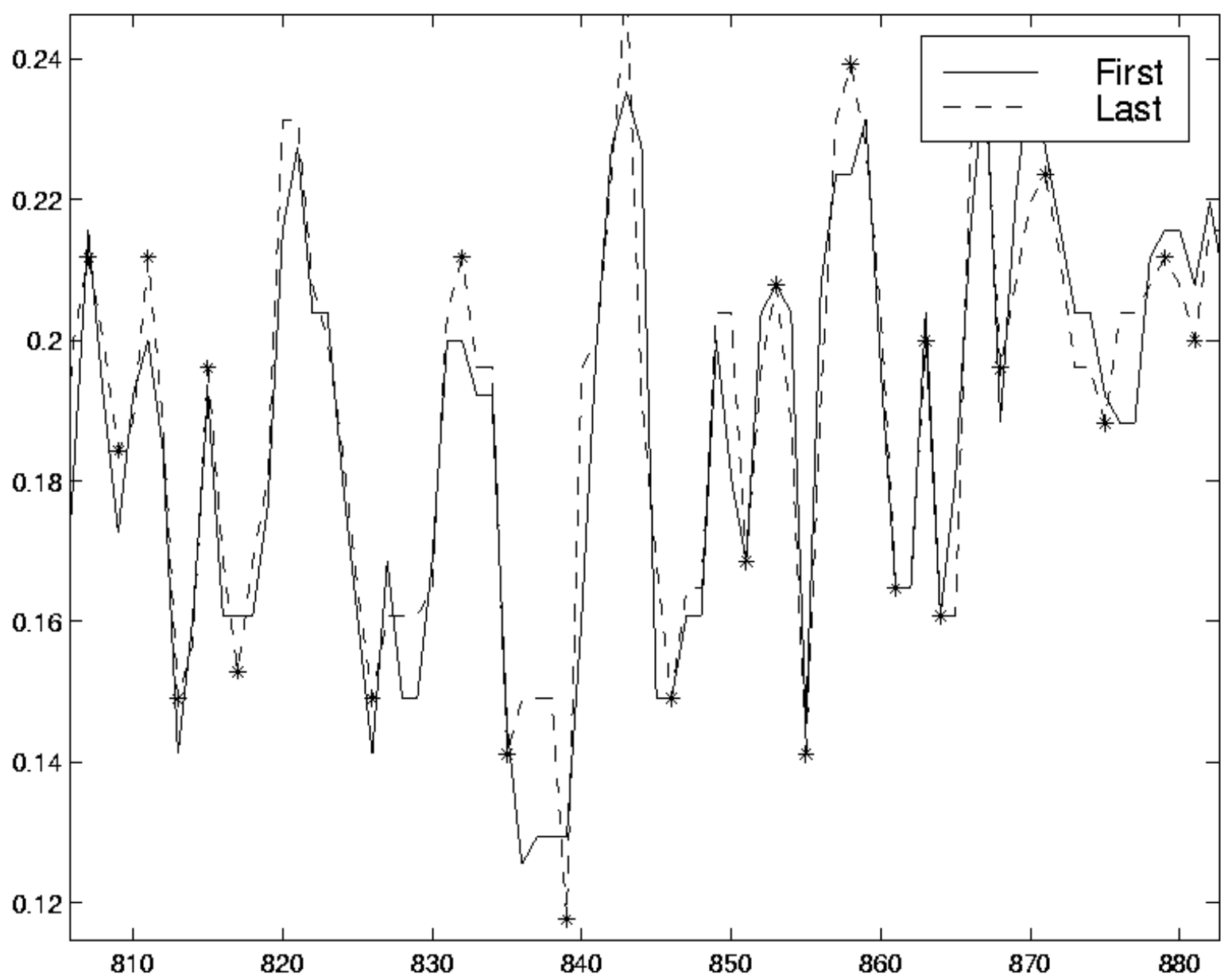

First versus last capture fingerprint signal, for dynamic measures. Extremums denoted by $*$ 


\section{Extracted Parameters}

First Mean $=0.2225$ Last Mean $=0.2278$

MeanPercentChange $=2.389$

FirstFFTstd $=3.7175$ LastFFTstd $=3.7878$

FirstFFTstdLPF $=3.8411$ LastFFTstdLPF $=3.9158$

First std $=0.0601$ Last std $=0.0610$ stdPercentChange $=1.450$

Last - FirstDiffMean $=0.005$ Last - FirstDiffStd $=0.011$

Original: SumOfFirstMINS $=240.8392$ SumOfFirstMAXS $=225.6471$

SumOfLastMINS=245.5059 SumOfLastMAXS=232.3647

Original NORMALIZED:SumOfFirstMINS $=0.255126$

SumOfFirstMAXS $=0.187726$

SumOfLastMINS $=0.260070 \quad$ SumOfLastMAXS $=0.193315$

Filtered: SumOfFirstMINS=200.3717 SumOfFirstMAXS=237.5797

SumOfLastMINS $=204.9034$ SumOfLastMAXS $=243.8506$

Filtered NORMALIZED:SumOfFirstMINS $=0.203011$

SomeOfFirstMAXS $=0.240709$

SumOfLastMINS $=0.207602$ SumOfLastMAXS $=0.247062$

Original: FirstToLastMAXSgrowth $=6.7176$

FirstToLastMINSgrowth $=4.6667$

FirstMINStoMAXSsum=-15.1922 LastMINStoMAXSsum=-13.1412

Original NORMALIZED: FirstToLastMAXSgrowth=0.005589

FirstToLastMINSgrowth $=0.004944$

FirstMINStoMAXS sum $=-0.012639$ LastMINStoMAXSsum $=-0.010933$

Filtered: FirstToLastMAXSgrowth $=6.2709$

FirstToLastMINSgrowth $=4.5318$

FirstMINStoMAXSsum=37.2081 LastMINStoMAXSsum=38.9472

Filtered NORMALIZED: FirstToLastMAXSgrowth=0.006806

FirstToLastMINSgrowth $=0.004728$

FirstMINStoMAXS sum $=-0.015392$ LastMINStoMAXSsum $=-0.013314$

Original NORMALIZED: FirstTotalSwing=0.019016

Last Totalswing $=0.018823$

Filtered NORMALIZED: FilteredFirstTotalSwing=0.014219

FilteredLast Totalswing $=0.013870$

stdFFTMeanFirst $=0.1607 \quad$ FFTstdMeanLast $=0.1648$ 


\section{Case 2 Spoof}

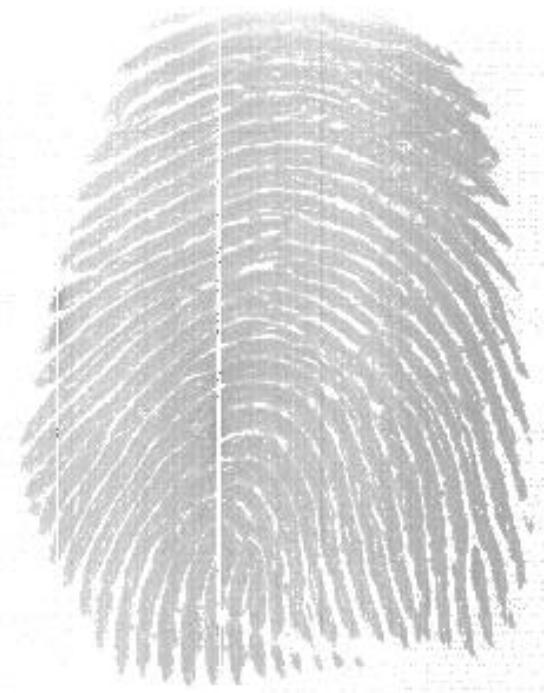

First Capture

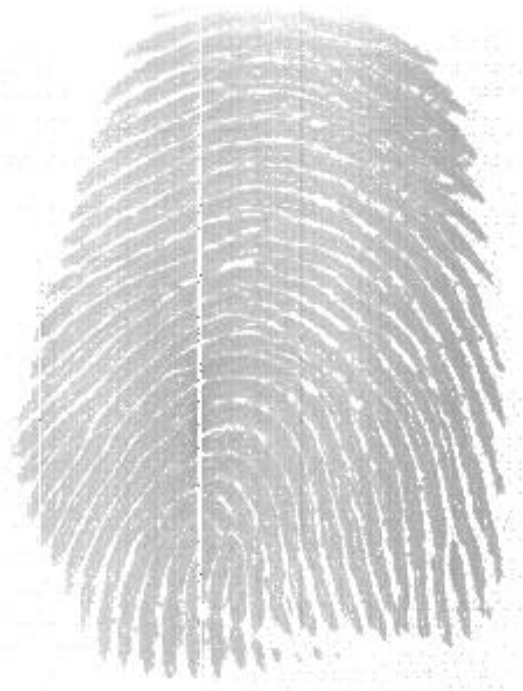

Last Capture
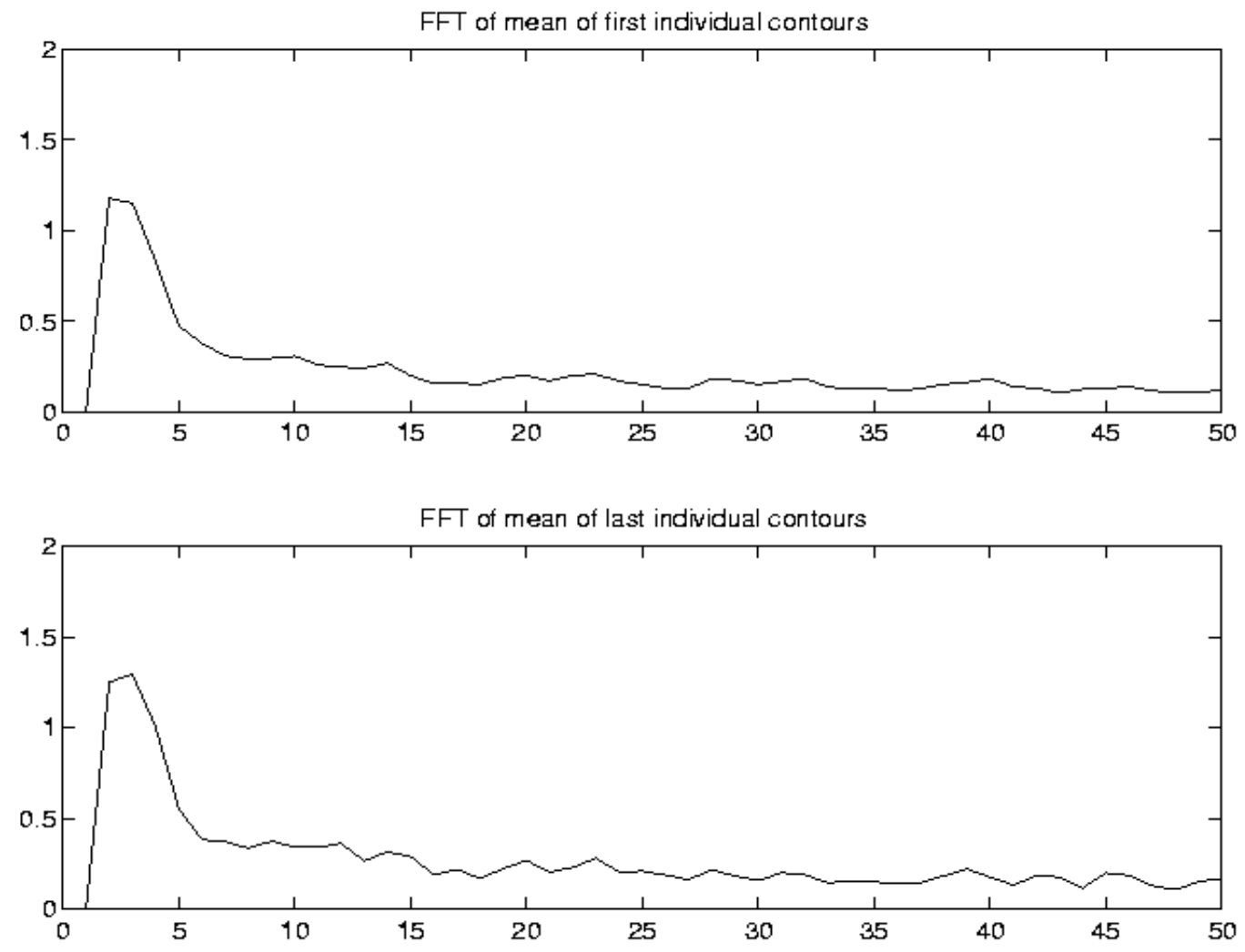

FFT for Static Measure 


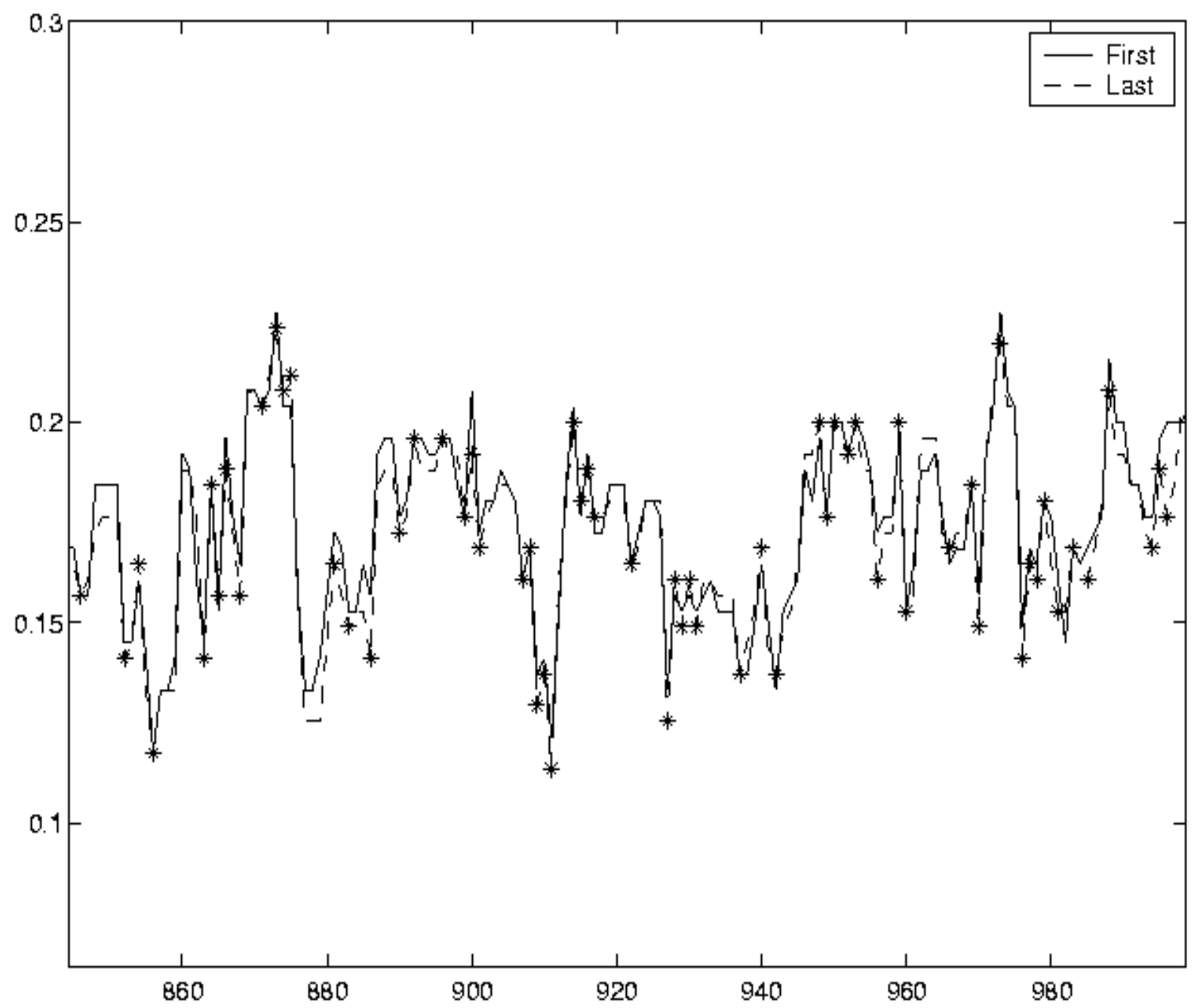

First versus last capture fingerprint signal, for dynamic measures. Extremums denoted by *. 


\section{Extracted Parameters}

First Mean=0.2197 Last Mean=0.2177 MeanPercentChange=0.908

FirstFFTstd $=2.4955$ LastFFTstd $=2.6565$

FirstFFTstdLPF $=2.5135$ LastFFTstdLPF $=2.6784$

First std $=0.0450$ Last std $=0.0491$ stdPercentChange $=9.320$ Last-FirstDiffMean $=-0.002$ Last-FirstDiffStd $=0.012$

Original: SumOfFirstMINS=157.9961 SumOfFirstMAXS=137.3137

SumOfLastMINS $=153.9098$ SumOfLastMAXS $=137.6431$

Original NORMALIZED:SumOfFirstMINS $=0.263327$

SumOfFirstMAXS $=0.180676$

SumOfLastMINS=0.256516 SumOfLastMAXS $=0.181109$

Filtered: SumOfFirstMINS $=142.7904$ SumOfFirstMAXS $=156.9033$

SumOfLastMINS $=139.8057$ SumOfLastMAXS=157.1549

Filtered NORMALIZED:SumOfFirstMINS $=0.207544$

SomeOfFirstMAXS $=0.228057$

SumOfLastMINS $=0.203206$ SumOfLastMAXS $=0.228423$

Original: FirstToLastMAXSgrowth $=0.3294$

FirstToLastMINSgrowth $=-4.0863$

FirstMINStoMAXS sum $=-20.6824$ LastMINStoMAXSsum $=-16.2667$

Original NORMALIZED: FirstToLastMAXSgrowth $=0.000433$

FirstToLastMINSgrowth $=-0.006810$

FirstMINStoMAXS sum $=-0.027214$ LastMINStoMAXSsum $=-0.021404$

Filtered: FirstToLastMAXSgrowth $=0.2516$

FirstToLastMINSgrowth $=-2.9847$

FirstMINStoMAXSsum=14.1129 LastMINStoMAXSsum=17.3492

Filtered NORMALIZED: FirstToLastMAXSgrowth $=0.000479$

FirstToLastMINSgrowth $=-0.005939$

FirstMINStoMAXSsum $=-0.030062$ LastMINStoMAXSsum $=-0.023643$

Original NORMALIZED: FirstTotalSwing=0.010429

Last Totalswing $=0.011540$

Filtered NORMALIZED: FilteredFirstTotalSwing=0.008076

FilteredLast TotalSwing=0.008936

stdFFTMeanFirst $=0.1575 \quad$ FFTstdMeanLast $=0.1766$ 


\section{Case 3 Spoof}

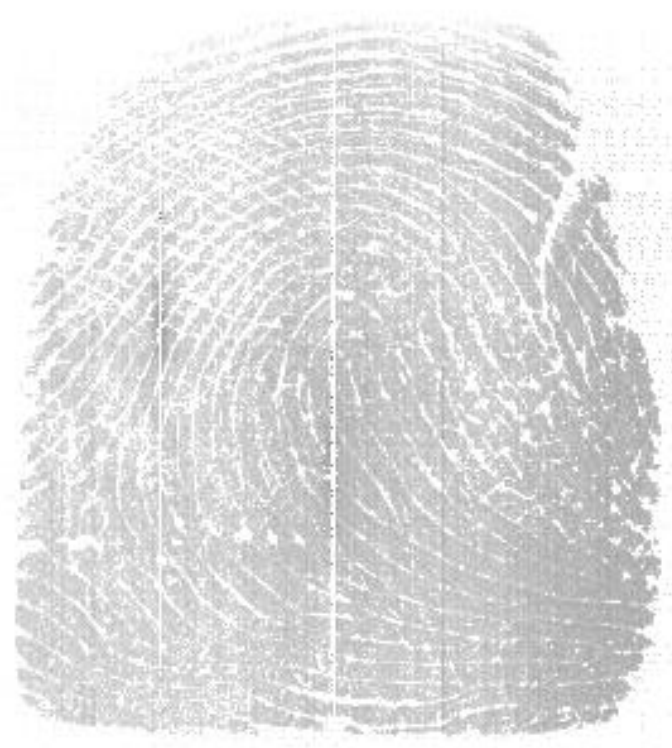

First Capture

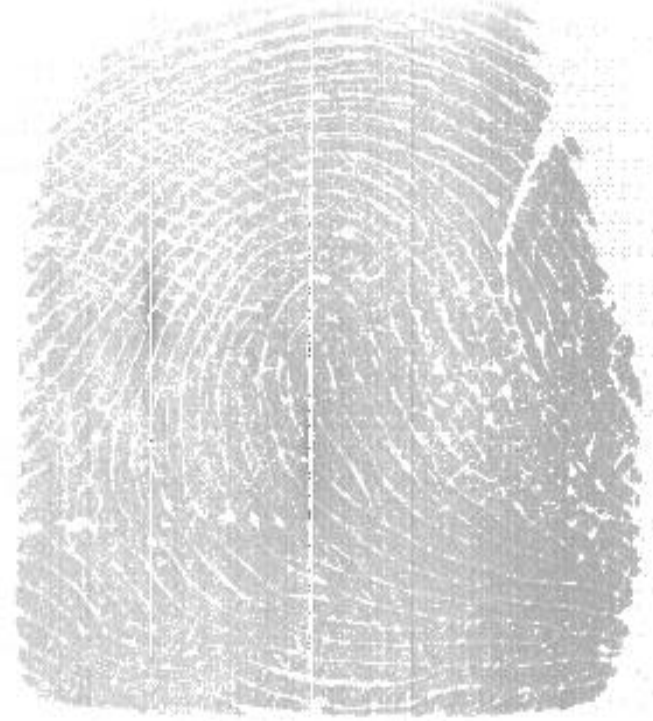

Last Capture

FFT of mean of first individual contours
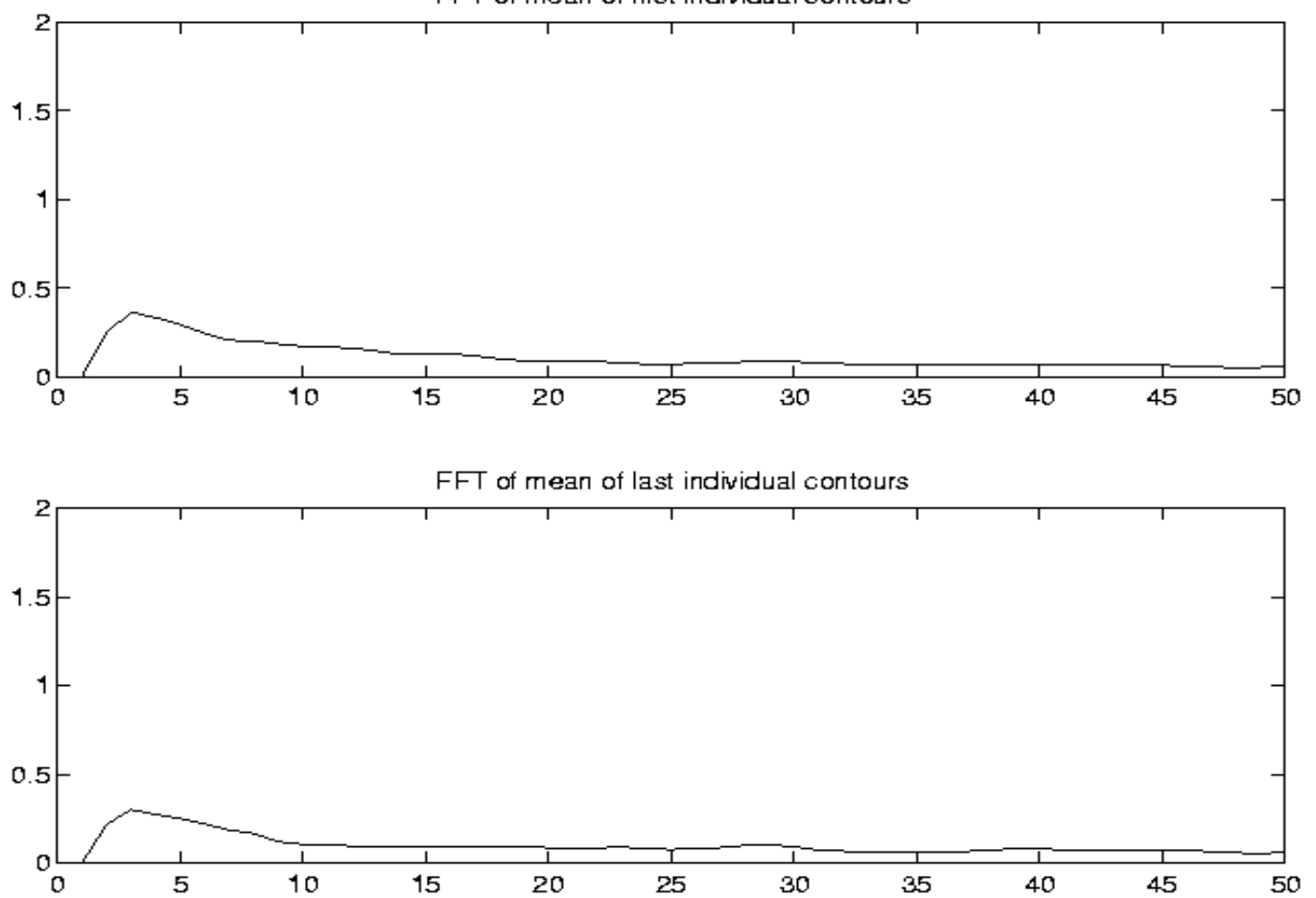

FFT for Static Measure 


\section{Extracted Parameters}

First Mean $=0.1705$ Last Mean $=0.1792$

MeanPercentChange $=5.080$

FirstFFTstd $=2.9538$ LastFFTstd $=2.7346$

FirstFFTstdLPF $=2.9836$ LastFFTstdLPF $=2.8060$

First std $=0.0605$ Last std $=0.0554$ stdPercentChange $=-8.448$

Last-FirstDiffMean=0.009 Last-FirstDiffStd=0.045

Original: SumOfFirstMINS=112.0667 SumOfFirstMAXS $=111.4588$

SumOfLastMINS $=116.8157$ SumOfLastMAXS $=117.1020$

Original NORMALIZED:SumOfFirstMINS=0.189622

SumOfFirstMAXS $=0.149209$

SumOfLastMINS $=0.197658$ SumOfLastMAXS $=0.156763$

Filtered:SumOfFirstMINS=92.4867 SumOfFirstMAXS=114.3212

SumOfLastMINS $=97.0701$ SumOfLastMAXS $=120.3899$

Filtered NORMALIZED:SumOfFirstMINS=0.151369

SomeOfFirstMAXS $=0.187105$

SumOfLastMINS=0.158871 SumOfLastMAXS=0.197037

Original: FirstToLastMAXSgrowth $=5.6431$

FirstToLastMINSgrowth $=4.7490$

FirstMINStoMAXS sum $=-0.6078$ LastMINStoMAXSsum $=0.2863$

Original NORMALIZED: FirstToLastMAXSgrowth $=0.007554$

FirstToLastMINSgrowth $=0.008036$

FirstMINStoMAXS sum $=-0.000814$ LastMINStoMAXS sum $=0.000383$

Filtered: FirstToLastMAXSgrowth $=6.0687$

FirstToLastMINSgrowth $=4.5834$

FirstMINStoMAXSsum=21.8345 LastMINStoMAXSsum=23.3198

Filtered NORMALIZED: FirstToLastMAXSgrowth $=0.009236$

FirstToLastMINSgrowth $=0.007773$

FirstMINStoMAXSsum $=-0.000995$ LastMINStoMAXSsum=0.000469

Original NORMALIZED: FirstTotalSwing=0.017560

LastTotalswing $=0.017317$

Filtered NORMALIZED: FilteredFirstTotalSwing=0.013769

FilteredLastTotalSwing $=0.013007$

stdFFTMeanFirst $=0.0600 \quad$ FFTstdMeanLast $=0.0474$ 


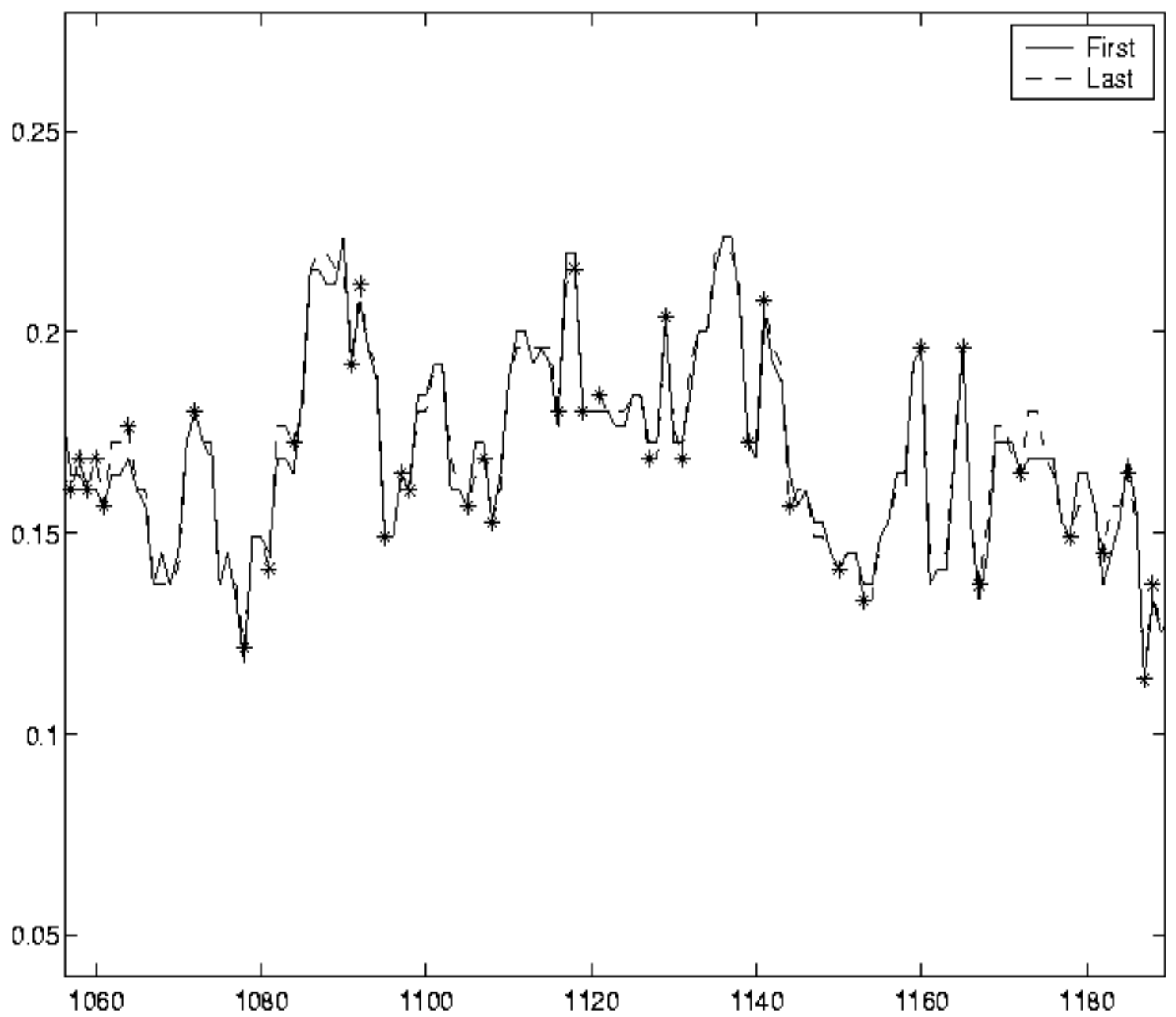

First versus last capture fingerprint signal, for dynamic measures. Extremums denoted by $*$ 


\section{Case 4 Spoof}

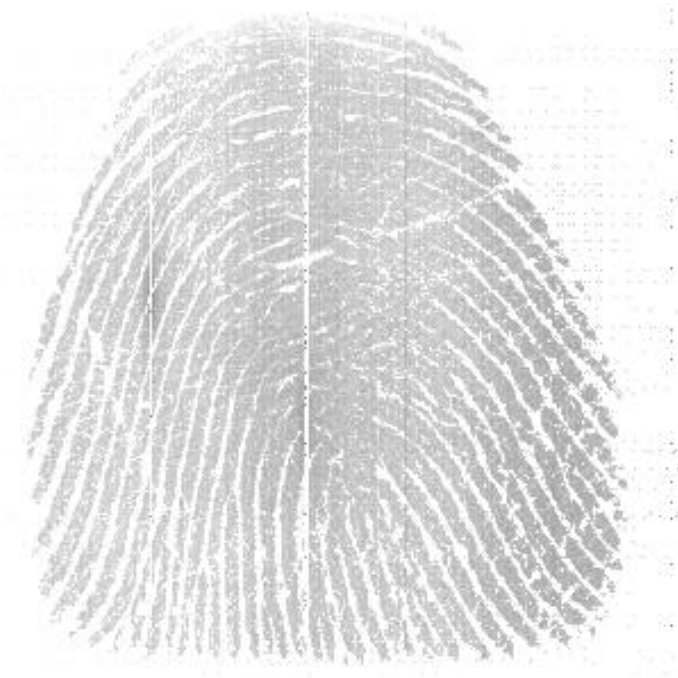

First Capture

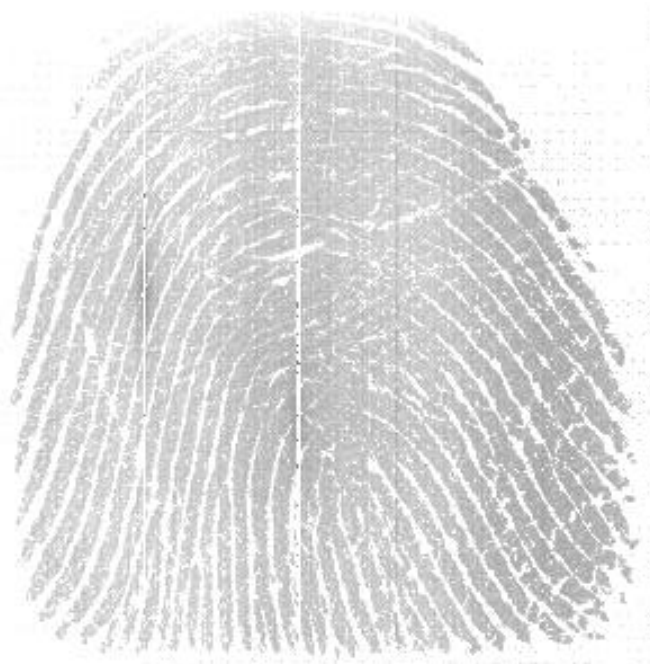

Last Capture
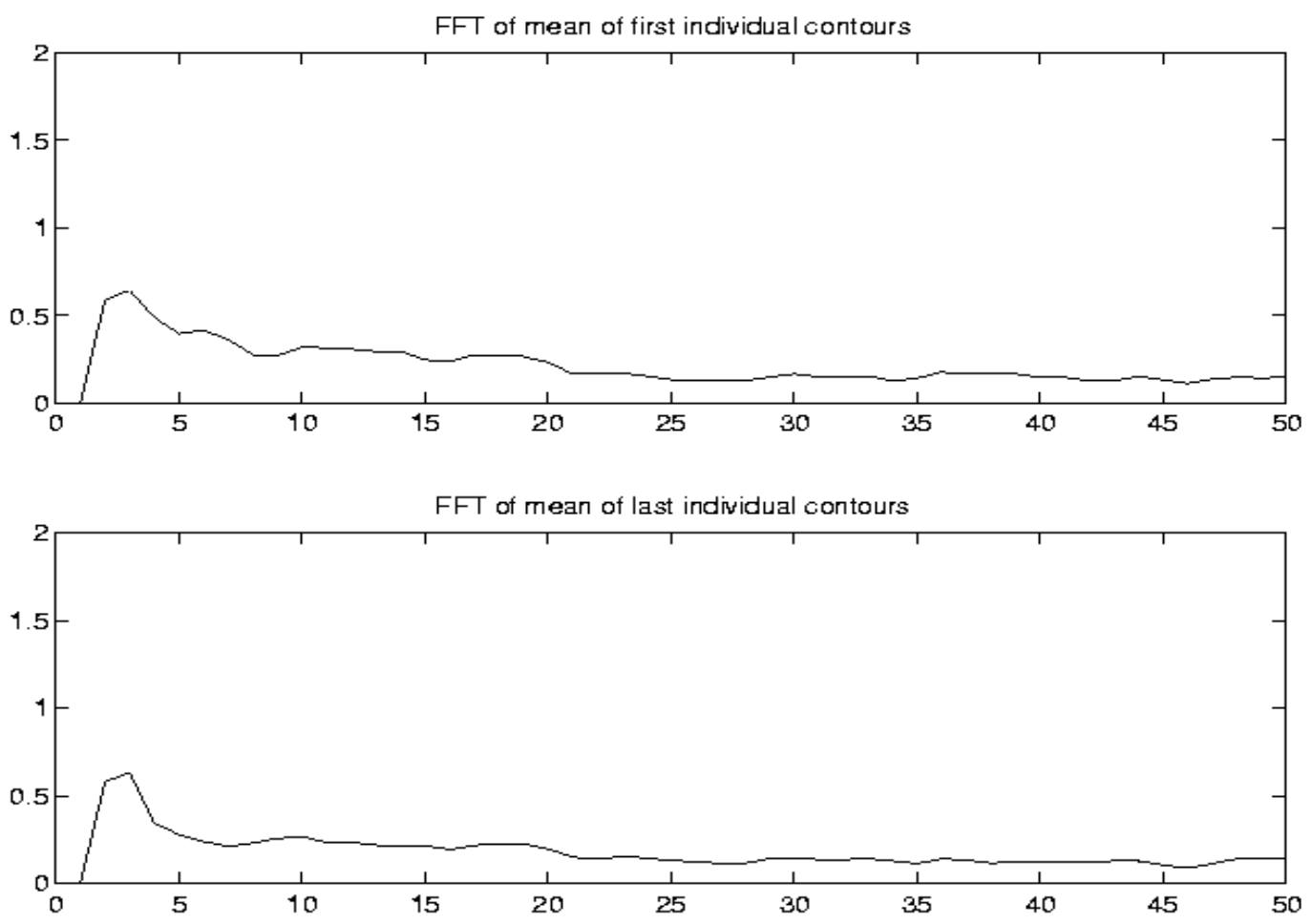

FFT for Static Measure 


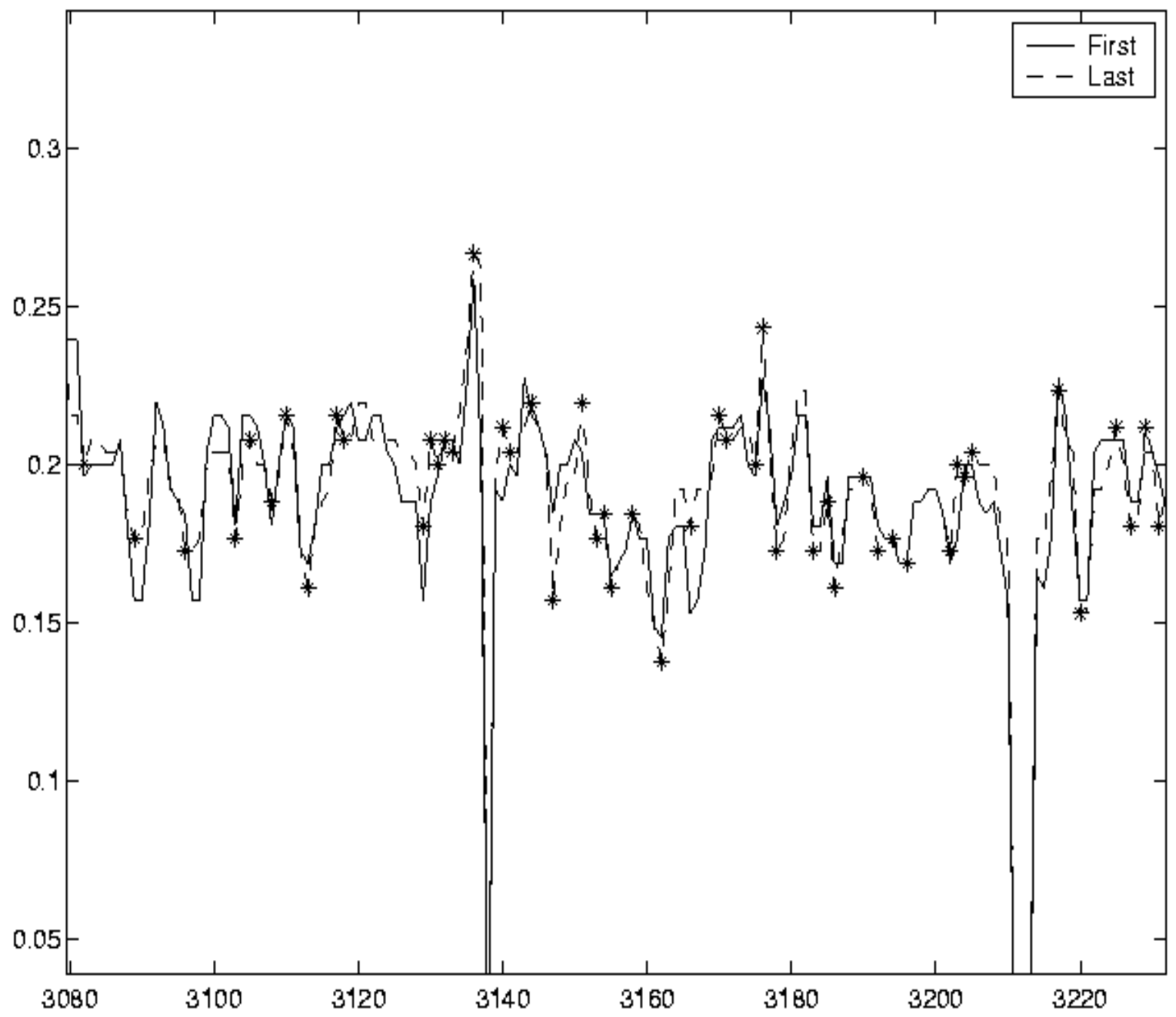

First versus last capture fingerprint signal, for dynamic measures. Extremums denoted by $*$ 


\section{Extracted Parameters}

First Mean $=0.1869$ Last Mean $=0.2031$

MeanPercentChange $=8.630$

FirstFFTstd $=3.9411$ LastFFTstd $=3.0747$

FirstFFTstdLPF $=4.0184$ LastFFTstdLPF $=3.1502$

First std $=0.0699$ Last std=0.0539 stdPercentChange $=-22.779$

Last-FirstDiffMean $=0.016$ Last $-F i r s t D i f f S t d=0.050$

Original: SumOfFirstMINS=161.9294 SumOfFirstMAXS=141.0196

SumOfLastMINS $=172.5490 \quad$ SumOfLastMAXS $=156.6549$

Original NORMALIZED:SumOfFirstMINS $=0.225528$

SumOfFirstMAXS $=0.148130$

SumOfLastMINS $=0.240319$ SumOfLastMAXS $=0.164553$

Filtered: SumOfFirstMINS $=137.3533$ SumOfFirstMAXS $=162.1654$

SumOfLastMINS $=148.0717$ SumOfLastMAXS $=177.4192$

Filtered NORMALIZED:SumOfFirstMINS $=0.170625$

SomeOfFirstMAXS $=0.201198$

SumOfLastMINS $=0.183940$ SumOfLastMAXS $=0.220123$

Original: FirstToLastMAXSgrowth=15.6353

FirstToLastMINSgrowth $=10.6196$

FirstMINStoMAXS sum $=-20.9098$ LastMINStoMAXSsum $=-15.8941$

Original NORMALIZED: FirstToLastMAXSgrowth=0.016424

First ToLastMINSgrowth $=0.014791$

FirstMINStoMAXSsum $=-0.021964$ LastMINStoMAXSsum $=-0.016696$

Filtered: FirstToLastMAXSgrowth $=15.2537$

FirstToLastMINSgrowth $=10.7184$

FirstMINStoMAXSsum=24.8122 LastMINStoMAXSsum=29.3475

Filtered NORMALIZED: FirstToLastMAXSgrowth=0.019399

FirstToLastMINSgrowth $=0.013192$

FirstMINStoMAXSsum $=-0.025943$ LastMINStoMAXSsum $=-0.019720$

Original NORMALIZED: FirstTotalSwing=0.018834

Last Totalswing $=0.016434$

Filtered NORMALIZED: FilteredFirstTotalSwing=0.015052

FilteredLast TotalSwing $=0.012742$

stdFFTMeanFirst $=0.1038$ FFTstdMeanLast $=0.0872$ 


\section{Case 1 Dead}

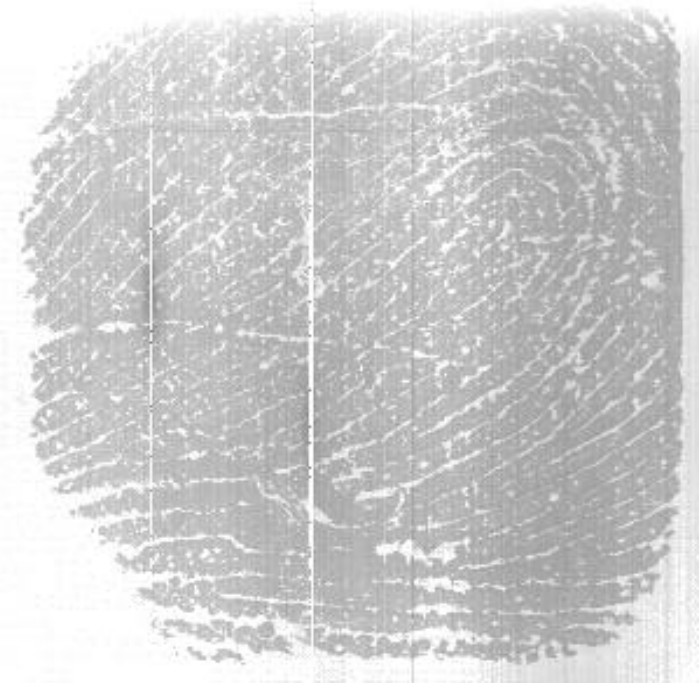

First Capture

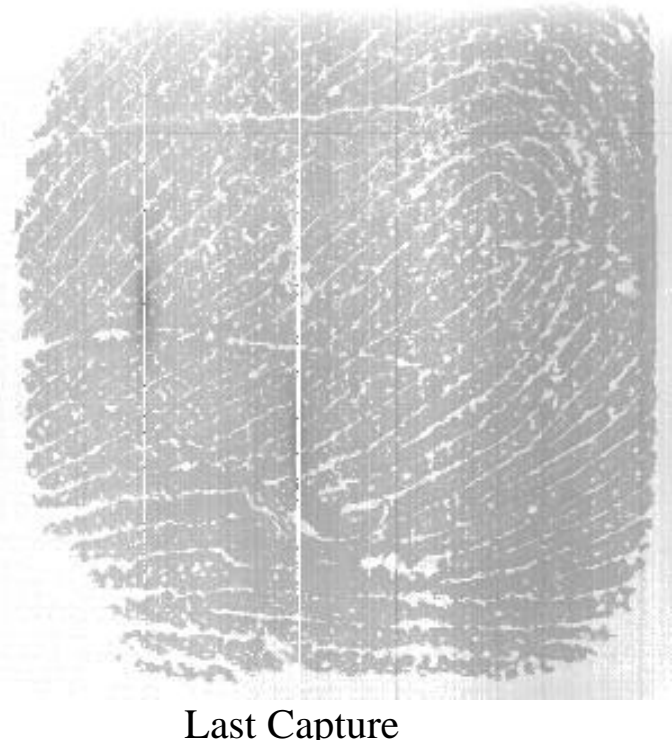

Last Capture

FFT of mean of first individual contours

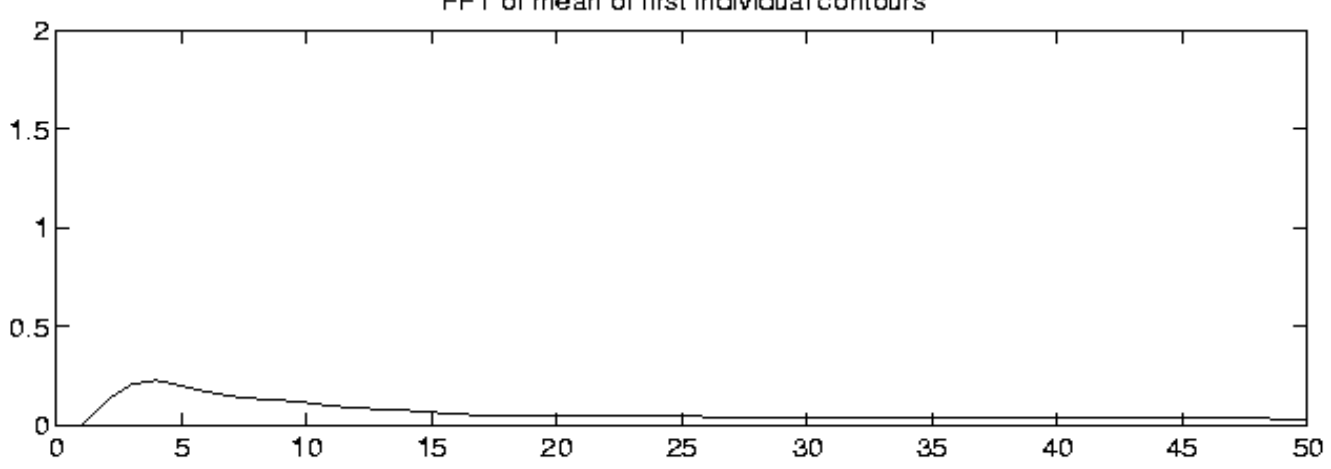

FFT of mean of last individual contours

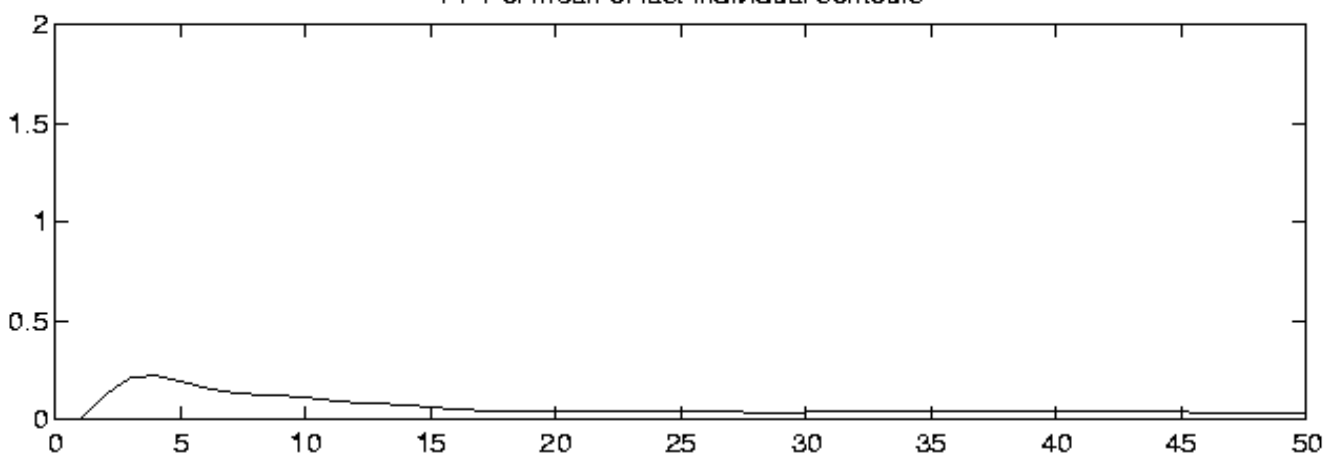

FFT for Static Measure 


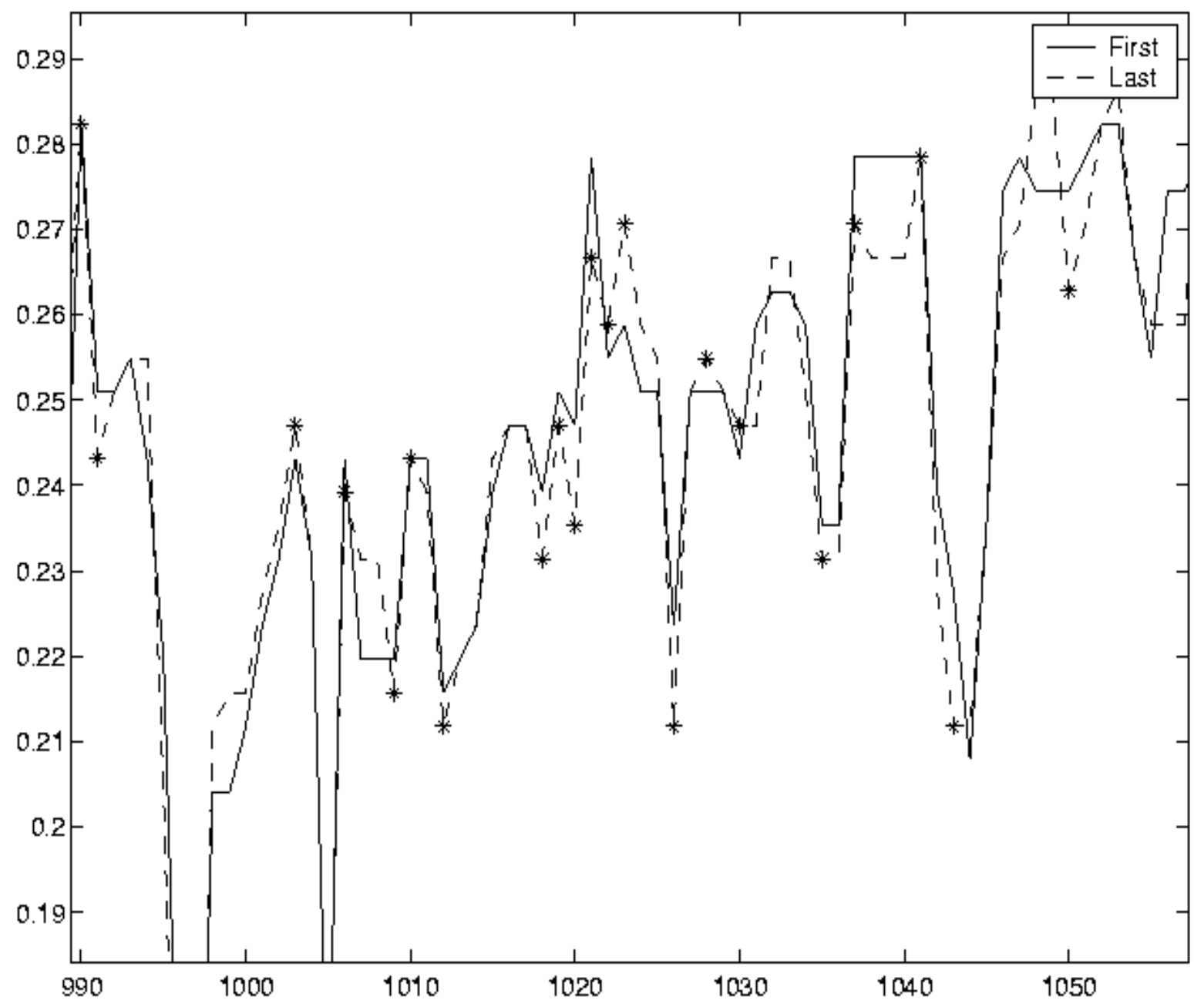

First versus last capture fingerprint signal, for dynamic measures. Extremums denoted by $*$ 


\section{Extracted Parameters}

First Mean $=0.2566$ Last Mean $=0.2557$ MeanPercentChange $=-$ 0.334

FirstFFTstd $=2.4909$ LastFFTstd $=2.5959$

FirstFFTstdLPF $=2.5048$ LastFFTstdLPF $=2.6172$

First std $=0.0550$ Last $s t d=0.0562$ stdPercentChange $=2.030$ Last-FirstDiffMean=-0.001 Last-FirstDiffStd $=0.019$

Original: SumOfFirstMINS $=129.7098$ SumOfFirstMAXS $=102.7882$

SumOfLastMINS $=128.1765$ SumOfLastMAXS $=103.5647$

Original NORMALIZED:SUmOfFirstMINS=0.336909

SumOfFirstMAXS $=0.191056$

SumOfLastMINS $=0.332926$ SumOfLastMAXS $=0.192499$

Filtered: SumOfFirstMINS=116.4516 SumOfFirstMAXS=129.7121

SumOfLastMINS $=115.4057$ SumOfLastMAXS $=129.8163$

Filtered NORMALIZED: SumOfFirstMINS $=0.243623$

SomeOfFirstMAXS $=0.271364$

SumOfLastMINS $=0.241435$ SumOfLastMAXS $=0.271582$

Original: FirstToLastMAXSgrowth $=0.7765$

FirstToLastMINSgrowth $=-1.5333$

FirstMINStoMAXSsum $=-26.9216$ LastMINSt oMAXS sum $=-24.6118$

Original NORMALIZED: FirstToLastMAXSgrowth=0.001443

FirstToLastMINSgrowth $=-0.003983$

FirstMINSt OMAXS sum $=-0.050040$ LastMINSt oMAXS sum $=-0.045747$

Filtered: FirstToLastMAXSgrowth $=0.1043$

FirstToLastMINSgrowth $=-1.0459$

FirstMINStoMAXS sum=13.2605 LastMINStoMAXSsum $=14.4106$

Filtered NORMALIZED: FirstToLastMAXSgrowth $=0.001624$

FirstToLastMINSgrowth $=-0.003208$

FirstMINSt OMAXS sum $=-0.056321$ LastMINSt oMAXS sum $=-0.051489$

Original NORMALIZED: FirstTotalSwing $=0.013794$

LastTotalSwing $=0.012888$

Filtered NORMALIZED: FilteredFirstTotalSwing $=0.011433$

FilteredLastTotalSwing $=0.010458$

stdFFTMeanFirst $=0.0388$ FFTstdMeanLast $=0.0374$ 


\section{Case 2 Dead}

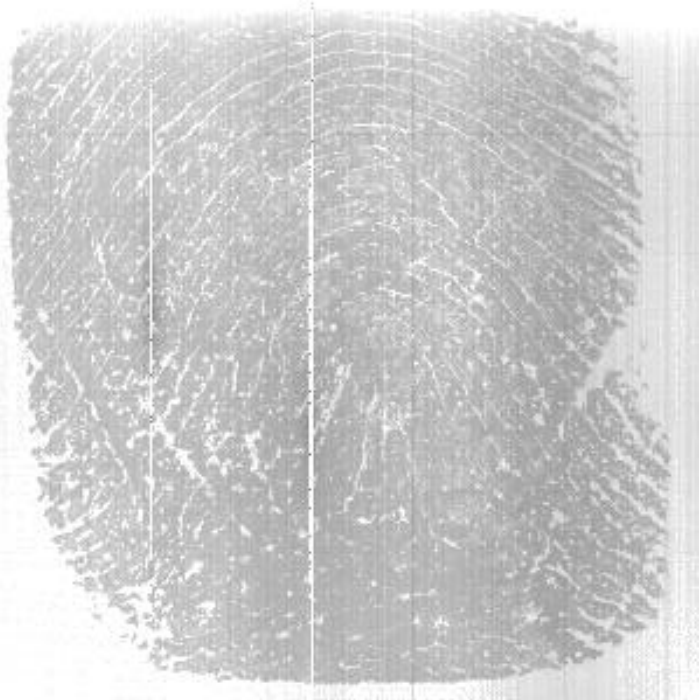

First Capture

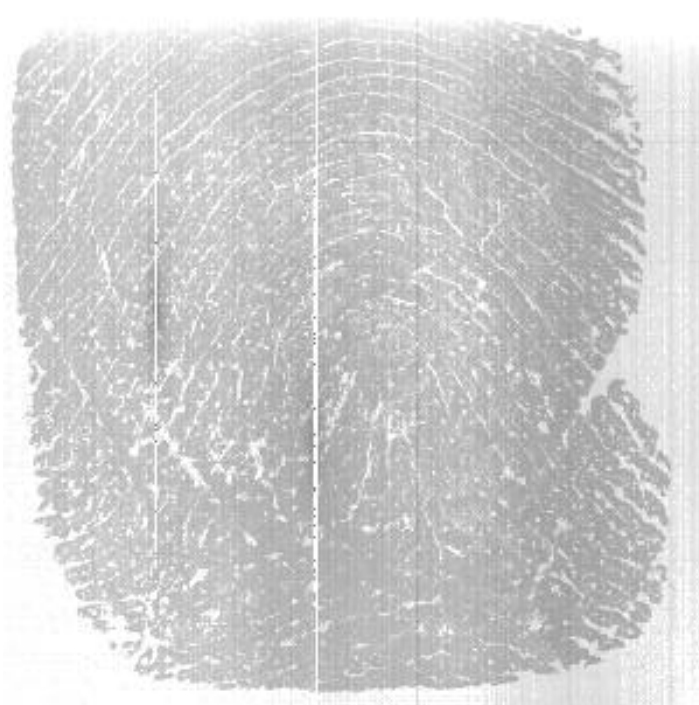

Last Capture

FFT of mean of first individual contours

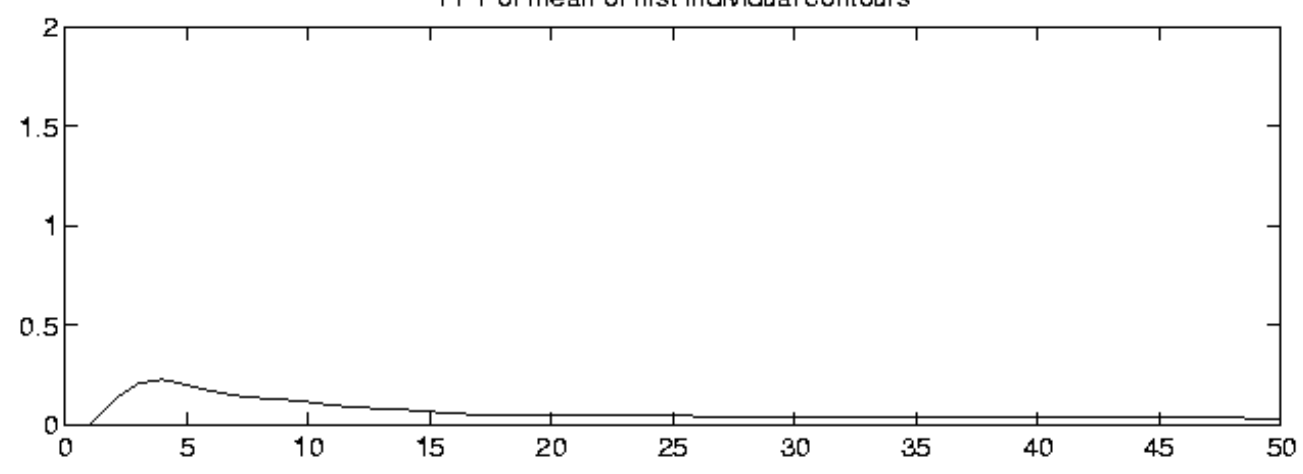

FFT of mean of last individual contours

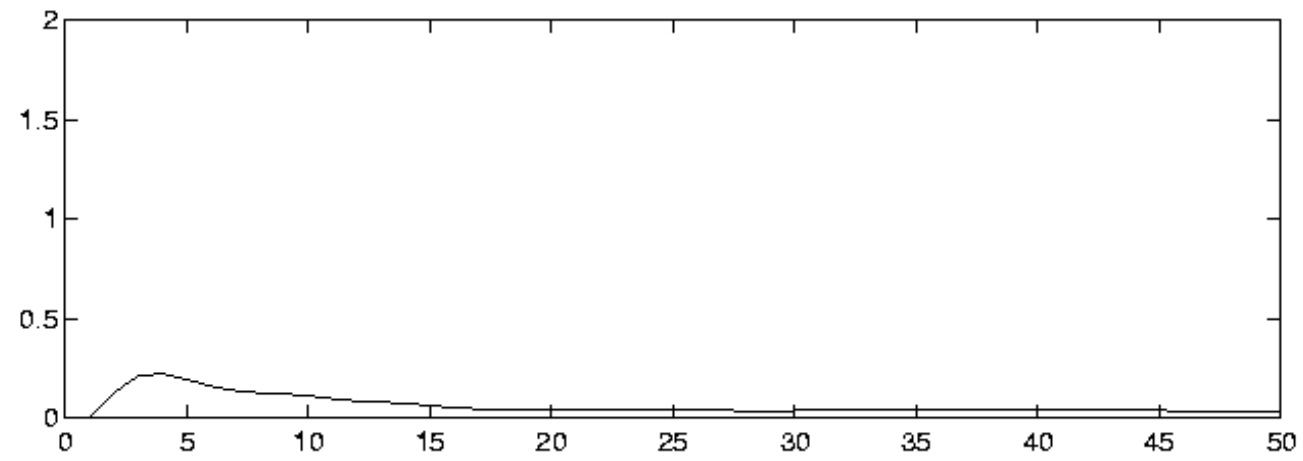

FFT for Static Measure 


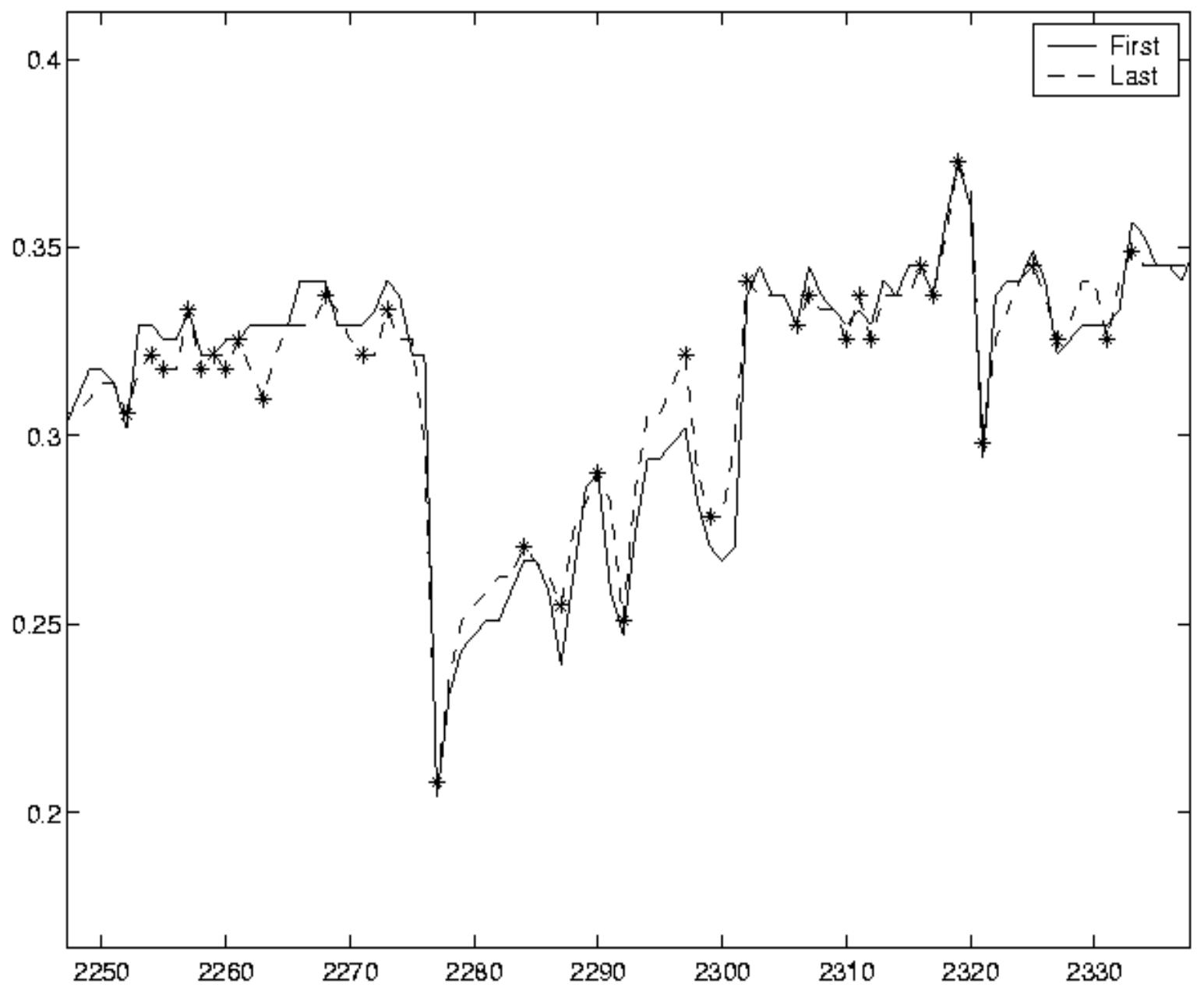

First versus last capture fingerprint signal, for dynamic measures. Extremums denoted by $*$ 


\section{Extracted Parameters}

First Mean $=0.2566$ Last Mean=0.2557 MeanPercentChange=0.334

FirstFFTstd=2.4909 LastFFTstd=2.5959

FirstFFTstdLPF $=2.5048$ LastFFTstdLPF $=2.6172$

First std $=0.0550$ Last std $=0.0562$ stdPercentChange $=2.030$ Last-FirstDiffMean=-0.001 Last-FirstDiffStd=0.019

Original: SumOfFirstMINS $=129.7098$ SumOfFirstMAXS $=102.7882$

SumOfLastMINS $=128.1765$ SumOfLastMAXS=103.5647

Original NORMALIZED:SumOfFirstMINS $=0.336909$

SumOfFirstMAXS $=0.191056$

SumOfLastMINS $=0.332926$ SumOfLastMAXS $=0.192499$

Filtered: SumOfFirstMINS $=116.4516$ SumOfFirstMAXS $=129.7121$

SumOfLastMINS $=115.4057$ SumOfLastMAXS $=129.8163$

Filtered NORMALIZED:SumOfFirstMINS $=0.243623$

SomeOfFirstMAXS $=0.271364$

SumOfLastMINS=0.241435 SumOfLastMAXS $=0.271582$

Original: FirstToLastMAXSgrowth $=0.7765$

FirstToLastMINSgrowth $=-1.5333$

FirstMINStoMAXS sum $=-26.9216$ LastMINStoMAXS sum $=-24.6118$

Original NORMALIZED: FirstToLastMAXSgrowth=0.001443

FirstToLastMINSgrowth $=-0.003983$

FirstMINStoMAXSsum $=-0.050040$ LastMINStoMAXSsum $=-0.045747$

Filtered: FirstToLastMAXSgrowth=0.1043

FirstToLastMINSgrowth $=-1.0459$

FirstMINStoMAXS sum=13.2605 LastMINStoMAXSsum=14.4106

Filtered NORMALIZED: FirstToLastMAXSgrowth $=0.001624$

FirstToLastMINSgrowth $=-0.003208$

FirstMINStoMAXS sum $=-0.056321$ LastMINStoMAXSsum $=-0.051489$

Original NORMALIZED: FirstTotalSwing=0.013794

LastTotalswing $=0.012888$

Filtered NORMALIZED: FilteredFirstTotalSwing=0.011433

FilteredLastTotalSwing $=0.010458$

stdFFTMeanFirst $=0.0388$ FFTstdMeanLast $=0.0374$ 
Case 3 Dead

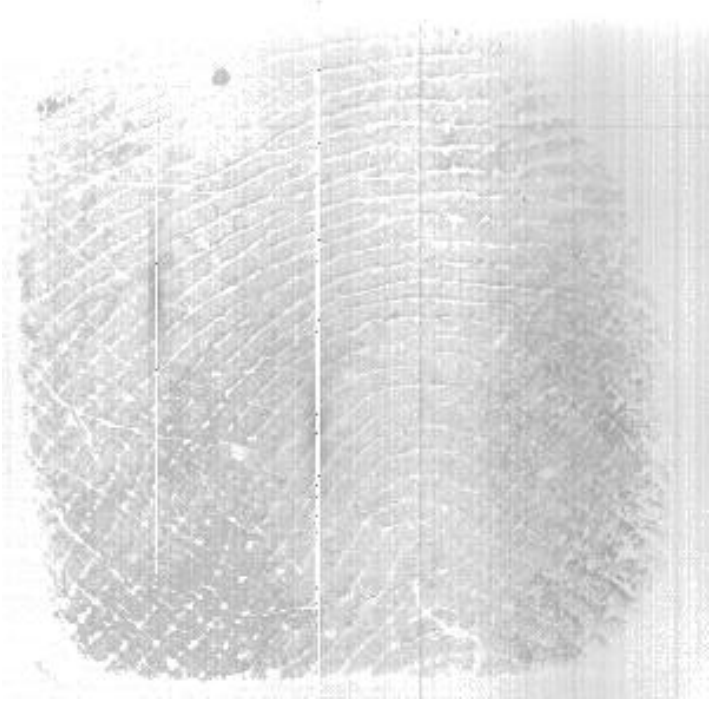

First Capture

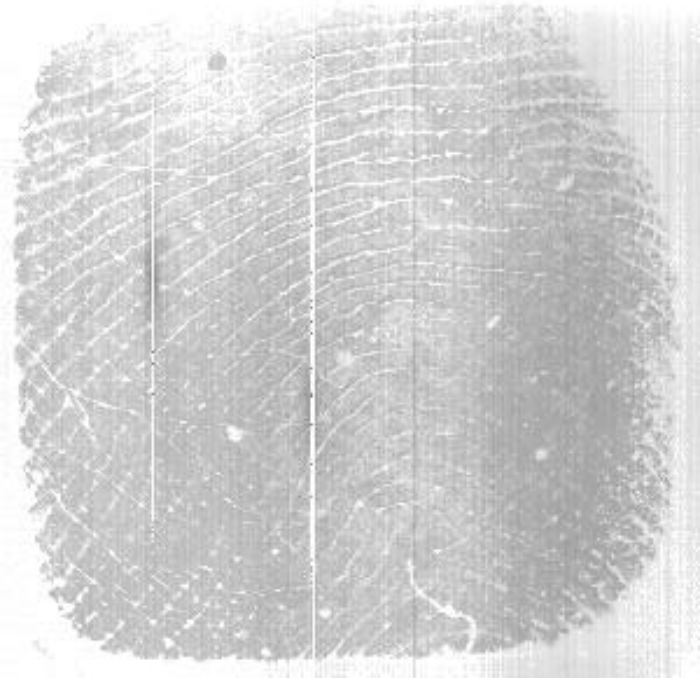

Last Capture

FFT of mean of first individual contours

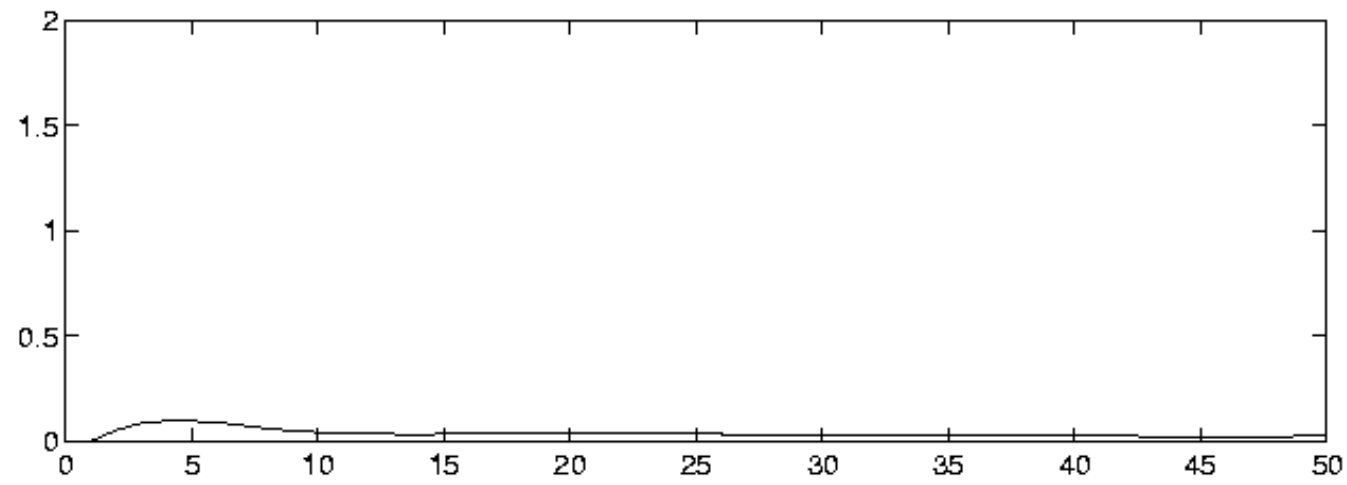

FFT of mean of last individual contours

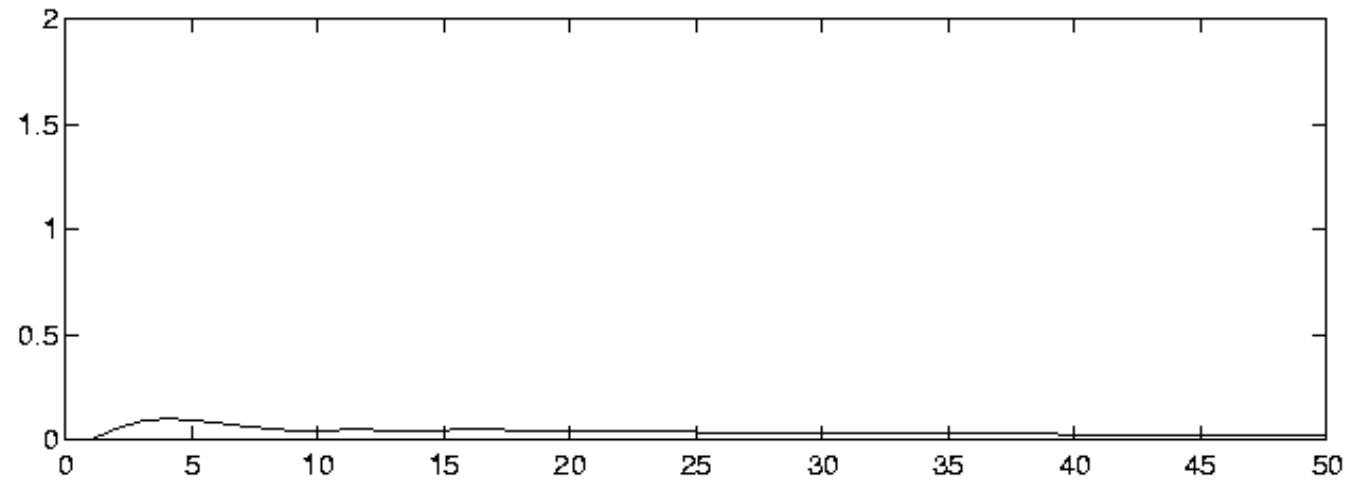

FFT for Static Measure 


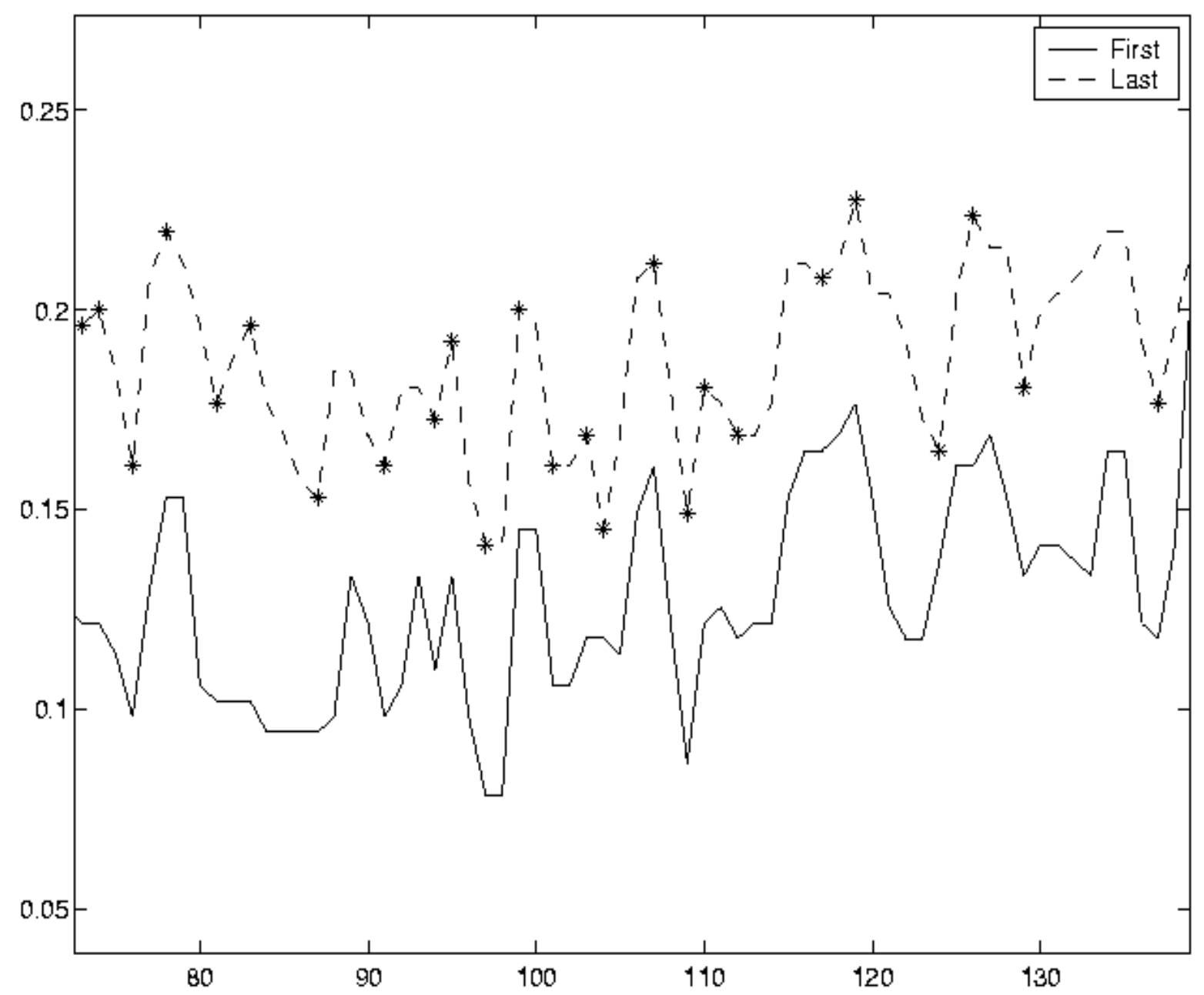

First versus last capture fingerprint signal, for dynamic measures. Extremums denoted by $*$ 


\section{Extracted Parameters}

First Mean $=0.2566$ Last Mean $=0.2557$ MeanPercentChange $=-$ 0.334

FirstFFTstd $=2.4909$ LastFFTstd $=2.5959$

FirstFFTstdLPF $=2.5048$ LastFFTstdLPF $=2.6172$

First std $=0.0550$ Last $s t d=0.0562$ stdPercentChange $=2.030$

Last-FirstDiffMean=-0.001 Last-FirstDiffStd=0.019

Original: SumOfFirstMINS=129.7098 SumOfFirstMAXS=102.7882

SumOfLastMINS $=128.1765$ SumOfLastMAXS $=103.5647$

Original NORMALIZED:SumOfFirstMINS=0.336909

SumOfFirstMAXS $=0.191056$

SumOfLastMINS $=0.332926$ SumOfLastMAXS $=0.192499$

Filtered: SumOfFirstMINS=116.4516 SumOfFirstMAXS=129.7121

SumOfLastMINS $=115.4057$ SumOfLastMAXS $=129.8163$

Filtered NORMALIZED: SumOfFirstMINS $=0.243623$

SomeOfFirstMAXS $=0.271364$

SumOfLastMINS=0.241435 SumOfLastMAXS=0.271582

Original: FirstToLastMAXSgrowth $=0.7765$

FirstToLastMINSgrowth $=-1.5333$

FirstMINStoMAXS sum $=-26.9216$ LastMINStoMAXSsum $=-24.6118$

Original NORMALIZED: FirstToLastMAXSgrowth $=0.001443$

FirstToLastMINSgrowth $=-0.003983$

FirstMINStoMAXSsum $=-0.050040$ LastMINStoMAXSsum $=-0.045747$

Filtered: FirstToLastMAXSgrowth $=0.1043$

FirstToLastMINSgrowth $=-1.0459$

FirstMINStoMAXSsum=13.2605 LastMINStoMAXSsum=14.4106

Filtered NORMALIZED: FirstToLastMAXSgrowth=0.001624

FirstToLastMINSgrowth $=-0.003208$

FirstMINStoMAXSsum $=-0.056321$ LastMINStoMAXSsum $=-0.051489$

Original NORMALIZED: FirstTotalSwing=0.013794

LastTotalswing $=0.012888$

Filtered NORMALIZED: FilteredFirstTotalSwing=0.011433

FilteredLastTotalSwing $=0.010458$

stdFFTMeanFirst $=0.0388$ FFTstdMeanLast $=0.0374$ 


\section{Case 4 Dead}

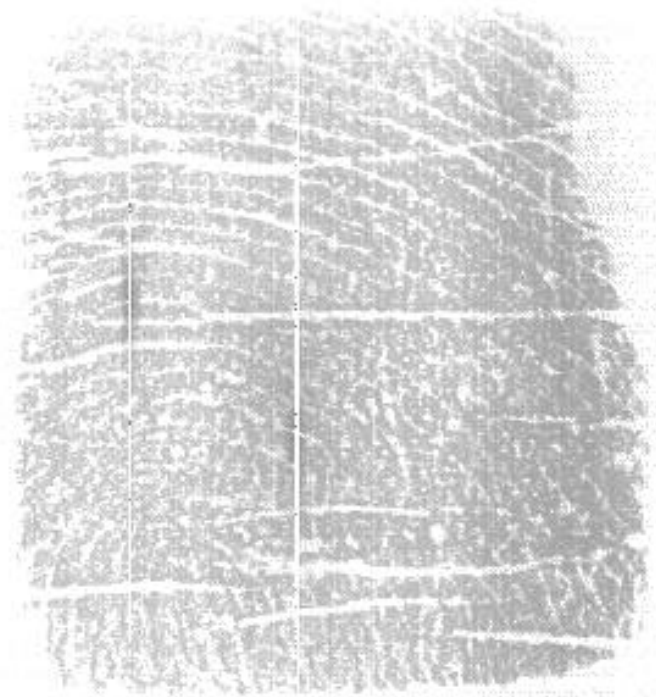

First Capture

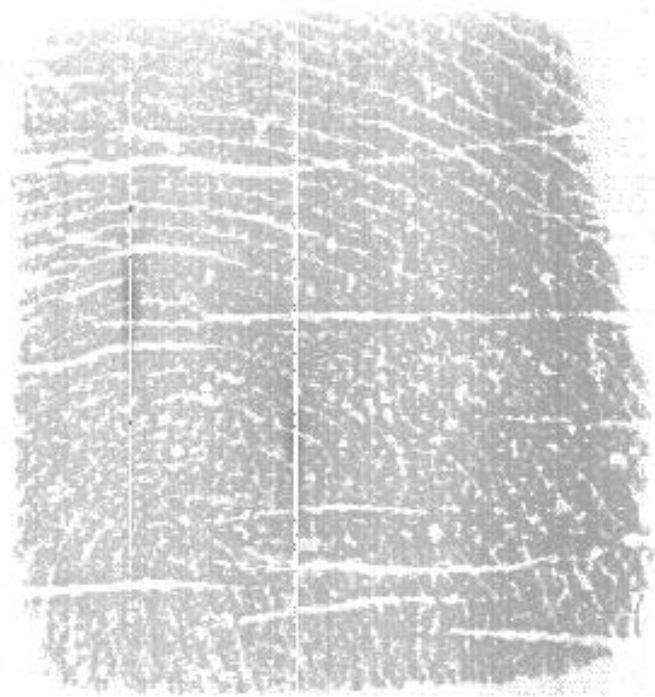

Last Capture

FFT of mean of first individual contours
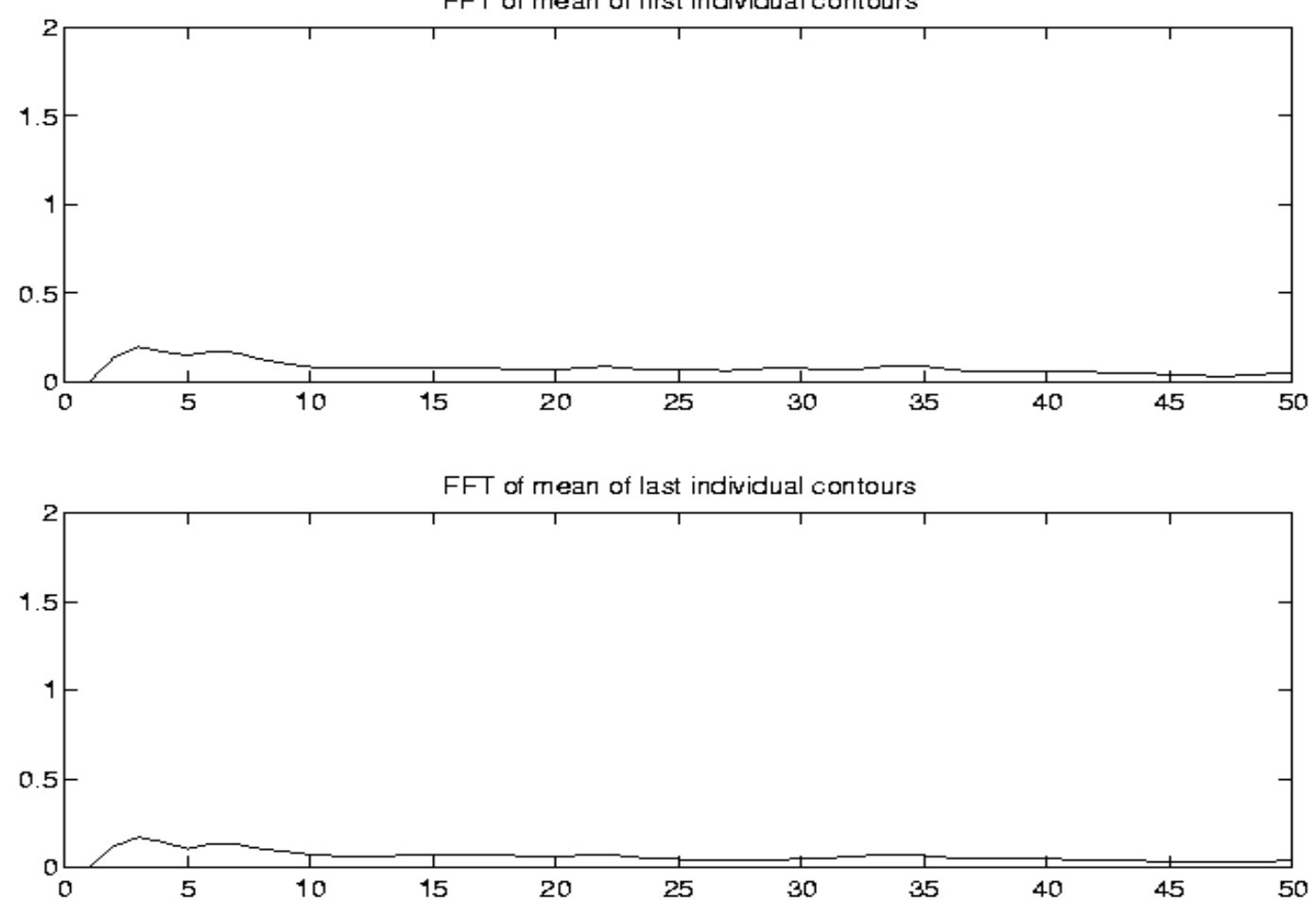

FFT for Static Measure 


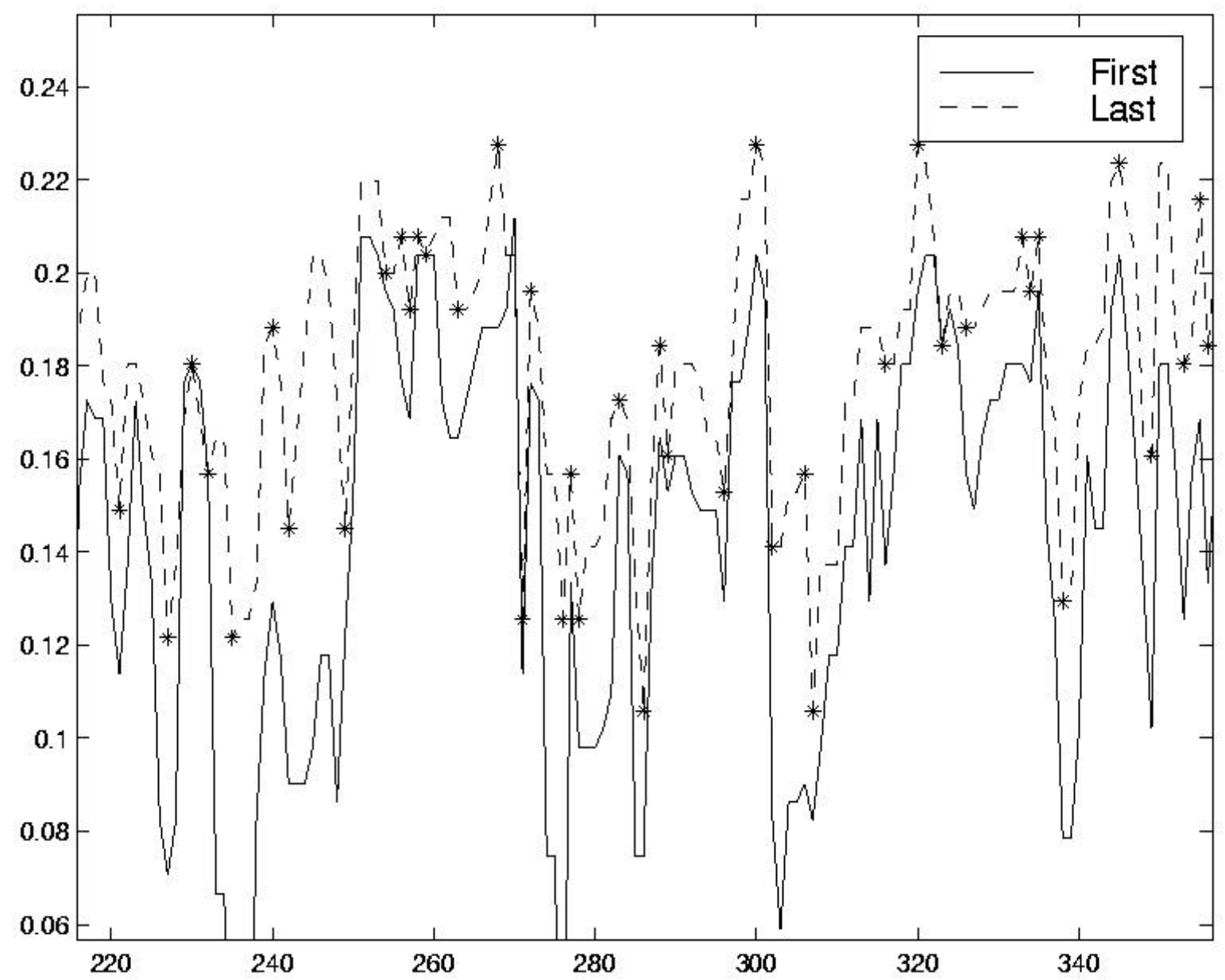

First versus last capture fingerprint signal, for dynamic measures. Extremums denoted by * 


\section{Extracted Parameters}

First Mean $=0.2566$ Last Mean $=0.2557$ MeanPercentChange $=-$ 0.334

FirstFFTstd $=2.4909$ LastFFTstd $=2.5959$

FirstFFTstdLPF $=2.5048$ LastFFTstdLPF $=2.6172$

First std $=0.0550$ Last $s t d=0.0562$ stdPercentChange $=2.030$

Last-FirstDiffMean=-0.001 Last-FirstDiffStd=0.019

Original: SumOfFirstMINS=129.7098 SumOfFirstMAXS=102.7882

SumOfLastMINS $=128.1765$ SumOfLastMAXS $=103.5647$

Original NORMALIZED:SumOfFirstMINS=0.336909

SumOfFirstMAXS $=0.191056$

SumOfLastMINS $=0.332926$ SumOfLastMAXS $=0.192499$

Filtered: SumOfFirstMINS=116.4516 SumOfFirstMAXS=129.7121

SumOfLastMINS=115.4057 SumOfLastMAXS=129.8163

Filtered NORMALIZED: SumOfFirstMINS $=0.243623$

SomeOfFirstMAXS $=0.271364$

SumOfLastMINS=0.241435 SumOfLastMAXS=0.271582

Original: FirstToLastMAXSgrowth $=0.7765$

FirstToLastMINSgrowth $=-1.5333$

FirstMINStoMAXS sum $=-26.9216$ LastMINStoMAXSsum $=-24.6118$

Original NORMALIZED: FirstToLastMAXSgrowth=0.001443

FirstToLastMINSgrowth $=-0.003983$

FirstMINStoMAXSsum $=-0.050040$ LastMINStoMAXSsum $=-0.045747$

Filtered: FirstToLastMAXSgrowth $=0.1043$

FirstToLastMINSgrowth $=-1.0459$

FirstMINStoMAXSsum=13.2605 LastMINStoMAXSsum=14.4106

Filtered NORMALIZED: FirstToLastMAXSgrowth=0.001624

FirstToLastMINSgrowth $=-0.003208$

FirstMINStoMAXSsum $=-0.056321$ LastMINStoMAXSsum $=-0.051489$

Original NORMALIZED: FirstTotalSwing=0.013794

Last Totalswing $=0.012888$

Filtered NORMALIZED: FilteredFirstTotalSwing=0.011433

FilteredLastTotalSwing $=0.010458$

stdFFTMeanFirst $=0.0388$ FFTstdMeanLast $=0.0374$ 
Appendix E: Flowchart of The Algorithm 


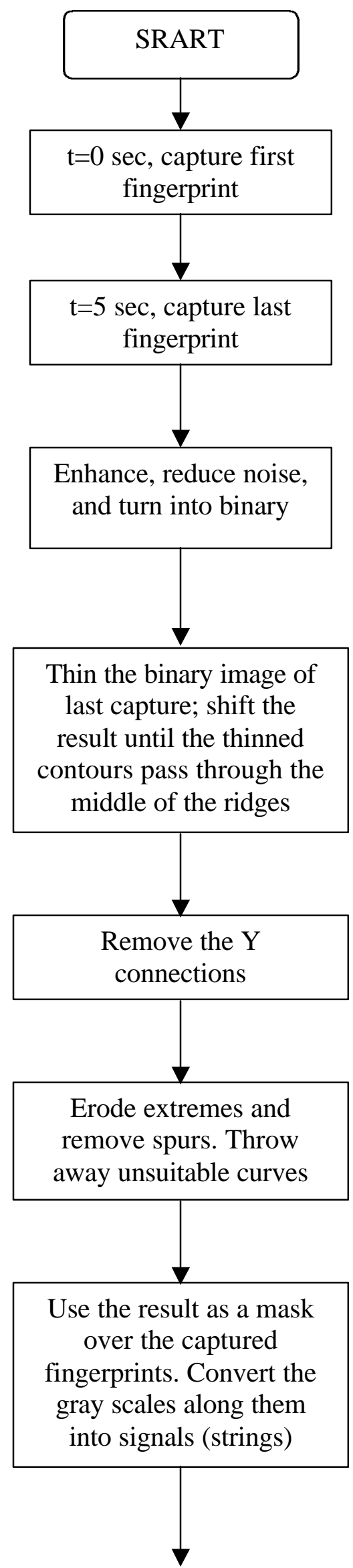




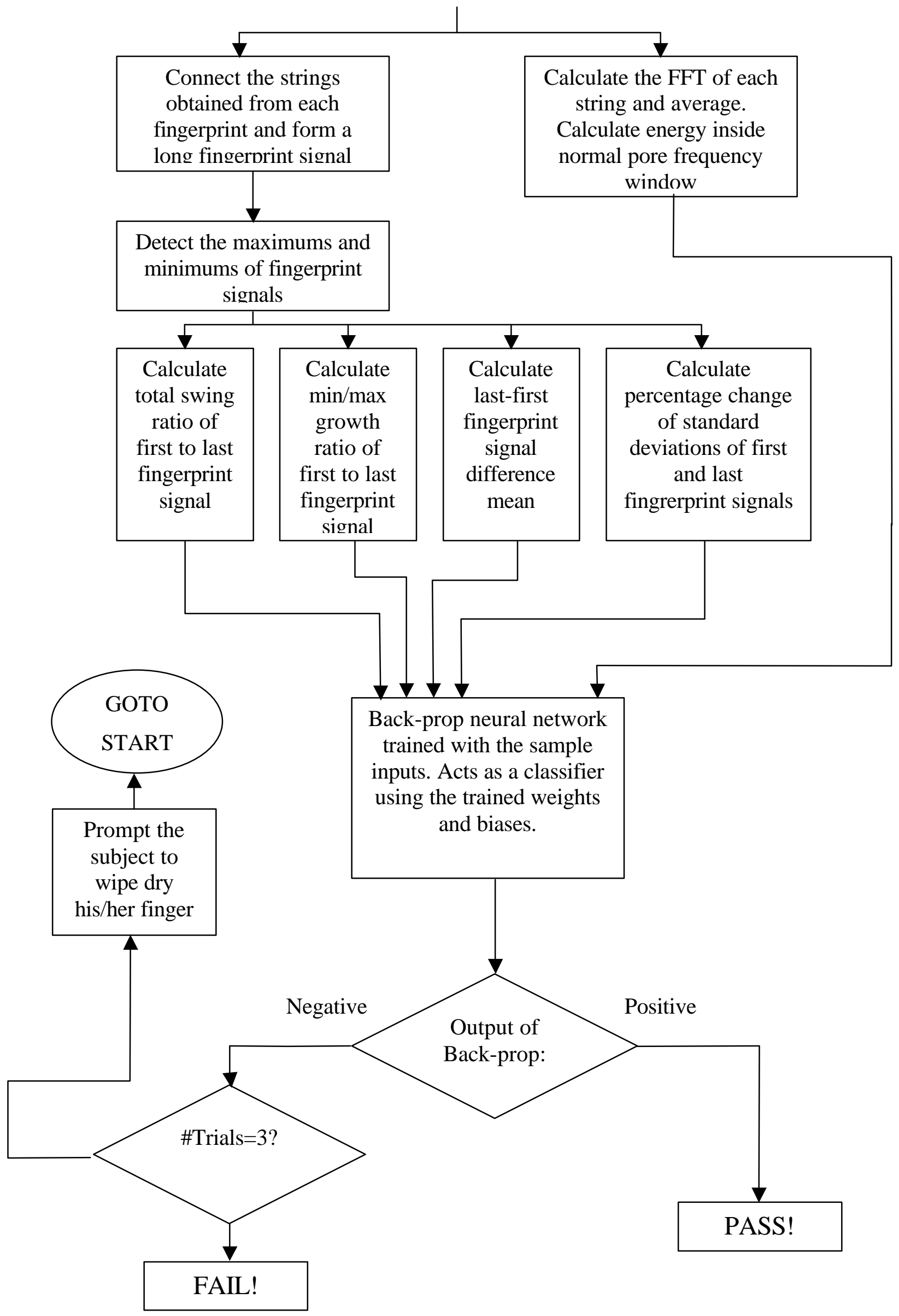

